\title{
Lectures on the functional renormalization group method
}

\author{
Janos Polonyi ${ }^{1,2}$ * \\ 1 Laboratory of Theoretical Physics, \\ Louis Pasteur University, Strasbourg, France \\ 2 Department of Atomic Physics, \\ L. Eötvös University, Budapest, Hungary
}

Received 1 July 2002; revised 1 August 2002

\begin{abstract}
These introductory notes are about functional renormalization group equations and some of their applications. It is emphasised that the applicability of this method extends well beyond critical systems, it actually provides us a general purpose algorithm to solve strongly coupled quantum field theories. The renormalization group equation of F. Wegner and A. Houghton is shown to resum the loop-expansion. Another version, due to J. Polchinski, is obtained by the method of collective coordinates and can be used for the resummation of the perturbation series. The genuinely non-perturbative evolution equation is obtained by a manner reminiscent of the Schwinger-Dyson equations. Two variants of this scheme are presented where the scale which determines the order of the successive elimination of the modes is extracted from external and internal spaces. The renormalization of composite operators is discussed briefly as an alternative way to arrive at the renormalization group equation. The scaling laws and fixed points are considered from local and global points of view. Instability induced renormalization and new scaling laws are shown to occur in the symmetry broken phase of the scalar theory. The flattening of the effective potential of a compact variable is demonstrated in case of the sine-Gordon model. Finally, a manifestly gauge invariant evolution equation is given for QED.

(C) Central European Science Journals. All rights reserved.
\end{abstract}

Keywords: strongly coupled theories, non-perturbative approximation, universality PACS (2000): 25.75.-q

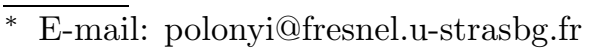




\section{Contents}

1 Introduction

2 Functional RG equations

2.1 Resumming the loop expansion

2.1.1 Blocking in continuous space-time

2.1.2 Local potential approximation

$\begin{array}{ll}2.1 .3 & \text { Gradient expansion }\end{array}$

2.2 Resumming the perturbation expansion 14

$\begin{array}{lll}2.2 .1 & \text { Polchinski equation } & 15\end{array}$

$\begin{array}{ll}2.2 .2 \text { Gradient expansion } & 18\end{array}$

$\begin{array}{lll}2.3 & \text { Composite operator renormalization } & 19\end{array}$

$\begin{array}{lll}2.3 .1 & \text { Toy model } & 20\end{array}$

2.3.2 Quantum Field Theory 21

2.3.3 Parallel transport 23

2.3.4 Asymptotical scaling 23

2.3.5 Perturbative treatment 25

2.4 Continuous evolution 25

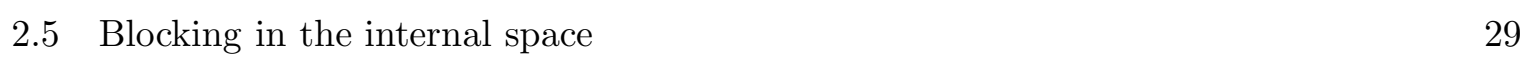

$\begin{array}{lll}2.5 .1 & \text { Wilsonian action } & 30\end{array}$

$\begin{array}{lll}2.5 .2 & \text { Effective action } & 31\end{array}$

3 Applications 33

3.1 Fixed points 34

3.1.1 Rescaling 34

$\begin{array}{lll}3.1 .2 & \text { Reparametrizing } & 35\end{array}$

$\begin{array}{lll}3.1 .3 & \text { Local potential approximation } & 36\end{array}$

3.1.4 Anomalous dimension 37

$\begin{array}{lll}3.2 & \text { Global RG } & 38\end{array}$

3.2.1 RG in Statistical and High Energy Physics $\quad 38$

$\begin{array}{lll}3.2 .2 & \text { Scalar model } & 42\end{array}$

3.2.3 RG microscope 46

3.2.4 The Theory of Everything 50

3.3 Instability induced renormalization 50

$\begin{array}{ll}\text { 3.3.1 Unstable effective potential } & 52\end{array}$

$\begin{array}{lll}3.3 .2 & \text { Tree-level WH equation } & 53\end{array}$

$\begin{array}{lll}3.3 .3 & \text { Plane wave saddle points } & 53\end{array}$

$\begin{array}{lll}3.3 .4 & \text { Correlation functions } & 55\end{array}$

$\begin{array}{ll}3.3 .5 & \text { Condensation as crossover } \\ \end{array}$

3.4 Sine-Gordon model $\quad 57$

$\begin{array}{lll}\text { 3.4.1 Zoology of the sine-Gordon model } & 58\end{array}$

$\begin{array}{lll}3.4 .2 & \text { Effective potential } & 59\end{array}$

$\begin{array}{lll}\text { 3.4.3 Breakdown of the fundamental group symmetry } & 61\end{array}$

3.4.4 Lower critical dimension $\quad 62$

3.5 Gauge models $\quad 62$

$\begin{array}{lll}\text { 3.5.1 Evolution equation } & 63\end{array}$

3.5.2 Gauge invariance 64

4 What has been achieved $\quad 65$

$\begin{array}{ll}\text { References } & 66\end{array}$ 


\section{Introduction}

The origin of renormalization goes back to hydrodynamics, the definition of the mass and other dynamical characteristics of bodies immersed into fluids. The more systematic elaboration of this concept is based on the (semi) group property of changing the observational scale in a theory. The renormalization group (RG) method has already been used with a number of different goals. Some of the more important directions are (i) to remove U.V. divergences [1], (ii) to describe the scale dependence of physical parameters and to classify the parameters of a theory around a critical point according to their impact on the dynamics [2], (iii) to express the highly singular product of local field variables [3], (iv) to resum the perturbation expansion in Quantum Field Theory [4] and in the case of differential equations [5] and finally (v) to solve strongly coupled theories. Furthermore, the RG method offers a systematic way of organizing our understanding of a complicated dynamics by successively eliminating degrees of freedom. The distinctive feature of the method is that it retains the influence of the eliminated subsystem on the rest.

The main subject of these lectures is application $(\mathrm{v})$ in the framework of the functional formalism, the search of a general purpose algorithm to handle non-perturbative and not necessarily critical theories.

The strategy of the RG scheme for the resummation of the perturbation expansion is to introduce and to evolve effective vertices instead of dealing with higher order corrections. The result is an iterative procedure where the contributions to the effective vertices computed at a given step in the leading order of the perturbation expansion and inserted in a graph at the next step reproduce the higher order corrections. In the traditional implementation of the RG procedure one follows the evolution of few coupling constants only. Our concern will be an improvement on this method in order to follow the evolution of a large number of coupling constants. This is realized by means of the functional formalism where the evolution is constructed for the generator function for all coupling constants.

Before starting let us review the conventional multiplicative RG schemes as used in Quantum Field Theory. This scheme is based on the relation

$$
G_{R}^{(n)}\left(p_{1}, \cdots, p_{n} ; g_{R}, \mu\right)=Z^{-n / 2} G_{B}^{(n)}\left(p_{1}, \cdots, p_{n} ; g_{B}, \Lambda\right)+\mathcal{O}\left(\frac{\mu^{2}}{\Lambda^{2}}\right)+\mathcal{O}\left(\frac{p_{j}^{2}}{\Lambda^{2}}\right)
$$

for the Green functions, where $\Lambda$ and $\mu$ denote the U.V. cut-off and the substraction (observational) scales, respectively. $Z$ depends on either $g_{R}$ or $g_{B}$ and the ratio $\Lambda / \mu$ (the mass is treated as one of the parameters $g$ ). The ignored terms stand for non-universal, cut-off dependent interactions.

Bare RG equation: The renormalized theory is independent of the choice of the cut-off,

$$
\Lambda \frac{d}{d \Lambda} G_{R}^{(n)}\left(p_{1}, \cdots, p_{n} ; g_{B}, \Lambda\right)=\Lambda \frac{d}{d \Lambda}\left[Z^{n / 2}\left(g_{B}, \Lambda\right) G_{B}^{(n)}\left(p_{1}, \cdots, p_{n} ; g_{B}, \Lambda\right)\right]=0,
$$

where the renormalized coupling constants and the substraction scale are kept fixed in computing the derivatives. The equation involving the renormalized quantities is not 
too useful. It is the multiplicative renormalization scheme which leads to the second equation, expressing the possibility of compensating the change of the substraction scale by the modification of the coupling constants $g_{B}(\Lambda)$ arising from the solution of the differential equation by the method of characteristics. Notice that the compensation is possible only if the list of the coupling constants $\left\{g_{B}\right\}$ includes all relevant coupling constants of the appropriate scaling regime, around the U.V. fixed point.

Renormalized RG equation: The bare theory is independent of the choice of the substraction scale,

$$
\mu \frac{d}{d \mu} G_{B}^{(n)}\left(p_{1}, \cdots, p_{n} ; g_{R}, \mu\right)=\mu \frac{d}{d \mu}\left[Z^{-n / 2}\left(g_{R}, \mu\right) G_{R}^{(n)}\left(p_{1}, \cdots, p_{n} ; g_{R}, \mu\right)\right]=0,
$$

where the bare coupling constants and the cut-off are kept fixed.

Callan-Symanzik equation: The change of the mass in the propagator is governed by the expression

$$
\frac{d}{d m^{2}} \frac{1}{p^{2}-m^{2}}=\frac{1}{p^{2}-m^{2}} \cdot \frac{1}{p^{2}-m^{2}}
$$

which can be used to find out the dependence on the renormalized mass when the cut-off and all bare parameters except the bare mass are kept fixed. The resulting evolution equation [6] is similar to the two previous RG equations except that the derivative is with respect to the bare mass instead of the cut-off or the substraction scale and the right hand side is non-vanishing. This latter feature indicates that contrary to the first two schemes the evolution $g_{R}\left(\mathrm{~m}^{2}\right)$ in the Callan-Symanzik equation is not a renormalized trajectory, it connects theories with different mass.

The serious limitation of these equations is that they are asymptotic, i.e., are applicable in the regime $\mu^{2}, p^{2}<<\Lambda^{2}$ only. In fact, the omission of the non-universal terms in the multiplicative renormalization scheme (1) requires that we stay away from the cut-off. In models with IR instability (spontaneous symmetry breaking, dynamical mass generation,...) another limitation arises, $m_{d y n}^{2}<<\mu^{2}, p^{2}$, to ensure that we stay in the U.V. scaling regime. This is because the IR scaling regime may have relevant operators which are irrelevant on the U.V. side [7]. In more realistic models with several scaling regimes which have different relevant operator sets we need non-asymptotic methods which can interpolate between the different scaling regimes. This is achieved by the functional extensions of the RG equation based on the infinitesimal blocking steps.

\section{Functional RG equations}

There are different avenues to arrive at a functional RG equation. The simplest is to follow Wilson-Kadanoff blocking in continuous space-time. It leads to a functional differential equation describing the cut-off dependence of the bare action. Better approximation schemes can be worked out when the modes are suppressed smoothly as the cut-off changes. The parameters of the bare action which are followed by these RG equations are related to observables qualitatively only. It is more advantageous to transform the RG equation for the effective action whose parameters have direct relation with observables. 
For the sake of simplicity we consider an Euclidean theory in $d$ dimensions for a scalar field $\phi_{x}$ governed by the action $S_{B}[\phi]$. An $O(d)$ invariant U.V. cut-off $\Lambda$ is introduced in the momentum space by requiring $\phi_{p}=0$ for $|p|>\Lambda$ to render the generator functional

$$
e^{\frac{1}{\hbar} W[j]}=\int D[\phi] e^{-\frac{1}{\hbar} S_{B}[\phi]+\frac{1}{\hbar} j \cdot \phi}
$$

finite. We shall use the following notation conventions, stated below: In order to render the functional manipulations well defined we always assume discrete spectrum, i.e., the presence of U.V. and IR regulators, say a lattice spacing $a$ and system volume $V=a^{d} N^{d}$. The space-time integrals are $\int d^{d} x=a^{d} \sum_{x}=\int_{x}, \int_{x} f_{x} g_{x}=f \cdot g, \int d^{d} p /(2 \pi)^{d}=V^{-1} \sum_{p}=$ $\int_{p}, f_{p}=\int_{x} e^{-i p x} f_{x}$, and $f_{x}=\int_{p} e^{i p x} f_{p}$. The Dirac-deltas $\delta_{x, y}=a^{-d} \delta_{x, y}^{K}$ and $\delta_{p, q}=V \delta_{p, q}^{K}$ are expressed in terms of the Kronecker-deltas $\delta_{x, y}^{K}$ and $\delta_{p, q}^{K}$.

\subsection{Resumming the loop expansion}

We start with the simplest form of infinitesimal blocking-step RG equations which is the functional extension of the bare RG scheme mentioned above [8].

\subsubsection{Blocking in continuous space-time}

We shall denote the moving U.V. cut-off by $k$. Its lowering $k \rightarrow k-\Delta k$ leads to the blocking transformation of the action which preserves the generator functional (5). Due to the presence of the source this blocking introduces an explicit source dependence in the action,

$$
\int D_{k}[\phi] e^{-\frac{1}{\hbar} S_{k}[\phi ; j]+\frac{1}{\hbar} j \cdot \phi}=\int D_{k-\Delta k}[\phi] e^{-\frac{1}{\hbar} S_{k-d k}[\phi ; j]+\frac{1}{\hbar} j \cdot \phi},
$$

where $D_{k}[\phi]$ stands for the integration measure over the functional space $\mathcal{F}_{k}$ consisting of functions whose Fourier transform is non-vanishing for $|p| \leq k$. In order to avoid this complication one usually assumes $j \in \mathcal{F}_{k-\Delta k}$. In this case the blocking becomes a mapping $S_{k}[\phi] \rightarrow S_{k-\Delta k}[\phi]$ and it is enough impose the invariance of the partition function

$$
e^{-\frac{1}{\hbar} S_{k-\Delta k}[\phi]}=\int D[\tilde{\phi}] e^{-\frac{1}{\hbar} S_{k}[\phi+\tilde{\phi}]},
$$

where $\phi \in \mathcal{F}_{k-\Delta k}$ and $\tilde{\phi} \in \mathcal{F}_{k} \backslash \mathcal{F}_{k-\Delta k}$.

The evaluation of the path integral by means of the loop-expansion gives immediately the functional RG equation,

$$
S_{k-\Delta k}[\phi]=S_{k}\left[\phi+\tilde{\phi}_{c l}\right]+\frac{\hbar}{2} \operatorname{tr} \ln \frac{\delta^{2} S_{k}\left[\phi+\tilde{\phi}_{c l}\right]}{\delta \tilde{\phi} \delta \tilde{\phi}}+\mathcal{O}\left(\hbar^{2}\right),
$$

where $\tilde{\phi}_{c l}$ denotes the saddle point. The trace is over the functional space $\tilde{\phi} \in \mathcal{F}_{k} \backslash \mathcal{F}_{k-\Delta k}$ and $\mathcal{O}\left(\hbar^{2}\right)$ represents the higher loop contributions. We write this equation as

$$
S_{k}[\phi]-S_{k-\Delta k}[\phi]=\underbrace{S_{k}[\phi]-S_{k}\left[\phi+\tilde{\phi}_{c l}\right]}_{\text {tree level }}-\underbrace{\frac{\hbar}{2} \operatorname{tr} \ln \frac{\delta^{2} S_{k}\left[\phi+\tilde{\phi}_{c l}\right]}{\delta \tilde{\phi} \delta \tilde{\phi}}+\mathcal{O}\left(\hbar^{2}\right)}_{\text {loop contributions }} .
$$



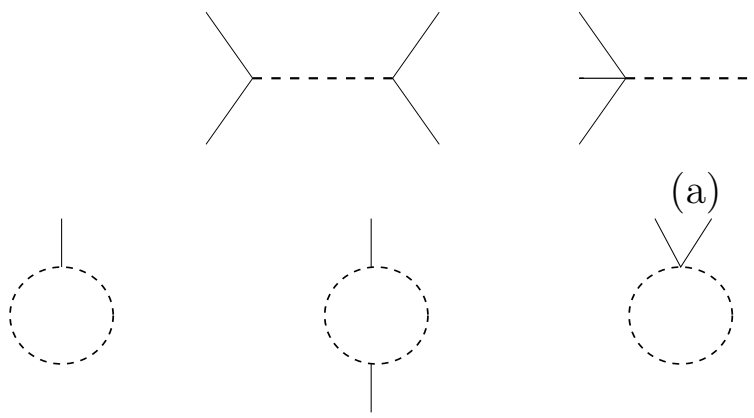

(b)
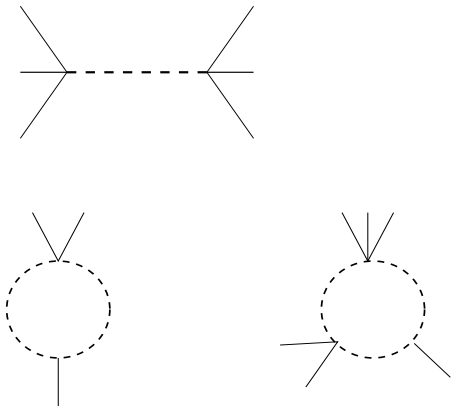

Fig. 1 Graphs contributing to the blocking, (a): tree-level, (b): one-loop corrections. The dashed line stands for a particle of momentum $|p|=k$ and the solid lines represent $\phi$.

When can one safely assume that the saddle point is trivial, $\tilde{\phi}_{c l}=0$ ? Let us suppose that $\phi_{x}$ is weakly and slowly varying, ie $\phi_{x}=\Phi+\eta_{x}$ where $\eta_{x} \approx 0$ and the characteristic momentum of $\eta$ is small with respect to the cut-off $k$. Then the fluctuating component $\eta$ appears as an external source breaking translation invariance. The saddle point, $\tilde{\phi} \in$ $\mathcal{F}_{k} \backslash \mathcal{F}_{k-\Delta k}$, being inhomogeneous exists as $\eta \rightarrow 0$ only if the external space-time symmetry is broken dynamically.

But a $\Delta k$-dependent small saddle point, $\tilde{\phi}_{\mathrm{cl}}=\mathcal{O}\left(\Delta k^{n}\right), n>0$ may occur without breaking external symmetries. In this case we expand in $\tilde{\phi}_{\mathrm{cl}}$ and find the WegnerHoughton (WH) equation [8]

$$
\begin{aligned}
S_{k}[\phi]-S_{k-\Delta k}[\phi]= & \frac{1}{2} \frac{\delta S_{k}[\phi]}{\delta \tilde{\phi}} \cdot\left(\frac{\delta^{2} S_{k}[\phi]}{\delta \tilde{\phi} \delta \tilde{\phi}}\right)^{-1} \cdot \frac{\delta S_{k}[\phi]}{\delta \tilde{\phi}} \\
& -\frac{\hbar}{2} \operatorname{tr} \ln \frac{\delta^{2} S_{k}[\phi]}{\delta \tilde{\phi} \delta \tilde{\phi}}+\mathcal{O}\left(\hbar^{2}\right)+\mathcal{O}\left(\Delta k^{2}\right)
\end{aligned}
$$

where all functional derivatives are taken at $\tilde{\phi}=0$. The saddle point can be omitted in the argument of the logarithmic function because the trace brings a factor of $\Delta k$ as we shall see below. The discussion of the tree-level renormalization when $\tilde{\phi}_{\mathrm{cl}}$ is non-vanishing and finite as $\Delta k \rightarrow 0$ is deferred to section 3.3.

One can understand the loop contribution better by splitting the action into the sum of the quadratic part and the rest, $S=\frac{1}{2} \phi \cdot G_{0}^{-1} \cdot \phi+S_{i}$ and expanding in $S_{i}$,

$$
\operatorname{tr} \ln \frac{\delta^{2} S_{k}[\phi]}{\delta \tilde{\phi} \delta \tilde{\phi}}=\operatorname{tr} \ln G_{0}^{-1}-\sum_{n=1}^{\infty} \frac{(-1)^{n}}{n} \operatorname{tr}\left(\frac{\delta^{2} S_{k}[\phi]}{\delta \tilde{\phi} \delta \tilde{\phi}} \cdot G_{0}\right)^{n} .
$$

We recovered the sum of one-loop graphs. The loop corrections close on all possible pair of $\tilde{\phi}$ legs with the propagator $G_{0}$. The tree-level piece describes the feedback of the change of the cut-off on the dynamics of the classical background field $\phi$. This is classical contribution because the cut-off controls the configuration space, the number of degrees of freedom. Some of the graphs contributing to the right hand side are shown in Fig. 1.

The point of central importance is that $\Delta k$ serves here as a new small parameter to suppress the higher-loop contributions. As an example consider the simplest case, 
$\phi_{x}=\Phi, \tilde{\phi}_{c l}=0$ with the ansatz

$$
S_{k}[\phi]=\int_{x}\left[\frac{1}{2} Z_{k}\left(\phi_{x}\right)\left(\partial_{\mu} \phi_{x}\right)^{2}+U_{k}\left(\phi_{x}\right)\right]
$$

for the action with $Z(\phi)=1$,

$$
U_{k-\Delta k}(\Phi)=U_{k}(\Phi)+\frac{\hbar}{2} \int_{k-\Delta k<|p|<k} \ln \left[p^{2}+U_{k}^{\prime \prime}(\Phi)\right]+\mathcal{O}\left((\hbar \Delta k)^{2}\right) .
$$

Each loop-integral is over the shell $k-\Delta k<|p|<k$ in momentum space. So long the propagator is non-singular in the integration domain the n-loop integrals will be proportional to the $n$-th power of the integration volume, giving a dimensionless small suppression parameter $\approx(\Delta k / k)^{n}$, the proportion of the modes eliminated with those left. The question of the singularity will be considered in section 3.2.2. The higher loop contributions are suppressed in the infinitesimal blocking step limit and the one-loop evolution equation turns out to be an exact functional equation. The limit $\Delta k \rightarrow 0$ is safe for the loop contributions but more care is needed when the saddle point is non-trivial because the tree-level contribution might be singular in this limit.

Notice that the convergence of the loop expansion was assumed in the argument above. Another word of caution is in order about Eq. (9). Not only the trace but the logarithm function itself is considered within the subspace $\tilde{\phi} \in \mathcal{F}_{k} \backslash \mathcal{F}_{k-\Delta k}$, a rather formal observation which becomes essential later.

It is sometimes easy to arrive at exact, but unmanageable equations. The problem is rather to find an approximation, an acceptable compromise between precision and simplicity. By assuming the absence of non-local interactions and the homogeneity of the vacuum the blocked action is usually expanded in the gradient of the field, leading to the ansatz (12) for the action where contributions $\mathcal{O}\left(\partial^{4}\right)$ are neglected. This step is the key to the success of the RG method. The point is that the blocked action can be identified by evaluating the integral (7) for different background configurations. The determination of the blocked action is an over-constrained problem because there are 'more' background field configurations than coupling constants in a given ansatz. The tacit assumption, that the effective action is well defined, i.e., independent of the set of configurations used to read off its coupling constants, gives the real power of the $R G$ method: it enables us to make predictions.

\subsubsection{Local potential approximation}

In order to relate the WH equation to conventional perturbation expansion we shall use the ansatz (12) with $Z=1$, in the local potential approximation. In order to determine the only non-trivial piece of this action the potential $U_{k}(\phi)$ it is sufficient to consider a homogeneous field $\phi(x)=\Phi$ in (9). The saddle point is vanishing as mentioned above, $\tilde{\phi}_{c l}=0$, so long as the external space-time symmetries are unbroken. The blocking equation simplified in this manner yields Eq. (13), the projection of the WH equation into the restricted space of actions,

$$
\dot{U}_{k}(\Phi)=-\frac{\hbar \Omega_{d} k^{d}}{2(2 \pi)^{d}} \ln \left[k^{2}+U_{k}^{(2)}(\Phi)\right]
$$



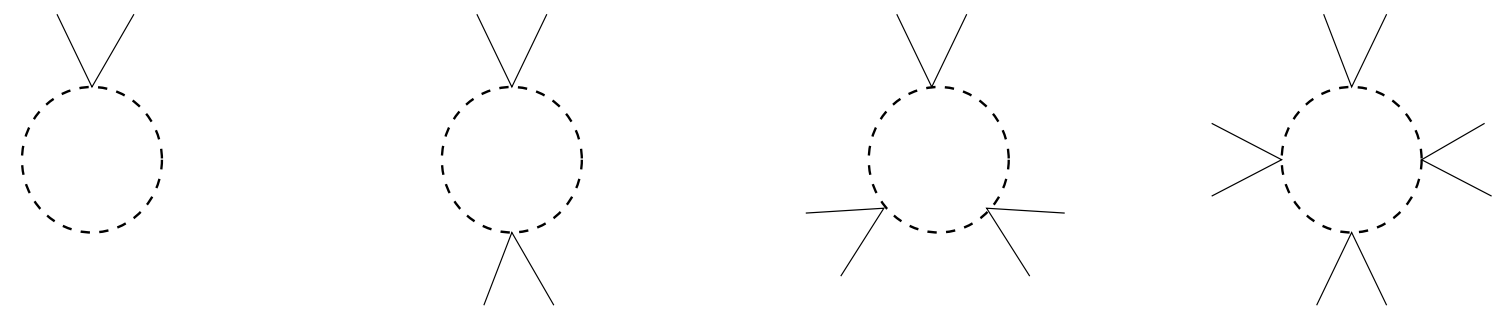

Fig. 2 The first four one-loop graphs contributing to the WH equation in the local potential approximation when the potential $U(\phi)$ is truncated to the terms $\phi^{2}$ and $\phi^{4}$. The dashed line corresponds a particle of momentum $|p|=k$.

where the dot stands for $k \partial_{k}$ and $\Omega_{d}=2 \pi^{d / 2} / \Gamma(d / 2)$ denotes the solid angle.

The expansion of the logarithmic function leads to the series

$$
\dot{U}_{k}(\Phi)=-\frac{\hbar \Omega_{d} k^{d}}{2(2 \pi)^{d}}\left\{\ln \left[k^{2}+m^{2}\right]-\sum_{n=1}^{\infty} \frac{(-1)^{n}}{n}\left(\frac{U_{k}^{(2)}(\Phi)-m^{2}}{k^{2}+m^{2}}\right)^{n}\right\},
$$

where $m^{2}=U^{(2)}(0)$, the sum of the one-loop graphs whose external legs have vanishing momentum and the internal lines carry momentum $|p|=k$, cf Fig. 2, the leading order contributions to the renormalization of the coupling constants $g_{n}$ in the loop expansion.

The partial resummation of the perturbation series performed by the WH equation can easily be seen by comparing the solution of Eq. (14) with those obtained in the independent mode approximation, where the $k$-dependence is ignored in the right hand side of the equation,

$$
U_{k}(\Phi)=U_{\lambda}(\Phi)-\frac{\hbar}{2} \int_{k<|p|<\Lambda} \ln \left[p^{2}+U_{\Lambda}^{(2)}(\Phi)\right]
$$

One recovers the one-loop effective potential at the IR fixed point, $V_{\text {eff }}(\Phi)=U_{k=0}(\Phi)$. This is not by accident and remains valid for the complete solution, as well, because $e^{-V V_{\text {eff }}(\Phi)}$ is the distribution of the homogeneous mode and the blocking (7) at $k=0$ retains the homogeneous mode only, $S_{k=0}[\Phi]=V U_{k=0}(\Phi)$. We shall see in section 3.3 that this simple argument is valid in the absence of large amplitude inhomogeneous instabilities only.

Let us consider as an example the differential equation

$$
\dot{x}_{k}=f\left(x_{k}\right), \quad x_{\Lambda}=x_{B},
$$

where $f(x)$ is weakly varying analytic function given in its expanded form around the base point $x=0$,

$$
\dot{x}_{k}=\sum_{n} \frac{f^{(n)}(0)}{n !} x_{k}^{n}
$$

The RG method allows us to perform the expansion at the current point,

$$
x_{k-\Delta k}=x_{k}-\Delta k f\left(x_{k}\right)+\mathcal{O}\left(\Delta k^{2}\right),
$$


cf Eq. (8). The $k$-dependence of the right hand side represents the accumulation of the information obtained during the integration. In a similar manner, the virtue of the functional RG scheme (9) is the expansion of the path integral around around the current action, instead of the Gaussian fixed point.

A more detailed picture of the $\mathrm{RG}$ flow can be obtained by inspecting the beta functions. For this end we parametrize the potential as

$$
U_{k}(\phi)=\sum_{n} \frac{g_{n}(k)}{n !}\left(\phi-\phi_{0}\right)^{n}
$$

and write the beta functions corresponding to $\phi_{0}=\langle\phi\rangle$ as

$$
\beta_{n}=k \partial_{k} g_{n}(k)=k \partial_{k} \partial_{\phi}^{n} U_{k}\left(\phi_{0}\right)=\partial_{\phi}^{n} k \partial_{k} U_{k}\left(\phi_{0}\right)=-\frac{\hbar \Omega_{d} k^{d}}{2(2 \pi)^{d}} \partial_{\phi}^{n} \ln \left[k^{2}+\partial_{\phi}^{2} U_{k}(\phi)\right]
$$

It is easy to see that $\beta_{n}$ is an $n$-th order polynomial

$$
\beta_{n}=-\frac{\hbar \Omega_{d} k^{d}}{2(2 \pi)^{d}} \mathcal{P}_{n}\left[G_{3}, \cdots, G_{n+2}\right]
$$

of the expression $G_{n}=g_{n} /\left(k^{2}+g_{2}\right)$, eg

$$
\begin{aligned}
\mathcal{P}_{2}= & G_{4}-G_{3}^{2}, \\
\mathcal{P}_{3}= & G_{5}-3 G_{3} G_{4}+2 G_{3}^{3}, \\
\mathcal{P}_{4}= & G_{6}-4 G_{3} G_{5}+12 G_{3}^{2} G_{4}-3 G_{4}^{2}-6 G_{3}^{4}, \\
\mathcal{P}_{5}= & G_{7}-5 G_{3} G_{6}+20 G_{3}^{2} G_{5}-10 G_{4} G_{5}-60 G_{3}^{3} G_{4}+30 G_{3} G_{4}^{2}+24 G_{3}^{5}, \\
\mathcal{P}_{6}= & G_{8}-6 G_{3} G_{7}+30 G_{3}^{2} G_{6}-5 G_{5} G_{6}-120 G_{3}^{3} G_{5}+120 G_{3} G_{4} G_{5} \\
& -270 G_{3}^{2} G_{4}^{2}-10 G_{5}^{2}-10 G_{4} G_{6}+360 G_{3}^{4} G_{4}+30 G_{4}^{3}-120 G_{3}^{6},
\end{aligned}
$$

etc. The correspondence between these expressions and Feynman graphs contributing to the beta functions in the traditional renormalization scheme is indicated in Fig. 3.

We shall need later the beta functions corresponding to coupling constants made dimensionless by the running cut-off. By means of the parametrization $\phi=k^{(d-2) / 2} \tilde{\phi}$, $g_{n}=k^{\left[g_{n}\right]} \tilde{g}_{n}$,

$$
\left[g_{n}\right]=d+n\left(1-\frac{d}{2}\right)
$$

$U(\phi)=k^{d} \tilde{U}(\tilde{\phi})$ one finds

$$
\tilde{\beta}_{n}(\tilde{\phi})=\dot{\tilde{g}}_{n}(k)=k^{-\left[g_{n}\right]} \beta_{n}(\phi)-\left[g_{n}\right] \tilde{g}_{n}=-\frac{\hbar \Omega_{d}}{2(2 \pi)^{d}} \mathcal{P}_{n}\left[\tilde{G}_{3}, \cdots, \tilde{G}_{n+2}\right]-\left[g_{n}\right] \tilde{g}_{n},
$$

where $\tilde{G}_{n}=\tilde{g}_{n} /\left(1+\tilde{g}_{2}\right)$.

An instructive way to read Eqs. (20) and (14) is that the potential $U(\phi)$ is the generator function for the coupling constants. The functional RG method is an economical book-keeping method for the computation of graphs and their symmetry factors by keeping track of their generator function(al). 

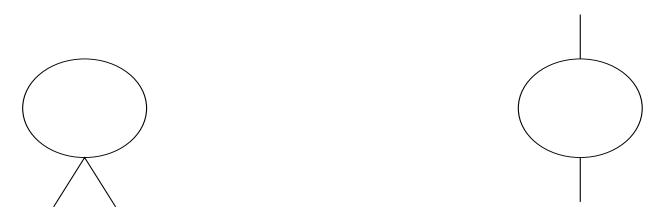

(a)
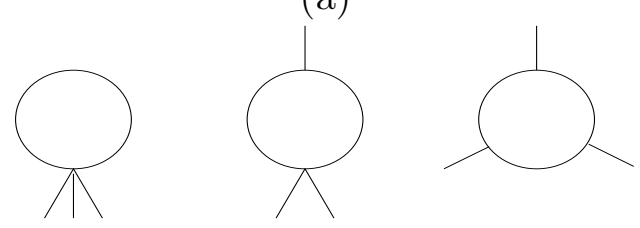

(b)
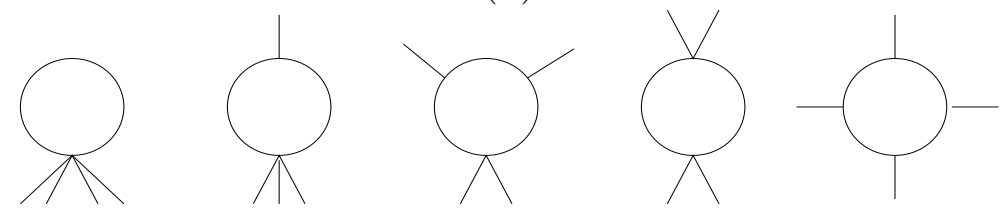

(c)

Fig. 3 Graphs contributing to $\beta_{n}$, (a): $n=2,(\mathrm{~b}): n=3$ and (c): $n=4$.

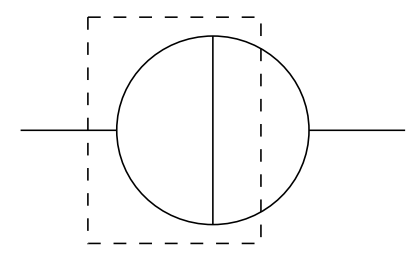

Fig. 4 A two-loop contribution to $g_{2}$.

The higher loop contributions to the running coupling constants are generated by the integration of the differential equations $k \partial_{k} g_{n}=\beta_{n}$. The us consider the two-loop graph depicted in Fig. 4 and expand the subgraph in the square in the momenta of its external legs. The zeroth order contributions is the last graph of Fig. 3b. By going higher order in the gradient expansion one can, in principle, generate the full momentum dependence of the subgraph. In general, the $n$-th loop contributions appear in the integration after $n$ step $k \rightarrow k+\Delta k$ and all loops are resummed in the limit $\Delta k \rightarrow 0$.

\subsubsection{Gradient expansion}

The natural expansion for the long distance properties of systems with homogeneous ground state is the expansion in the inverse characteristic length of the fluctuations, in the gradient operator acting on the field variable.

Functional derivatives of local functionals: We consider the following generalization of the $\mathcal{O}\left(\partial^{2}\right)$ ansatz $(12)$,

$$
S[\phi]=\int_{x}\left[\frac{1}{2} Z\left(\phi_{x}\right) \partial_{\mu} \phi_{x} K^{-1}(-\square) \partial_{\mu} \phi_{x}+U\left(\phi_{x}\right)\right] .
$$

We shall use the notation $Z_{x}=Z\left(\phi_{x}\right), U_{x}=U\left(\phi_{x}\right)$ for the coefficient functions and $Z_{x}^{(n)}=\partial_{\phi}^{n} Z\left(\phi_{x}\right), U_{x}^{(n)}=\partial_{\phi}^{n} U\left(\phi_{x}\right)$ for their derivatives. The first two functional derivatives 
of the action are

$$
\begin{aligned}
V \frac{\delta S}{\delta \phi_{p}}= & \sum_{y} e^{i p y} \frac{\delta S}{\delta \phi_{y}} \\
= & \int_{x, y} e^{i p y}\left[\delta_{x, y} U_{x}^{(1)}+\frac{1}{2} \delta_{x, y} Z_{x}^{(1)} \partial_{\mu} \phi_{x} K^{-1} \partial_{\mu} \phi_{x}\right. \\
& \left.+\frac{1}{2} Z_{x}\left(\partial_{\mu} \delta_{x, y} K^{-1} \partial_{\mu} \phi_{x}+\partial_{\mu} \phi_{x} K^{-1} \partial_{\mu} \delta_{x, y}\right)\right],
\end{aligned}
$$

and

$$
\begin{aligned}
V^{2} \frac{\delta^{2} S}{\delta \phi_{p} \delta \phi_{q}}= & \int_{x, y, z} e^{i p y+i q z}\left\{\frac{1}{2} \delta_{x, y} \delta_{x, z} Z_{x}^{(2)} \partial_{\mu} \phi_{x} K^{-1} \partial_{\mu} \phi_{x}\right. \\
& +\frac{1}{2} Z_{x}\left(\partial_{\mu} \delta_{x, y} K^{-1} \partial_{\mu} \delta_{x, z}+\partial_{\mu} \delta_{x, z} K^{-1} \partial_{\mu} \delta_{x, y}\right) \\
& +\frac{1}{2} Z_{x}^{(1)}\left[\delta_{x, y}\left(\partial_{\mu} \delta_{x, z} K^{-1} \partial_{\mu} \phi_{x}+\partial_{\mu} \phi_{x} K^{-1} \partial_{\mu} \delta_{x, z}\right)\right. \\
& \left.+\delta_{x, z}\left(\partial_{\mu} \delta_{x, y} K^{-1} \partial_{\mu} \phi_{x}+\partial_{\mu} \phi_{x} K^{-1} \partial_{\mu} \delta_{x, y}\right)\right] \\
& \left.+\delta_{x, y} \delta_{x, z} U_{x}^{(2)}\right\} .
\end{aligned}
$$

We split the field into a homogeneous background and fluctuations, $\phi_{x}=\Phi+\eta_{x}$ and find up to $\mathcal{O}\left(\eta^{2}\right)$,

$$
\begin{aligned}
V \frac{\delta S}{\delta \phi_{p}}= & \int_{x, y} e^{i p y}\left\{\delta_{x, y}\left(U^{(1)}+U^{(2)} \eta_{x}+\frac{1}{2} U^{(3)} \eta_{x}^{2}\right)+\frac{1}{2}\left[\delta_{x, y} Z^{(1)} \partial_{\mu} \eta_{x} K^{-1} \partial_{\mu} \eta_{x}\right.\right. \\
& \left.\left.+\left(Z+Z^{(1)} \eta_{x}\right)\left(\partial_{\mu} \delta_{x, y} K^{-1} \partial_{\mu} \eta_{x}+\partial_{\mu} \eta_{x} K^{-1} \partial_{\mu} \delta_{x, y}\right)\right]\right\} \\
= & \int_{x, y} e^{i p y} \delta_{x, y}\left\{\left(U^{(1)}+U^{(2)} \int_{r} e^{i r \cdot x} \eta_{r}+\frac{1}{2} U^{(3)} \int_{r, s} e^{i(r+s) \cdot x} \eta_{r} \eta_{s}\right)\right. \\
& -\frac{1}{2}\left[Z^{(1)} \int_{r, s} e^{i(r+s) \cdot x} r \cdot s \eta_{r} K_{s}^{-1} \eta_{s}\right. \\
& \left.\left.+\left(Z+Z^{(1)} \int_{r} e^{i r \cdot x} \eta_{r}\right) \int_{s} p \cdot s e^{i s \cdot x} \eta_{s}\left(K_{s}^{-1}+K_{p}^{-1}\right)\right]\right\} \\
= & \delta_{p, 0} U^{(1)}+U^{(2)} \eta_{-p}+\frac{1}{2} p^{2} Z\left(K_{-p}^{-1}+K_{p}^{-1}\right) \eta_{-p} \\
& +\frac{1}{2} \int_{r, s} \delta_{p+r+s, 0} \eta_{r} \eta_{s}\left[U^{(3)}-Z^{(1)}\left(r \cdot s K_{s}^{-1}+p \cdot r K_{r}^{-1}+p \cdot s K_{p}^{-1}\right)\right]
\end{aligned}
$$

and

$$
\begin{aligned}
V^{2} \frac{\delta^{2} S}{\delta \phi_{p} \delta \phi_{q}}= & \int_{x, y, z} e^{i p y+i q z}\left\{\delta_{x, y} \delta_{x, z}\left(U^{(2)}+U^{(3)} \eta_{x}+\frac{1}{2} U^{(4)} \eta_{x}^{2}\right)\right. \\
& +\frac{1}{2}\left[\delta_{x, y} \delta_{x, z} Z^{(2)} \partial_{\mu} \eta_{x} K^{-1} \partial_{\mu} \eta_{x}\right. \\
& +\delta_{x, y}\left(Z^{(1)}+Z^{(2)} \eta_{x}\right)\left(\partial_{\mu} \delta_{x, z} K^{-1} \partial_{\mu} \eta_{x}+\partial_{\mu} \eta_{x} K^{-1} \partial_{\mu} \delta_{x, z}\right)
\end{aligned}
$$




$$
\begin{aligned}
& +\delta_{x, z}\left(Z^{(1)}+Z^{(2)} \eta_{x}\right)\left(\partial_{\mu} \delta_{x, y} K^{-1} \partial_{\mu} \eta_{x}+\partial_{\mu} \eta_{x} K^{-1} \partial_{\mu} \delta_{x, y}\right) \\
& \left.\left.+\left(Z+Z^{(1)} \eta_{x}+\frac{1}{2} Z^{(2)} \eta_{x}^{2}\right)\left(\partial_{\mu} \delta_{x, z} K^{-1} \partial_{\mu} \delta_{x, y}+\partial_{\mu} \delta_{x, y} K^{-1} \partial_{\mu} \delta_{x, z}\right)\right]\right\} \\
= & \int_{x, y, z} e^{i p y+i q z} \delta_{x, y} \delta_{x, z}\left\{-\frac{1}{2}\left[Z^{(2)} \int_{r, s} r \cdot s e^{i r x} \eta_{r} K_{s}^{-1} e^{i s x} \eta_{s}\right.\right. \\
& +\left(Z^{(1)}+Z^{(2)} \int_{r} e^{i r x} \eta_{r}\right) \int_{s} e^{i s x} \eta_{s}\left(q \cdot s\left(K_{s}^{-1}+K_{q}^{-1}\right)+p \cdot s\left(K_{s}^{-1}+K_{p}^{-1}\right)\right) \\
& \left.+p \cdot q\left(Z+Z^{(1)} \int_{r} e^{i r x} \eta_{r}+\frac{1}{2} Z^{(2)} \int_{r, s} e^{i(r+s) x} \eta_{r} \eta_{s}\right)\left(K_{p}^{-1}+K_{q}^{-1}\right)\right] \\
& \left.+\left(U^{(2)}+U^{(3)} \int_{r} e^{i r x} \eta_{r}+\frac{1}{2} U^{(4)} \int_{r, s} e^{i(r+s) x} \eta_{r} \eta_{s}\right)\right\} \\
= & \delta_{p+q, 0}\left[U^{(2)}-\frac{1}{2} Z p \cdot q\left(K_{p}^{-1}+K_{q}^{-1}\right)\right] \\
& +\int_{r} \delta_{p+q+r, 0} \eta_{r}\left[U^{(3)}-\frac{1}{2} Z^{(1)}\left(p \cdot q\left(K_{p}^{-1}+K_{q}^{-1}\right)\right.\right. \\
& \left.\left.+q \cdot r\left(K_{r}^{-1}+K_{q}^{-1}\right)+p \cdot r\left(K_{r}^{-1}+K_{p}^{-1}\right)\right)\right] \\
& +\frac{1}{2} \int_{r, s} \delta_{p+q+r+s, 0} \eta_{r} \eta_{s}\left[U^{(4)}-Z^{(2)}\left(\frac{1}{2} p \cdot q\left(K_{p}^{-1}+K_{q}^{-1}\right)+r \cdot s K_{s}^{-1}\right.\right. \\
& \left.\left.+q \cdot s\left(K_{s}^{-1}+K_{q}^{-1}\right)+p \cdot r\left(K_{p}^{-1}+K_{r}^{-1}\right)\right)\right]
\end{aligned}
$$

where $Z^{(n)}=Z^{(n)}(\Phi), U^{(n)}=U^{(n)}(\Phi)$ and $K_{s}=K\left(s^{2}\right)$.

WH equation in $\mathcal{O}\left(\eta^{2}\right)$ : We set $K=1$ in (26), use sharp cut-off, i.e. all momentum integral is for $k-\Delta k<|p|<k$,

$$
\begin{aligned}
V^{2} \frac{\delta^{2} S}{\delta \phi_{p} \delta \phi_{q}}= & \delta_{p+q, 0}\left(U^{(2)}-Z p \cdot q\right)+\int_{r} \delta_{p+q+r, 0} \eta_{r}\left[U^{(3)}-Z^{(1)}(p \cdot q+q \cdot r+p \cdot r)\right] \\
& +\frac{1}{2} \int_{r, s} \delta_{p+q+r+s, 0} \eta_{r} \eta_{s}\left[U^{(4)}-Z^{(2)}(p \cdot q+r \cdot s+q \cdot s+p \cdot s+q \cdot r+p \cdot r)\right] \\
= & G_{p, q}^{-1}+A_{p, q}+B_{p, q},
\end{aligned}
$$

with

$$
\begin{aligned}
G_{p, q}^{-1} & =\delta_{p+q, 0}\left[U^{(2)}+\frac{1}{2} Z\left(p^{2}+q^{2}\right)\right], \\
A_{p, q} & =\int_{r} \delta_{p+q+r, 0} \eta_{r}\left[U^{(3)}+\frac{1}{2} Z^{(1)}\left(p^{2}+q^{2}+r^{2}\right)\right], \\
B_{p, q} & =\frac{1}{2} \int_{r, s} \delta_{p+q+r+s, 0} \eta_{r} \eta_{s}\left[U^{(4)}+\frac{1}{2} Z^{(2)}\left(p^{2}+q^{2}+r^{2}+s^{2}\right)\right] .
\end{aligned}
$$

As emphasised above, the logarithmic function on the right hand side of the WH equation is computed within the space of field configurations whose Fourier transform is non-vanishing for $k-\Delta k<|p|<k$ only. In order to keep this constrain in an explicit manner we introduce the projection operator $\mathcal{P}$ corresponding to this space and write the rest of the $\mathcal{O}\left(\eta^{2}\right)$ evolution equation as

$$
\partial_{k} S[\phi]=-\frac{\hbar}{2 \Delta k} \operatorname{tr} \log \left[G^{-1}+A+B\right]
$$




$$
=-\frac{\hbar}{2 \Delta k} \operatorname{tr} \mathcal{P} \log G^{-1}-\frac{\hbar}{2 \Delta k} \operatorname{tr}[\mathcal{P} G(A+B)]+\frac{\hbar}{4 \Delta k} \operatorname{tr}[\mathcal{P} G A \mathcal{P} G A]
$$

Since the propagator does not lead out from the function space, $[\mathcal{P}, G]=0$, it is sufficient to make the replacement $G \rightarrow \mathcal{P} G$ on the right hand side of the evolution equation. The term $\mathcal{O}(\eta)$ drops owing to the vanishing of the homogeneous component of the fluctuation, $\eta_{p=0}=0$ and we find

$$
\begin{aligned}
\frac{1}{2} \int_{r} \eta_{-r} \eta_{r}\left[\dot{Z} r^{2}+\dot{U}^{(2)}\right]+V \dot{U}= & -\frac{\hbar k^{d}}{2(2 \pi)^{d}} \int d e\left\{V \log \left[Z k^{2}+U^{(2)}\right]\right. \\
& -\int_{r} \eta_{-r} \eta_{r} \mathcal{P}_{r-e k, e k-r} \frac{\left[U^{(3)}+\frac{1}{2} Z^{(1)}\left(k^{2}+(e k-r)^{2}+r^{2}\right)\right]^{2}}{2\left(Z k^{2}+U^{(2)}\right)\left(Z(e k-r)^{2}+U^{(2)}\right)} \\
& \left.+\int_{r} \eta_{-r} \eta_{r} \frac{U^{(4)}+Z^{(2)}\left(k^{2}+r^{2}\right)}{2\left(Z k^{2}+U^{(2)}\right)}\right\}
\end{aligned}
$$

including an integration over the direction of $k$, the unit vector $e$.

The identification of the terms $\mathcal{O}\left(\eta^{0} r^{0}\right)$ results the equations

$$
\dot{U}=-\frac{\hbar k^{d} \Omega_{d}}{2(2 \pi)^{d}} \log \left[Z k^{2}+U^{(2)}\right]
$$

c.f. Eq. (14). The terms $\mathcal{O}\left(\eta^{2} r^{2}\right)$ give

$$
\begin{aligned}
\dot{Z} \int_{r} \eta_{-r} \eta_{r} r^{2}= & -\frac{\hbar k^{d}}{2(2 \pi)^{d}} \int_{r} \frac{\eta_{-r} \eta_{r}}{Z k^{2}+U^{(2)}} \int d e\left\{Z^{(2)} r^{2}\right. \\
& -\mathcal{P}_{r-e k, e k-r}\left[2 r^{2} Z^{(1)} \frac{U^{(3)}+Z^{(1)} k^{2}}{Z k^{2}+U^{(2)}}-Z r^{2} \frac{\left(U^{(3)}+Z^{(1)} k^{2}\right)^{2}}{\left(Z k^{2}+U^{(2)}\right)^{2}}\right. \\
& \left.\left.+k^{2}(e r)^{2}\left(\frac{Z^{(1) 2}}{Z k^{2}+U^{(2)}}-Z Z^{(1)} \frac{U^{(3)}+Z^{(1)} k^{2}}{\left(Z k^{2}+U^{(2)}\right)^{2}}\right)\right]\right\} .
\end{aligned}
$$

Finally, in $\mathcal{O}\left(\eta^{2} r^{0}\right)$ we find

$$
\begin{aligned}
\dot{U}^{(2)} \int_{r} \eta_{-r} \eta_{r}= & -\frac{\hbar k^{d}}{2(2 \pi)^{d}} \int_{r} \frac{\eta_{-r} \eta_{r}}{Z k^{2}+U^{(2)}} \int d e\left[Z^{(2)} k^{2}\right. \\
& \left.+U^{(4)}-\mathcal{P}_{r-e k, e k-r} \frac{\left(Z^{(1)} k^{2}+U^{(3)}\right)^{2}}{Z k^{2}+U^{(2)}}\right] .
\end{aligned}
$$

Let us make the replacement $\mathcal{P}_{r-k, k-r} \rightarrow 1+\left[\mathcal{P}_{r-k, k-r}-1\right]$ in Eqs. (35)-(37) and call the contributions corresponding to 1 and $\mathcal{P}_{k-r, k-r}-1$ regular and irregular, respectively. The regular contribution can be obtained from the one-loop graphs. This has already been seen for Eq. (35) and the regular part of Eq. (37) is just the second derivative of Eq. (35).

But there are problems with the irregular contributions which represent the cut-off in the multi-loop integrals. One obvious problem is the inconsistency between Eqs. (35) and (37). Another problem is that the irregular contributions include a truncated $r$ integration and as a result the left and right hand sides of the equations have different 
$\eta$-dependence. This could be avoided by imposing $r<<\Delta k$ for the momentum of the fluctuations. But the price is unacceptable high since it would indicate that the radius of convergence for the gradient expansion is smaller then the infinitesimal $\Delta k$.

Another problem the projection operator leads to is non-locality. It is known that the bare action is non-local at the scale of the U.V. cut-off, i.e. it contains higher order derivatives up to a finite order. In fact, the gradient $\partial^{n} / \partial x^{\mu n}$ represents couplings up to $n$ lattice spacing distances when lattice regularization is used. But terms in the action which are non-polynomial in the gradient induce correlations at finite, i.e. cut-off independent distances and are not acceptable. Returning to the gradient expansion we note that the operator $\mathcal{P}_{r-k, k-r}$ restricts the integration domain for $r$ in such a non-isotropical manner that not only $r^{2}$ but $|r|$ appears, too. This latter corresponds to the non-local operator $\sqrt{\square}$ in the gradient expansion.

This problem, inherent in the sharp cut-off procedure in the momentum space is rather general. The one-loop correction for the two-particle scattering amplitude in the $\phi^{4}$ model, represented by the fourth graph in Fig. 3c has a non-local contribution when the momenta in both internal lines are properly restricted by the U.V. cut-off. Such nonlocal effects, disregarded in the usual textbooks, might well be negligible in a renormalized theory because they are represented by non-renormalizable, irrelevant operators such as $\phi^{3} \sqrt{\square} \phi$. Therefore one hopes that their effects become weak when the cut-off is removed. The more careful analysis is rather involved since the details of the cut-off procedure within the momentum regime $(1-\epsilon) k<p<(1+\epsilon) k$ influences the dynamics on the distance scale $\hbar /(2 \epsilon k)$ which is a quantity of the form $0 \cdot \infty$.

But it is not so easy to dispel the doubts. First, the irrelevance and unimportance of a coupling constant are quite different concepts. An irrelevant coupling constant approaches its I.R. fixed point value as we move in the I.R. direction. But its value at the fixed point may be strong, indicating that the coupling constant in question is important. It is the scale dependence only what becomes unimportant for an irrelevant coupling constant and not its presence. The higher order vertices induced at low energies in a strongly coupled self interacting scalar field theory in $d<4$ may serve as an example of this difference. Second, the global view of the renormalization group, outlined in Section 3.2 suggest that a coupling constant which is irrelevant at the U.V. fixed point does not necessarily remain so at around the I.R. fixed point. Finally, this problem is obviously present in effective theories where the cut-off reaches physical scales.

\subsection{Resumming the perturbation expansion}

The basic idea leading to the WH equation is the successive elimination of the degrees of freedom. This procedure produces the blocked action, the integrand of the functional integral of the blocked theory. This integrand must be well defined, ie each mode must either be left intact or be completely eliminated during the blocking. This is the positive side of the sharp cut-off in momentum space. The negative side is that it generates nonlocal interactions and spoils the gradient expansion. The simplest cure, to smear out the 
regulator and to use smooth cut-off procedure, is not a valid alternative. This is because the blocking based on smooth cut-off suppresses the modes partially and the integrand for the functional integral of the blocked theory is ill defined. Despite this general sounding argument one can proceed and generalize the successive elimination process for smooth cut-off in a rather surprising manner.

\subsubsection{Polchinski equation}

Let us start with the partition function

$$
Z_{k}=\int D[\Phi] e^{-\frac{1}{2 \hbar} \Phi G_{k}^{-1} \Phi-\frac{1}{\hbar} S_{k}^{I}[\Phi]},
$$

where the interaction functional $S_{k}^{I}[\Phi]$ corresponds to the U.V. cut-off $k$. We split the propagator and the field variable into IR and U.V. components,

$$
G_{k}=G_{k-\Delta k}+\tilde{G}_{k}, \Phi=\phi+\tilde{\phi},
$$

with the intention that the fields $\phi$ and $\tilde{\phi}$ should propagate with $G_{k-\Delta k}$ and $\tilde{G}_{k}$, respectively. In other words, the kinetic energy contribution to the action is supposed to be of the form $\left(\phi G_{k-\Delta k}^{-1} \phi+\frac{1}{2} \tilde{\phi} \tilde{G}_{k}^{-1} \tilde{\phi}\right) / 2$ when written in terms of $\phi$ and $\tilde{\phi}$.

The introduction of the fields $\phi$ and $\tilde{\phi}$ separated by a smooth cut-off makes both $\phi_{p}$ and $\tilde{\phi}_{p}$ non-vanishing and appears as a double counting of the degrees of freedom. In order to ensure the proper integration measure we introduce a dummy field, $\tilde{\Phi}$, in such a manner that a suitable, momentum dependent linear combination of $\phi_{p}$ and $\tilde{\phi}_{p}$ reproduces $\Phi_{p}$ and $\tilde{\Phi}_{p}$

$$
\left(\begin{array}{c}
\Phi \\
\tilde{\Phi}
\end{array}\right)=\left(\begin{array}{cc}
A_{1,1}(\square) & A_{1,2}(\square) \\
A_{2,1}(\square) & A_{2,2}(\square)
\end{array}\right)\left(\begin{array}{c}
\phi \\
\tilde{\phi}
\end{array}\right)
$$

and decouple $\tilde{\Phi}$

$$
\frac{1}{2} \phi G_{k-\Delta k}^{-1} \phi+\frac{1}{2} \tilde{\phi} \tilde{G}_{k}^{-1} \tilde{\phi}=\frac{1}{2} \Phi G_{k}^{-1} \Phi+\frac{1}{2} \tilde{\Phi} \tilde{G}_{D}^{-1} \tilde{\Phi}
$$

with a freely chosen dummy propagator $\tilde{G}_{D}$. Owing to the second equation in (39) $A_{1,1}=A_{1,2}=1$. The condition (41) gives

$$
\left(\begin{array}{cc}
G_{k-\Delta k}^{-1} & 0 \\
0 & \tilde{G}_{k}^{-1}
\end{array}\right)=\left(\begin{array}{cc}
1 & A_{2,1}(\square) \\
1 & A_{2,2}(\square)
\end{array}\right)\left(\begin{array}{cc}
G_{k}^{-1} & 0 \\
0 & \tilde{G}_{D}^{-1}
\end{array}\right)\left(\begin{array}{cc}
1 & 1 \\
A_{2,1}(\square) & A_{2,2}(\square)
\end{array}\right)
$$

whose solution is $A_{2,1}(\square)=\sqrt{\tilde{G}_{D} \tilde{G}_{k} / G_{k-\Delta k} G_{k}}, A_{2,2}(\square)=\sqrt{\tilde{G}_{D} G_{k-\Delta k} / \tilde{G}_{k} G_{k}}$. The transformation (40) is non-singular so long as $\tilde{G}_{k} \neq G_{k-\Delta k}$, the propagations of $\phi$ and $\tilde{\phi}$ are distinguishable.

We are finally in the position to describe the blocking procedure which consists of the following steps:

(1) Redouble the number of degrees of freedom, $\Phi \rightarrow(\Phi, \tilde{\Phi})$.

(2) Rotate the new, dummy degrees of freedom into the dynamics in such manner that the rotated fields split the original field variable and follow the prescribed propagation. 
(3) Integrate out $\tilde{\phi}$.

In order to complete step 3. we insert a field independent constant into the partition function,

$$
Z_{k}=\int D[\Phi] D[\tilde{\Phi}] e^{-\frac{1}{2 \hbar} \tilde{\Phi} \tilde{G}_{D}^{-1} \tilde{\Phi}-\frac{1}{2 \hbar} \Phi G_{k}^{-1} \Phi-\frac{1}{\hbar} S_{k}^{I}[\Phi]} .
$$

The redistribution of the degrees of freedom by the inverse of the transformation (40) yields

$$
Z_{k}=\int D[\phi] D[\tilde{\phi}] e^{-\frac{1}{2 \hbar} \phi G_{k-\Delta k}^{-1} \phi-\frac{1}{2 \hbar} \tilde{\phi} \tilde{G}_{k}^{-1} \tilde{\phi}-\frac{1}{\hbar} S_{k}^{I}[\phi+\tilde{\phi}]},
$$

up to a constant. This motivates the introduction of the blocked action $S_{k-\Delta k}[\phi]$ defined as

$$
e^{-S_{k-\Delta k}[\phi]}=\int D[\tilde{\phi}] e^{-\frac{1}{2 \hbar} \tilde{\phi} \tilde{G}_{k}^{-1} \tilde{\phi}-\frac{1}{\hbar} S_{k}^{I}[\phi+\tilde{\phi}]} .
$$

The higher loop corrections were suppressed in the WH equation by restricting the functional space of the blocking, the volume of the loop integration in momentum space. But there is another way to suppress the radiative corrections, to decrease the propagator. Such a suppression method is better suited for smooth cut-off where the U.V. field can not be constrained into a restricted, 'small' functional space. This strategy is implemented by requiring that in the so far arbitrary split $(39) \tilde{G}_{k}(p)=\mathcal{O}(\Delta k)$. The obvious choice is

$$
\tilde{G}_{k}(p)=\Delta k \partial_{k} G_{k}(p) .
$$

By assuming the convergence of the perturbation expansion we expand the action in $\tilde{\phi}$ and perform the integration over $\tilde{\phi}$. Since the new small parameter is the propagator we retain graphs where the propagator appears in a minimal number. By assuming that the saddle point is at most $\mathcal{O}(\Delta k)$ we can expand the action in $\tilde{\phi}$,

$$
\begin{aligned}
e^{-\frac{1}{\hbar} S_{k-\Delta k}^{I}[\phi]} & =\int D[\tilde{\phi}] e^{-\frac{1}{2 \hbar} \tilde{\phi} \tilde{G}_{k}^{-1} \tilde{\phi}-\frac{1}{\hbar} S_{k}^{I}[\phi]-\frac{1}{\hbar} \tilde{\phi} \cdot \frac{\delta S_{k}^{I}[\phi]}{\delta \phi}-\frac{1}{2 \hbar} \tilde{\phi} \cdot \frac{\delta^{2} S_{k}^{I}[\phi]}{\delta \phi \delta \phi} \cdot \tilde{\phi}+\mathcal{O}\left(\tilde{\phi}^{3}\right)} \\
& =e^{-\frac{1}{\hbar} S_{k}^{I}[\phi]+\frac{1}{2 \hbar} \frac{\delta S_{k}^{I}[\phi]}{\delta \phi} \cdot\left(\tilde{G}_{k}^{-1}+\frac{\delta^{2} S_{k}^{I}[\phi]}{\delta \phi \delta \phi}\right)^{-1} \cdot \frac{\delta S_{k}^{I}[\phi]}{\delta \phi}-\frac{1}{2} \operatorname{tr} \log \left(\tilde{G}_{k}^{-1}+\frac{\delta^{2} S_{k}^{I}[\phi]}{\delta \phi \delta \phi}\right)+\mathcal{O}\left(\Delta k^{2}\right)} \\
& =e^{-\frac{1}{\hbar} S_{k}^{I}[\phi]+\frac{\Delta k}{\hbar}\left[\frac{1}{2} \frac{\delta S_{k}^{I}[\phi]}{\delta \phi} \cdot \partial_{k} G_{k} \cdot \frac{\delta S_{k}^{I}[\phi]}{\delta \phi}-\frac{\hbar}{2} \operatorname{tr}\left(\partial_{k} G_{k} \frac{\delta^{2} S_{k}^{I}[\phi]}{\delta \phi \delta \phi}\right)\right]+\mathcal{O}\left(\Delta k^{2}\right)} \\
& =\left[1-\frac{\hbar \Delta k}{2} \frac{\delta}{\delta \phi} \cdot \partial_{k} G_{k} \cdot \frac{\delta}{\delta \phi}\right] e^{-\frac{1}{\hbar} S_{k}^{I}[\phi]}+\mathcal{O}\left(\Delta k^{2}\right)
\end{aligned}
$$

The third equation was obtained by expanding the logarithmic function and gives the differential equation [9], [10]

$$
\partial_{k} S_{k}^{I}[\phi]=\frac{1}{2} \frac{\delta S_{k}^{I}[\phi]}{\delta \phi} \cdot \partial_{k} G_{k} \cdot \frac{\delta S_{k}^{I}[\phi]}{\delta \phi}-\frac{\hbar}{2} \operatorname{tr}\left[\partial_{k} G_{k} \frac{\delta^{2} S_{k}^{I}[\phi]}{\delta \phi \delta \phi}\right] .
$$

The fourth line leads to a linear equation,

$$
k \partial_{k} e^{-\frac{1}{\hbar} S_{k}^{I}[\phi]}=\hbar \mathcal{B} e^{-\frac{1}{\hbar} S_{k}^{I}[\phi]}, \quad \mathcal{B}=\frac{1}{2} \frac{\delta}{\delta \phi} \cdot k \partial_{k} G_{k} \cdot \frac{\delta}{\delta \phi} .
$$

It is instructive to notice the similarities and differences with the WH equation. Eq. (45) looks as the starting point for the derivation of the WH equation and could have 
been obtained in a trivial manner for sharp cut-off. The crux of the argument leading to this relation is that this simple-looking equation is actually valid for smooth cut-off. But in a surprising manner it is just the sharp cut-off limit when the rest of the derivation of Polchinksi equation is invalid. This is because $\tilde{G}_{k}$ as given in (46) is $\Delta k \mathcal{O}\left(\Delta k^{-1}\right)=$ $\mathcal{O}\left(\Delta k^{0}\right)$.

Eq. (48) is a leading order equation in the perturbation rather than the loop expansion. It is formally similar to the WH equation, (10) when only the leading order is retained in the expansion (11) which corresponds to keeping graphs with a single vertex only in Fig. 1(b) and using the dashed lines to denote the propagation of $\tilde{\phi}$. Notice that even if the higher order contributions of the perturbation expansion are suppressed in the limit $\Delta k \rightarrow 0$, the convergence of the perturbation expansion was assumed.

The difference between WH equation and (48) is that the tree-level first term on the right hand side is always non-vanishing in Eq. (48), contrary to the case of the WH equation. It is easier to understand the reason of the non-trivial saddle point when the blocking (6) is considered in the presence of the source term. The source can be omitted and the cut-off independence of a single quantity, the partition function can be considered as the basic equation when the source has vanishing component in the functional subspace to integrate over in the blocking. In case of a smooth cut-off the background field has arbitrary momentum component and we can not really omit the source. One ought to follow the more cumbersome blocking which keeps the generator functional RG invariant. When classical physics (action) is modified by changing the cut-off, the field induced by the source changes, as well. This is the origin of the tree-level renormalization. But we made a short-cut and omitted the source. The price is that the tree-level renormalization can only be understood by letting the background field, $\phi$ vary freely.

The understanding how the $\phi$-dependence may give rise the tree-level renormalization in (47) is an interesting demonstration of the well know theorem stating that the expansion in loops and in $\hbar$ are equivalent. In fact, the first equation in (47) introduces an effective theory for $\tilde{\phi}$ and the first two terms in the exponent of the second line give the effective action corresponding to a given background field $\phi$ in the classical approximation. In other words, the action with lowered cut-off which is supposed to reproduce the action with the original cut-off for arbitrary configuration $\phi$ requires tree-level adjustment. The second term in the exponent has two factors of $\hbar^{-1}$ coming from the 'source' $\hbar^{-1} \delta S_{k}^{I}[\phi] / \delta \phi$ of the field $\tilde{\phi}$ and a factor of $\hbar$ from the $\tilde{\phi}$ propagator. The overall $1 / \hbar$ disappears in the differential equation (48) which displays $\hbar$ times the exponent. This is the way the graphs of Fig. 1(b) appear on the $\mathcal{O}\left(\hbar^{0}\right)$ tree-level in Eq. (48). Another reason is that they are graphs with no freely changing loop-momentum variable. One can understand in a similar manner that both the tree- and the loop-level graphs appear with a common factor of $\hbar$ in Eq. (49). 


\subsubsection{Gradient expansion}

We shall use here the results obtained in section 2.1.3 with the ansatz

$$
S^{I}[\phi]=\int_{x}\left[\frac{1}{2}\left(Z\left(\phi_{x}\right)-1\right) \partial_{\mu} \phi_{x} K^{-1}\left(-\square / k^{2}\right) \partial_{\mu} \phi_{x}+U\left(\phi_{x}\right)\right]
$$

to derive the gradient expansion for Eq. (48). For the left hand side we find in the given order of the gradient expansion

$$
\begin{aligned}
& \frac{\dot{Z}}{2} \int_{p} \eta_{-p} \eta_{p} \frac{p^{2}}{K_{p}}+\frac{Z-1}{2} \int_{p} \eta_{-p} \eta_{p} p^{2} \dot{K}_{p}^{-1}+V \dot{U}+\frac{\dot{U}^{(2)}}{2} \int_{p} \eta_{-p} \eta_{p} \\
& \approx \frac{\dot{Z}}{2} \int_{p} \eta_{-p} \eta_{p} \frac{p^{2}}{K_{p}}+V \dot{U}+\frac{\dot{U}^{(2)}}{2} \int_{p} \eta_{-p} \eta_{p} .
\end{aligned}
$$

By means of the relation $k \partial_{k} G=-2 K_{p}^{\prime} / k^{2}$ where $K_{p}^{\prime}=d K(z) / d z, z=p^{2} / k^{2}$ the right hand side gives

$$
\begin{aligned}
& -\int_{p}\left\{\delta_{p, 0} U^{(1)}+\eta_{p}\left(U^{(2)}+(Z-1) \frac{p^{2}}{K_{p}}\right)+\frac{1}{2} \int_{r} \eta_{r} \eta_{p-r}\left[U^{(3)}-Z^{(1)}\left(\frac{r \cdot(p-r)}{K_{p-r}}-\frac{p \cdot r}{K_{r}}-\frac{p \cdot(p-r)}{K_{p}}\right)\right]\right\} \\
& \times \frac{K_{p}^{\prime}}{k^{2}}\left\{\delta_{p, 0} U^{(1)}+\eta_{-p}\left(U^{(2)}+(Z-1) \frac{p^{2}}{K_{p}}\right)+\frac{1}{2} \int_{s} \eta_{-p-s} \eta_{s}\left[U^{(3)}-Z^{(1)}\left(\frac{p \cdot s}{K_{p}}-\frac{(p+s) \cdot s}{K_{s}}-\frac{p \cdot(p+s)}{K_{-p-s}}\right)\right]\right\} \\
& +\hbar \int_{p} \frac{K_{p}^{\prime}}{k^{2}}\left\{V\left(U^{(2)}+(Z-1) \frac{p^{2}}{K_{p}}\right)+\frac{1}{2} \int_{r} \eta_{-r} \eta_{r}\left[U^{(4)}+Z^{(2)}\left(\frac{p^{2}}{K_{p}}+\frac{r^{2}}{K_{r}}\right)\right]\right\} \\
& \approx-V U^{(1) 2} \frac{K_{0}^{\prime}}{k^{2}}-\int_{r} \eta_{-r} \eta_{r}\left[\frac{K_{0}^{\prime}+K_{0}^{\prime \prime} K_{0} r^{2} /\left(k^{2} K_{r}\right)}{k^{2}}\left(U^{(2)}+(Z-1) \frac{r^{2}}{K_{r}}\right)^{2}+\frac{K_{0}^{\prime}}{k^{2}} U^{(1)}\left(U^{(3)}+Z^{(1)} \frac{r^{2}}{K_{r}}\right)\right] \\
& +\hbar \int_{p} \frac{K_{p}^{\prime}}{k^{2}}\left\{V\left(U^{(2)}+(Z-1) \frac{p^{2}}{K_{p}}\right)+\frac{1}{2} \int_{r} \eta_{-r} \eta_{r}\left[U^{(4)}+Z^{(2)}\left(\frac{p^{2}}{K_{p}}+\frac{r^{2}}{K_{r}}\right)\right]\right\}
\end{aligned}
$$

This equation is consistent since the terms $\mathcal{O}\left(\eta^{2}\right)$ are comparable thanks to the smoothness of the cut-off. We compare the coefficients of the same $\eta$-dependent expressions and find

$$
\begin{aligned}
& k^{2} \dot{U}=-U^{(1) 2} K_{0}^{\prime}+\hbar \int_{p} K_{p}^{\prime}\left(U^{(2)}+(Z-1) \frac{p^{2}}{K_{p}}\right) \\
& k^{2} \dot{Z}=-4 K_{0}^{\prime} U^{(2)}(Z-1)-2 K_{0}^{\prime} U^{(1)} Z^{(1)}-2 \frac{K_{0}^{\prime \prime} K_{0}}{k^{2}} U^{(2) 2}+\hbar Z^{(2)} \int_{p} K_{p}^{\prime},
\end{aligned}
$$

and

$$
k^{2} \dot{U}^{(2)}=-2 K_{0}^{\prime} U^{(2) 2}-2 K_{0}^{\prime} U^{(1)} U^{(3)}+\hbar \int_{p} K_{p}^{\prime}\left(U^{(4)}+Z^{(2)} \frac{p^{2}}{K_{p}}\right)
$$

We have two independent equations for two functions because the Eq. (54) follows from the first equation of (53).

It is instructive to check the first equation in the local potential approximation $Z=1$,

$$
\dot{U}=\frac{U^{(1) 2}}{2} \frac{k \partial_{k} K_{p}}{p^{2}} \underset{\mid p=0}{2}-\frac{\hbar U^{(2)}}{2} \frac{k \partial_{k} K_{p}}{p^{2}} .
$$


The first term describes the tree-level adjustment of the bare action in keeping the physics cut-off independent and some of the corresponding graphs are depicted in Fig. 1(a). The present form, characteristic of the gradient expansion, is non-vanishing when the change of the cut-off at $p=k$ is felt at the base point of the gradient expansion, $p=0$. The second term is the leading order perturbative contribution to the WH equation (14) when the mass term is treated perturbatively, as expected and the beta functions,

$$
\beta_{n}(\phi)=-\frac{\hbar U^{(n+2)}}{2} \int_{p} \frac{k \partial_{k} K_{p}}{p^{2}}
$$

for $n>2$ correspond to the leading order first column in Eqs. (23) with $g_{2}=0$. The independent mode approximation reproduces the leading order renormalization of the coupling constants and the numerical integration resums the perturbation expansion in the approximation where the effective vertices are replaced by their values at $p=0$. The leading order perturbative contributions, retained in the RG equation are linear in the coupling constants and the classical, non-linear terms drop out in the linearized evolution around the Gaussian fixed point therefore the usual critical exponents are reproduced.

\subsection{Composite operator renormalization}

The renormalization of composite operators [11] seems to be a highly technical and subtle issue, dealing with the removal of the U.V. divergences from Green functions where composite operators are inserted. But it becomes more elementary and general [12] as soon as we are ready to give up the perturbative approach and can reformulate the procedure in a general, non-perturbative manner. In order to see this let us go back to the remark made at Eq. (6). It is not enough to impose the RG invariance of the partition function only since it yields a single equation for infinitely many coupling constants. Instead one should require the RG invariance of the generator functional for Green functions because any observable can be reconstructed from this functional. This turns out to be rather cumbersome due to the source dependence generated for the blocked action [13]. We took another direction by imposing the RG invariance of another functional, the blocked action, cf Eqs. (7) and (45). Instead of this strategy we shall now use the matching of observables computed at different values of the cut-off to rederive the RG equation. This is a non-perturbative, blocking inspired generalization of the multiplicative RG schemes mentioned in the Introduction in what the scale dependence of the observables is followed instead of those of non-physical bare coupling constants.

We start with a toy model to demonstrate the natural relation between blocking and composite operator renormalization and the field theoretical application follows next by generalizing the source term in the generator functional. This term which usually involves the elementary field only is extended here for any local operator with inhomogeneous source and we consider it as part of the action which now contains inhomogeneous coupling constants. Our main point is that the beta functions corresponding to this extended action provide a bridge between the blocking and the composite operator renormalization. 


\subsubsection{Toy model}

Consider the two dimensional integral

$$
Z=\int d x d y e^{-S(x, y)}
$$

over $x$ and $y$ which play the role of low- and high-frequency variables, respectively, where the bare action is given by the expression

$$
S(x, y)=\frac{1}{2} s_{x} x^{2}+\frac{1}{2} s_{y} y^{2}+\sum_{n=0}^{\infty} g_{n}(x+y)^{n}
$$

in terms of bare coupling constants $g_{n}$. We use $\hbar=1$ in this section. The blocked action is defined as

$$
e^{-S(x)}=\int d y e^{-S(x, y)}
$$

The elementary, bare operators are $(x+y)^{n}$ with $n=0,1,2, \ldots$ and their expectation values are

$$
\frac{\int d x d y(x+y)^{n} e^{-S(x, y)}}{\int d x d y e^{-S(x, y)}}=\frac{\int d x \frac{\partial S(x)}{\partial g_{n}} e^{-S(x)}}{\int d x e^{-S(x)}} .
$$

The right hand side of this equation expresses the expectation value of the bare operator in terms of an operator of the blocked, 'thinner' system. The action $S(x)$ can be expanded in the terms of the operators $x^{n}$,

$$
S(x)=\frac{1}{2} s_{x} x^{2}+\sum_{m=0}^{\infty} g_{m}^{\prime} x^{m}
$$

giving rise the composite operators

$$
\left\{x^{n}\right\}=\frac{\partial S(x)}{\partial g_{n}}=\sum_{m=0}^{\infty} x^{m} S_{m, n}
$$

where

$$
S_{m, n}=\frac{\partial g_{m}^{\prime}}{\partial g_{n}}
$$

We have chosen a basis for operators in the bare theory and searched for operators in the blocked theory. The opposite question, the construction of the composite operators $\left[(x+y)^{n}\right]$ of the bare theory which reproduce the expectation values of the blocked operators gives

$$
\frac{\int d x d y\left[(x+y)^{n}\right] e^{-S(x, y)}}{\int d x d y e^{-S(x, y)}}=\frac{\int d x x^{n} e^{-S(x)}}{\int d x e^{-S(x)}},
$$

with

$$
\left[(x+y)^{n}\right]=\sum_{m=0}^{\infty}(x+y)^{m}\left(S^{-1}\right)_{m, n} .
$$

It is instructive to generalize the toy model for three variables,

$$
S(x, y, z)=\frac{1}{2} s_{x} x^{2}+\frac{1}{2} s_{y} y^{2}+\frac{1}{2} s_{z} z^{2}+\sum_{n=0}^{\infty} g_{n}(x+y+z)^{n},
$$


with

$$
e^{-S(x, y)}=\int d z e^{-S(x, y, z)}, \quad e^{-S(x)}=\int d y e^{-S(x, y)},
$$

where the operator mixing looks like

$$
\begin{aligned}
& \left\{x^{n}\right\}_{z}=\frac{\partial S(x, y, z)}{\partial g_{n}}=(x+y+z)^{n}, \\
& \left\{x^{n}\right\}_{y}=\frac{\partial S(x, y)}{\partial g_{n}}=-\frac{\frac{\partial}{\partial g_{n}} \int d z e^{-S(x, y, z)}}{\int d z e^{-S(x, y, z)}} \\
& \left\{x^{n}\right\}_{x}=\frac{\partial S(x)}{\partial g_{n}}=-\frac{\frac{\partial}{\partial g_{n}} \int d y d z e^{-S(x, y, z)}}{\int d y d z e^{-S(x, y, z)}} .
\end{aligned}
$$

These relations yield

$$
\left\{x^{n}\right\}_{y}=\frac{\int d z\left\{x^{n}\right\}_{z} e^{-S(x, y, z)}}{\int d z e^{-S(x, y, z)}}, \quad\left\{x^{n}\right\}_{x}=\frac{\int d y\left\{x^{n}\right\}_{y} e^{-S(x, y)}}{\int d y e^{-S(x, y)}},
$$

indicating that the evolution of the operators comes from the elimination of the field variable in their definition. We can compute the expectation value of $\left\{x^{n}\right\}$ at any level,

$$
\frac{\int d x d y d z\left\{x^{n}\right\}_{z} e^{-S(x, y, z)}}{\int d x d y d z e^{-S(x, y, z)}}=\frac{\int d x d y\left\{x^{n}\right\}_{y} e^{-S(x, y)}}{\int d x d y e^{-S(x, y)}}=\frac{\int d x\left\{x^{n}\right\}_{x} e^{-S(x)}}{\int d x e^{-S(x)}} .
$$

The lesson of this toy model is twofold. First, one sees that the formal definition of the renormalized operator at two different value of the cut-off differs because these operators are supposed to reproduce the same averages by means of different number of degrees of freedom. Second, it shows that the cut-off dependence of the renormalized operators can be obtained in a natural and simple manner by considering the bare coupling constants at one value of the cut-off as the functions of the coupling constants given at another value of the cut-off.

\subsubsection{Quantum Field Theory}

We generalize the operator mixing of the toy model for the scalar field theory given by the bare action

$$
S_{\Lambda}[\phi]=\int_{x} \sum_{n} G_{n, x}(\Lambda) O_{n}\left(\phi_{x}\right)=\sum_{\tilde{n}} G_{\tilde{n}}(\Lambda) O_{\tilde{n}}\left(\phi_{x}\right)
$$

where $O_{n}\left(\phi_{x}\right)$ represents a complete set of local operators (functions of $\phi_{x}$ and its spacetime derivatives) and $G_{n, x}(\Lambda)$ denotes the coupling constant. We simplify the expressions by introducing a single index $\tilde{n}$ for the pair $(n, x)$ labeling the basis elements for the local operators and $\sum_{\tilde{n}}=\sum_{n} \int_{x}$. The decomposition $\phi_{x}=\phi_{k, x}+\tilde{\phi}_{k, x}$ of the scalar field into a low- and high-frequency parts is carried out as in Eqs. (7) and (39).

The Kadanoff-Wilson blocking

$$
e^{-S_{k}\left[\phi_{k}\right]}=\int D\left[\tilde{\phi}_{k}\right] e^{-S_{\Lambda}\left[\phi_{k}+\tilde{\phi}_{k}\right]}
$$


for the action

$$
S_{k}\left[\phi_{k}\right]=\sum_{\tilde{n}} G_{\tilde{n}}(k) O_{\tilde{n}}\left(\phi_{k}\right)
$$

generates the RG flow

$$
k \partial_{k} G_{\tilde{n}}(k)=\beta_{\tilde{n}}(k, G) .
$$

The blocked operators are defined by

$$
\left\{O_{\tilde{n}}\left(\phi_{k}\right)\right\}_{k}=\frac{\delta S_{k}\left[\phi_{k}\right]}{\delta G_{\tilde{n}}(\Lambda)}=\frac{\int D\left[\tilde{\phi}_{k}\right] O_{\tilde{n}}\left(\phi_{k}+\tilde{\phi}_{k}\right) e^{-S_{\Lambda}\left[\phi_{k}+\tilde{\phi}_{k}\right]}}{\int D\left[\tilde{\phi}_{k}\right] e^{-S_{\Lambda}\left[\phi_{k}+\tilde{\phi}_{k}\right]}}
$$

in agreement with Eq. (68). They satisfy the equation

$$
\frac{\int D\left[\phi_{k}\right] \frac{\delta S_{k}\left[\phi_{k}\right]}{\delta G_{\tilde{n}}(\Lambda)} e^{-S_{k}\left[\phi_{k}\right]}}{\int D\left[\phi_{k}\right] e^{-S_{k}\left[\phi_{k}\right]}}=\frac{\int D\left[\phi_{k}\right] D\left[\tilde{\phi}_{k}\right] O_{\tilde{n}}\left(\phi_{k}+\tilde{\phi}_{k}\right) e^{-S_{\Lambda}\left[\phi_{k}+\tilde{\phi}_{k}\right]}}{\int D\left[\phi_{k}\right] D\left[\tilde{\phi}_{k}\right] e^{-S_{\Lambda}\left[\phi_{k}+\tilde{\phi}_{k}\right]}}
$$

c.f. Eq. (70), showing that they are represented in the effective theory by the functional derivative of the blocked action with respect to the microscopical coupling constants. The form (73) of the action gives the operator mixing

$$
\left\{O_{\tilde{n}}\left(\phi_{k}\right)\right\}_{k}=\sum_{\tilde{m}} \frac{\delta G_{\tilde{m}}(k)}{\delta G_{\tilde{n}}(\Lambda)} \frac{\delta S_{k}\left[\phi_{k}\right]}{\delta G_{\tilde{m}}(k)}=\sum_{\tilde{m}} O_{\tilde{m}}\left(\phi_{k}\right) S_{\tilde{m} \tilde{n}}(k, \Lambda)
$$

in a manner similar to Eq. (69). We introduced here the sensitivity matrix

$$
S_{\tilde{m}, \tilde{n}}(k, \Lambda)=\frac{\delta G_{\tilde{m}}(k)}{\delta G_{\tilde{n}}(\Lambda)} .
$$

It is the measure of the sensitivity of an effective strength of interaction on the initial condition of the RG trajectory, imposed in the U.V. domain, c.f. Eq. (153). The composite operator $\left\{O_{\tilde{n}}\left(\phi_{k}\right)\right\}_{k}$ introduced in Eq. (77) replaces the bare operator $\left\{O_{\tilde{n}}(\phi)\right\}_{\Lambda}$ in the Green functions of the effective theory for the I.R. modes $\phi_{k}$.

The differential equation generating the operator mixing (77) is obtained in the following manner. The relation

$$
G_{\tilde{n}}(k-\Delta k)=G_{\tilde{n}}(k)-\frac{\Delta k}{k} \beta_{\tilde{n}}(k, G)
$$

is used to arrive at the sensitivity matrix,

$$
\begin{aligned}
S_{\tilde{m}, \tilde{n}}(k-\Delta k, \Lambda)=\frac{\delta G_{\tilde{m}}(k-\Delta k)}{\delta G_{\tilde{n}}(\Lambda)} & =\sum_{\tilde{\ell}} \frac{\delta G_{\tilde{m}}(k-\Delta k)}{\delta G_{\tilde{\ell}}(k)} \frac{\delta G_{\tilde{\ell}}(k)}{\delta G_{\tilde{n}}(\Lambda)} \\
& =\sum_{\tilde{\ell}}\left[\delta_{\tilde{m} \tilde{\ell}}-\frac{\Delta k}{k} \frac{\delta \beta_{\tilde{m}}(k, G)}{\delta G_{\tilde{\ell}}(k)}\right] S_{\tilde{\ell}, \tilde{n}}(k)
\end{aligned}
$$

and to find

$$
k \partial_{k} S_{\tilde{m}, \tilde{n}}(k, \Lambda)=\sum_{\tilde{\ell}} \frac{\delta \beta_{\tilde{m}}(k, G)}{\delta G_{\tilde{\ell}}(k)} S_{\tilde{\ell}, \tilde{n}}(k, \Lambda)
$$


The scale dependence of the operator mixing matrix is governed by

$$
\gamma_{\tilde{n} \tilde{m}}(k)=\frac{1}{k} \frac{\delta \beta_{\tilde{n}}(k, G)}{\delta G_{\tilde{m}}(k)} .
$$

Summary: Eq. (74) represents the link between Kadanoff-Wilson blocking and composite operator renormalization. On the one hand, $\beta_{\tilde{n}}$ describes the evolution of the action in the traditional blocking scheme, Eq. (72). On the other hand, interpreting the coupling constants in the action as sources coupled to composite operators $\beta_{\tilde{n}}$ determines the mixing of composite operators in Eq. (82). Therefore all conepts and results of the blocking, e.g. fixed point, universality, etc. has a counterpart in composite operator renormalization.

\subsubsection{Parallel transport}

The operator mixing discussed above has a nice geometrical interpretation, a parallel transport of operators along the RG trajectory [14] with the connection $\Gamma_{\tilde{n}, \tilde{m}}(k)$. The independence of the expectation value from the cut-off,

$$
\frac{\int D\left[\phi_{k^{\prime}}\right] D\left[\tilde{\phi}_{k^{\prime}}\right] O_{\tilde{n}}\left(\phi_{k^{\prime}}+\tilde{\phi}_{k^{\prime}}\right) e^{-S_{k^{\prime}}\left[\phi_{k^{\prime}}+\tilde{\phi}_{k^{\prime}}\right]}}{\int D\left[\phi_{k^{\prime}}\right] D\left[\tilde{\phi}_{k^{\prime}}\right] e^{-S_{k^{\prime}}\left[\phi_{k^{\prime}}+\tilde{\phi}_{k^{\prime}}\right]}}=\frac{\int D\left[\phi_{k}\right] D\left[\tilde{\phi}_{k}\right] O_{\tilde{n}}\left(\phi_{k}+\tilde{\phi}_{k}\right) e^{-S_{k}\left[\phi_{k}+\tilde{\phi}_{k}\right]}}{\int D\left[\phi_{k}\right] D\left[\tilde{\phi}_{k}\right] e^{-S_{k}\left[\phi_{k}+\tilde{\phi}_{k}\right]}}
$$

defines the parallel transport of composite operators. The linearity of the mixing (77) assures that this parallel transport is indeed linear and can therefore be characterized by a covariant derivative

$$
D_{k} O_{k}=\left(\partial_{k}-\Gamma\right) O_{k}
$$

where $\Gamma_{\tilde{m}, \tilde{n}}=\left(S^{-1} \cdot \gamma \cdot S\right)_{\tilde{n}, \tilde{m}}$ in such a manner that $D_{k} O_{k}=0$ along the RG flow.

The dynamical origin of the connection comes from the fact that there are two different sources the scale dependence of $\left\langle O_{k}\right\rangle$ comes from: from the explicit $k$-dependence of the operator and from the implicit $k$-dependence due to the cut-off in the path integration. The operator mixing is to balance them. In fact, the covariant derivative could have been introduced by the relation

$$
\partial_{k}\left\langle O_{k}\right\rangle=\left\langle D_{k} O_{k}\right\rangle
$$

requiring that the operator mixing generated by the connection is to make up the implicit $k$-dependence of the expectation value coming from the cut-off.

It is obvious that $\Gamma$ is vanishing in the basis $\left\{O_{\tilde{n}}\right\}_{k}$,

$$
\partial_{k}\left\langle\sum_{\tilde{n}} c_{\tilde{n}}(k)\left\{O_{\tilde{n}}\right\}_{k}\right\rangle=\sum_{\tilde{n}} \partial_{k} c_{\tilde{n}}(k)\left\langle\left\{O_{\tilde{n}}\right\}_{k}\right\rangle=\left\langle\partial_{k} \sum_{\tilde{n}} c_{\tilde{n}}(k)\left\{O_{\tilde{n}}\right\}_{k}\right\rangle .
$$

The connection can in principle be found in any other basis by simple computation.

\subsubsection{Asymptotical scaling}

We linearize the beta-functions around a fixed point $G_{\tilde{m}}^{*}$,

$$
\beta_{\tilde{n}} \approx \sum_{\tilde{m}} \Gamma_{\tilde{n} \tilde{m}}^{*}\left(G_{\tilde{m}}-G_{\tilde{m}}^{*}\right)
$$


and write the scaling coupling constant, the left eigenvectors of $\Gamma$

$$
\sum_{\tilde{m}} c_{\tilde{n}, \tilde{m}}^{\text {left }} \Gamma_{\tilde{m}, \tilde{r}}^{*}=\alpha_{\tilde{n}} c_{\tilde{n}, \tilde{r}}^{\text {left }}
$$

as

$$
G_{\tilde{n}}^{s c}=\sum_{\tilde{m}} c_{\tilde{n}, \tilde{m}}^{\text {left }}\left(G_{\tilde{m}}-G_{\tilde{m}}^{*}\right) .
$$

They display the scale dependence

$$
G_{\tilde{n}}^{s c} \sim k^{\alpha_{\tilde{n}}}
$$

Furthermore let us fix the overall scale of the local operators. This can be done by using the decomposition

$$
O\left(\phi_{x}\right)=\sum_{n} b_{n} O_{n}\left(\phi_{x}\right)
$$

where $O_{n}(\phi(x))$ is the product of the terms $\partial_{\mu_{1}} \cdots \partial_{\mu_{\ell}} \phi^{m}(x)$ with coefficient 1 . The norm $\|O\|=\sqrt{\sum_{n} b_{n}^{2}}$ is introduced with the notation

$$
\bar{O}=\frac{O}{\|O\|}
$$

for the operators of unit norm. We shall use the convention that the coupling constants $G_{\tilde{n}}(\Lambda)$ always multiply operators of unit norm in the action.

The scaling operators

$$
O_{\tilde{n}}^{s c}=\sum_{\tilde{m}} c_{\tilde{n} \tilde{m}}^{\text {right }} O_{\tilde{m}}
$$

are obtained by means of the right eigenvectors of $\Gamma^{*}$,

$$
\sum_{\tilde{m}} \Gamma_{\tilde{r} \tilde{m}}^{*} c_{\tilde{m} \tilde{n}}^{\text {right }}=\alpha_{\tilde{n}} c_{\tilde{r} \tilde{n}}^{\text {right }}
$$

and they satisfying the conditions of completeness $c^{\text {right }} \cdot c^{\text {left }}=1$ and orthonormality $c^{\text {left }} \cdot c^{\text {right }}=1$. The coupling constants of the action

$$
S_{k}=\sum_{\tilde{n}} G_{\tilde{n}}(k) \overline{O_{\tilde{n}}^{s c}\left(\phi_{k}\right)}
$$

obviously follow (90). The operator

$$
O=\sum_{\tilde{n}} b_{\tilde{n}} \overline{\left\{O_{\tilde{n}}^{s c}\right\}_{k}},
$$

written at scale $k$ in this basis yields the parallel transport trajectory

$$
\{O\}_{k^{\prime}}=\sum_{\tilde{n}} b_{\tilde{n}}\left\{\overline{\left\{O_{\tilde{n}}^{s c}\right\}_{k}}\right\}_{k^{\prime}}=\sum_{\tilde{n}} b_{\tilde{n}}\left(\frac{k^{\prime}}{k}\right)^{\alpha_{\tilde{n}}} \overline{\left\{O_{\tilde{n}}^{s c}\right\}_{k}}
$$

in the vicinity of the fixed point.

The beta function introduced by Eqs. (72)-(74) agrees with the usual one. One finds that only relevant operators have non-vanishing parallel transport flow. Universality 
manifests itself at a given scaling regime in the suppression of the parallel transported irrelevant operators.

The blocked action $S_{k}\left[\phi_{k, x}, G_{\tilde{n}}(k), G_{\tilde{n}}(\Lambda)\right]$ possesses two interpretations:

- The value of the coupling constant at the running cut-off, $G_{\tilde{n}}(k)$, reflects the scale dependence of the physical parameters.

- The dependence on the bare coupling constants, $G_{\tilde{n}}(\Lambda)$, the initial condition of the $\mathrm{RG}$ flow provides us the generator functional for composite operators.

Finally, the composite operator renormalization represents an alternative way to arrive at the RG equation. In fact, the beta functions arising from the blocking (72) are obtained in this scheme by the parallel transport, the matching (76) of the expectation values.

\subsubsection{Perturbative treatment}

Let us finally compare the matching of the observables, described above with the traditional method of perturbative composite operator renormalization. The inversion of Eq. (77) gives

$$
\left[O_{\tilde{n}}\left(\phi_{k}+\tilde{\phi}_{k}\right)\right]_{k}=\sum_{\tilde{m}} O_{\tilde{m}}\left(\phi_{k}+\tilde{\phi}_{k}\right)\left(S^{-1}(k, \Lambda)\right)_{\tilde{m}, \tilde{n}}
$$

It is not difficult to see that the operator $\left[O_{\tilde{n}}\left(\phi_{k}+\tilde{\phi}_{k}\right)\right]_{k}$ of the bare theory which corresponds to the operator $\left\{O_{\tilde{n}}\left(\phi_{k}\right)\right\}_{k}$ of the effective theory agrees with the result of the usual composite operator renormalization. In fact, the bare action (71) can be split into the sum of the renormalized part and the counterterms the framework of the renormalized perturbation expansion, giving $G_{\tilde{n} B}=G_{\tilde{n} R}+G_{\tilde{n} C T}, G_{\tilde{n}}(\Lambda)=G_{\tilde{n} B}$ and $G_{\tilde{n}}(k)=G_{\tilde{n} R}$. The counterterms are introduced just to render certain Green functions with composite operator insertions cut-off independent [13]. The composite operator corresponding to the renormalized (ie blocked) operator $O_{\tilde{n}}\left(\phi_{k}\right)$ in this perturbative framework is

$$
\sum_{\tilde{m}} O_{\tilde{m}}\left(\phi_{k}+\tilde{\phi}_{k}\right) \frac{\delta G_{\tilde{m} B}}{\delta G_{\tilde{n} R}}
$$

which agrees with (98).

It is worthwhile recalling that the parameters and operators of a bare theory correspond to the cut-off scale in a natural manner. Therefore $\left\{O_{\tilde{n}}\left(\phi_{k}\right)\right\}_{k}$ represents the bare operator $\left[O_{\tilde{n}}\left(\phi_{k}+\tilde{\phi}_{k}\right)\right]_{k}$ in the effective description at the scale $k$ and reproduces the observational scale dependence by construction in a manner similar to the scale dependence of the hadronic structure functions obtained in the framework of the composite operator renormalization convey the same information [15].

\subsection{Continuous evolution}

The RG equations obtained so far deal with the evolution of the bare action during the gradual lowering of the U.V. cut-off. There are two different reasons to look for alternative schemes where the evolution of the Green functions rather than the bare action is followed. 
One reason is usefulness. In the traditional RG strategy followed so far the renormalized trajectories give the bare coupling constants as functions of the running cut-off. Though one can extract number of useful information from the trajectories they remain somehow qualitative because the bare parameters of the theory with the running cut-off are not physical quantities. Though this remark does not apply to the multiplicative RG scheme where the effective parameters are constructed by means of Green functions this scheme is seriously limited as mentioned in the Introduction. Returning to blocking, one can say at most that the difference between bare parameters and physical quantities arises due to the fluctuations in the path integral and this latter decreases with the number of degrees of freedom as we approach the IR end point of the trajectories. Therefore it is the blocked action with very low cut-off only which is supposed to be directly related to physical quantities (in the absence of IR instability).

Another more formal point of view is to construct a strictly non-perturbative scheme. The RG equations (10), (48) represent the complete resummation of the perturbation expansion but they are not really non-perturbative equations. This situation is reminiscent of the Schwinger-Dyson equations. They were first obtained by resumming the perturbation expansion and only later by a genuine non-perturbative method, by the infinitesimal shift of the integral variable in the path integral formalism. The fact that the naive result derived by assuming the convergence of the perturbation expansion is correct is presumably related to the unique analytic continuation of the Green functions in the coupling constants. Can we find in a similar manner the truly non-perturbative RG equations? If possible, it will come by a different route: by relating two path integral averages instead of computing them by brute force. The quantities in question are one-particle irreducible (1PI) amplitudes and we shall give up to follow the evolution of the bare coupling constants, the parameters in the path integral. This will allows us to avoid any reference to the perturbation expansion. It remains to be seen if (10) and (48) can be derived in a similar manner or they remain valid for strongly coupled models.

We begin this program by writing the generator functional for the connected Green functions in the form

$$
e^{\frac{1}{\hbar} W_{k}[j]}=\int D[\phi] e^{-\frac{1}{\hbar}\left(S_{B}[\phi]+C_{k}[\phi]-j \cdot \phi\right)},
$$

where the term $C_{k}[\phi]$ is introduced to suppress fluctuations. What we require is that (i) for $k=\infty$ the fluctuations be suppressed, $C_{\infty}[\phi]=\infty$, (ii) the original generator functional be recovered for $k=0, C_{0}[\phi]=0$ and (iii) the fluctuations are suppressed only, ie $C_{k}\left[\phi^{\prime}\right]=0$ for some configurations close to the vacuum expectation value, $\phi^{\prime} \approx\langle\phi\rangle$. The simplest choice for models without condensate is a quadratic functional,

$$
C_{k}[\phi]=\frac{1}{2} \phi \cdot C_{k} \cdot \phi
$$

but in certain cases higher order terms in the field variables are necessary in the suppression. We distinguish two kinds of suppression. The length scale of the modes 'released' 
$\ell(k)$ is well defined when

$$
\mathcal{M}_{k}^{2}(p)=\frac{\delta^{2} C_{k}}{\delta \phi_{-p} \delta \phi_{p}} \begin{cases}>>1 & |p|<1 / \ell(k), \\ \approx 0 & |p|>1 / \ell(k) .\end{cases}
$$

The evolution generated by such suppressions shows the scale dependence in the spirit of the Kadanoff-Wilson blocking, the contribution of modes with a given length scale to the dynamics. Examples are,

$$
C_{k}(p)= \begin{cases}a p^{2} \frac{f(p)}{1-f(p)} & {[16]} \\ a\left(\frac{k^{2}}{p^{2}}\right)^{b} & {[17]} \\ a\left(k^{2}-p^{2}\right) \Theta\left(k^{2}-p^{2}\right) & {[18]}\end{cases}
$$

where $f(p)=e^{-b\left(p^{2} / k^{2}\right)^{c}}$ and $a, b, c>0$. Another kind of suppression for which $\mathcal{M}_{k}^{2}(p)$ shows no clear structure will be considered in section 2.5 below.

The evolution equation for $W[j]$ is easy to obtain,

$$
\begin{aligned}
\partial_{k} W_{k}[j] & =-e^{-\frac{1}{\hbar} W_{k}[j]} \int D[\phi] \partial_{k} C_{k}[\phi] e^{-\frac{1}{\hbar}\left(C_{k}[\phi]+S_{B}[\phi]-j \cdot \phi\right)} \\
& =-e^{-\frac{1}{\hbar} W_{k}[j]} \partial_{k} C_{k}\left[\hbar \frac{\delta}{\delta j}\right] e^{\frac{1}{\hbar} W_{k}[j]} .
\end{aligned}
$$

This is already a closed functional differential equation but of little use. The reason is that it is difficult to truncate $W[j]$ being a highly non-local functional. In order to arrive at a more local functional we shall make a Legendre transformation and introduce the effective action $\Gamma_{k}[\phi]$ as

$$
\Gamma_{k}[\phi]+W_{k}[j]=j \cdot \phi, \quad \phi=\frac{\delta W[j]}{\delta j}
$$

with

$$
\partial_{k} \Gamma_{k}[\phi]=-\partial_{k} W_{k}[j]-\frac{\delta W[j]}{\delta j} \partial_{k} j+\partial_{k} j \phi=-\partial_{k} W_{k}[j] .
$$

It is advantageous to separate the auxiliary suppression term $C_{k}[\phi]$ off the effective action by the replacement $\Gamma[\phi] \rightarrow \Gamma[\phi]+C_{k}[\phi]$ resulting in

$$
\partial_{k} \Gamma_{k}[\phi]=e^{-\frac{1}{\hbar} W_{k}[j]} \partial_{k} C_{k}\left[\hbar \frac{\delta}{\delta j}\right] e^{\frac{1}{\hbar} W_{k}[j]}-\partial_{k} C_{k}[\phi] .
$$

This relation takes a particularly simple form for the quadratic suppressions (101). Since the quadratic part of the effective action is the inverse connected propagator we find [16], [10], [19], [20], [21],

$$
\partial_{k} \Gamma_{k}[\phi]=\frac{\hbar}{2} \operatorname{tr}\left[\partial_{k} C_{k} \cdot\langle\phi \phi\rangle_{\text {conn }}\right]=\frac{\hbar}{2} \operatorname{tr}\left[\partial_{k} C_{k} \cdot \frac{1}{C_{k}+\frac{\delta^{2} \Gamma_{k}}{\delta \phi \delta \phi}}\right] .
$$

Two remarks are in order in comparing the evolution equation obtained here with the pervious RG equations. First, the Kadanoff-Wilson blocking is constructed to preserve 
the generator functional $W[j]$ for the thinner system and this is realized by eliminating modes at the U.V. side, by changing $S_{k}[\phi]$. One is not aiming at keeping anything fixed in the continuous evolution scheme. The 1PI generator functional does depend on $k$ which appears as the lowest momentum of modes considered in the theory whose effective action is $\Gamma_{k}[\phi]$. The second remark is that the right hand side is proportional to $\hbar$. But the apparent absence of the mixing of tree and loop-levels is misleading since the propagator may be the sum of $\mathcal{O}\left(\hbar^{0}\right)$ tree-level and $\mathcal{O}(\hbar)$ fluctuation contributions. The explicit tree-level term is missing because the modification of the bare action was carried out in the part $\mathcal{O}\left(\phi^{2}\right)$ and this correction to the free propagator was removed by the step $\Gamma[\phi] \rightarrow \Gamma[\phi]+C_{k}[\phi]$, the substraction of the tree-level suppression term from the effective action.

It is illuminating to compare the evolution and the Schwinger-Dyson (SD) equations. Though the formal aspects appear similar the content of the equations differs. A similarity appearing immediately is that both set of equations determine the Green functions in a hierarchical manner. The derivation of the equations shows some similarity, as well. In fact, the crucial step in the derivation of the evolution equation is the first equation in (104). This step, the expression of the derivative of the functional integral formally, without its actual evaluation is the hallmark of the non-perturbative demonstration of the $\mathrm{SD}$ equations. Both the SD and the evolution equations are genuinely non-perturbative because we actually do not evaluate the functional integral, instead we relate to another one by bringing a derivative inside the path integral. In both schemes on compares two functional integrals which differ slightly, either in the infinitesimal shift of the integral variables or in the infinitesimal change in the action. Let us write the first equation in (104) for finite $\Delta k$,

$$
\begin{aligned}
& \int D[\phi]\left(e^{-\frac{1}{\hbar}\left(C_{k}[\phi]+S_{B}[\phi]-j \cdot \phi\right)}-e^{-\frac{1}{\hbar}\left(C_{k-\Delta k}[\phi]+S_{B}[\phi]-j \cdot \phi\right)}\right) \\
& =\frac{1}{\hbar} \int D[\phi]\left(\Delta k \partial_{k} C_{k}[\phi] e^{-\frac{1}{\hbar} C_{k}[\phi]}+\mathcal{O}\left((\Delta k)^{2}\right)\right) e^{-\frac{1}{\hbar}\left(S_{B}[\phi]-j \cdot \phi\right)}
\end{aligned}
$$

The small parameter $\Delta k$ is used to suppress the insertion of more field variables in the evolution equation, to cut off the higher order Green functions from the evolution, the strategy common with the non-perturbative proof of the SD equation. The higher order contributions in $\Delta k$ bring in higher order Green functions and we suppress them by the smallness of the step in 'turning on' the fluctuations. This is how the RG idea, the replacement of the higher loops by running effective coupling constants and the dealing with small number of modes at each step, is realized in the evolution equation scheme.

The obvious difference between the evolution and the SD equations is that the latter express the invariance of the functional integral in a manner similar to the RG equations but the former is simply an expression of the derivative of the functional integral with respect to a parameter. The similarity of the evolution equation and the RG strategy leads to another difference. The subsequent elimination of modes and taking into account their dynamics by the introduction/modification of effective vertices generates a 'universal' evolution equations which does not depend on the theory in question. In fact, the 
evolution equation (108) and the functional RG equations Eqs. (6), (45) do not contain any reference to the model considered. The effective or the bare action of the model appear in the initial condition only. The SD equation is based on a careful application of the equation of motion within the expectation values and therefore contains the action of the model in an obvious manner.

The evolution equation method seems to be better suited to numerical approximations then the SD equations. This is because the 'dressing', the summing up the interactions is achieved by integrating out differential equations rather then coupling the Green functions in the SD hierarchy, a numerical problem we can control easier.

\subsection{Blocking in the internal space}

We present now a generalization the RG method. The traditional RG strategy is aiming at the scale dependence of observables and consequently follows the cut-off dependence in the theories. The results are obtained by the successive modification of the cut-off and the accumulation of the resulting effects. One can generalize this method by replacing the cut-off by any continuous parameters of the theory on which the observables depend upon in a differentiable manner. Such a generalization replaces the RG equation by an evolution equation corresponding to the parameter or coupling constant in question.

One gains and looses in the same time during such a generalization. One looses intuition of and insight into the dynamics since the trajectories generated by the evolution equation have no relation to scale dependence. To make things even more complicated the trajectories do not correspond anymore to a fixed physical content, instead they trace the dependence of the dynamics in the parameter considered. But we gain in flexibility. In fact, the parameter we select to evolve can be the Plank constant or a coupling constant and the integration of the resulting evolution resums the semiclassical or the perturbation expansion. We may apply this method in models with non-trivial saddle point structure or where the cut-off would break important symmetries, e.g. in gauge theories.

A more technical aspect of this generalization touches the U.V. divergences in the theory. The traditional RG procedure is based on the tacit assumption that the U.V. divergences are properly regulated by the blocking, the moving cut-off. This seems to be a natural requirement if the RG flow is interpreted as scale dependence. In fact, an U.V. divergence left-over by the blocking would indicate the importance of the processes at the regulator and would lead to the appearance of a second scale. In the generalization of the RG procedure we introduce below there may not be a well defined scale related to the evolution and we have to regulate the U.V. divergences.

There are two different kind of physical spaces in Field Theory. The space-time or the momentum-energy space where the 'events' are taking place will be called external space. The 'events' are characterized by further quantities, the field amplitudes. The space where the field amplitudes belong will be called internal space. Therefore the field configuration $\phi_{x}$ realizes a map $\phi:$ external space $\rightarrow$ internal space. The traditional Kadanoff-Wilson blocking orders the degrees of freedom to be eliminated according to 
their scale in the external space. We may realize a similar scheme by ordering the modes according to their scale in the internal space, their amplitude [22]. We present two different implementations of this idea, one for Wilsonian and another for the effective action.

\subsubsection{Wilsonian action}

We shall use the flexibility of the method of deriving the Polchinksi equation in section 2.2 to separate off and eliminate the fluctuation modes with the largest amplitude in the path integral. Since the fluctuation amplitude is controlled by the mass this leads to the description of the dynamics by highly massive modes, small fluctuations at the final point of the evolution.

Let us consider the model (38) where the role of the parameter $k$ is played by the mass $M$ and $G_{M^{2}}^{-1}=p^{2}+M^{2}$. We shall follow the evolution from $M^{2}=0$ to $M^{2}>>\Lambda^{2}$ where $\Lambda$ is the U.V. cut-off. The decomposition

$$
G_{M^{2}}=G_{M^{2}+\Delta M^{2}}+\tilde{G}_{M^{2}}, \quad \Phi=\phi+\tilde{\phi},
$$

will be used with

$$
\frac{1}{2} \phi\left(M^{2}+\Delta M^{2}-\square\right) \phi+\frac{1}{2} \tilde{\phi} \frac{\left(M^{2}-\square\right)^{2}}{\Delta M^{2}} \tilde{\phi}=\frac{1}{2} \Phi\left(M^{2}-\square\right) \Phi+\frac{1}{2} \tilde{\Phi} \tilde{G}_{D}^{-1} \tilde{\Phi} .
$$

We replace the degrees of freedom $\tilde{\Phi}$ by the field $\tilde{\phi}$ with infinitesimal fluctuations. After eliminating $\tilde{\phi}$ the remaining field $\phi$ has smaller fluctuations than $\Phi$. We use the ansatz

$$
S_{M}^{I}[\phi]=\int_{x}\left[\frac{1}{2}\left(Z_{M}\left(\phi_{x}\right)-1\right) \partial_{\mu} \phi_{x} \partial_{\mu} \phi_{x}+U_{M}\left(\phi_{x}\right)\right]
$$

where the U.V. regulator with smooth cut-off is not shown explicitly. We follow the steps outlined in section 2.2.2 which gives rise the evolution equation with left hand side

$$
\partial_{M^{2}}\left\{\frac{1}{2} \int_{p} \eta_{-p} \eta_{p}\left[(Z-1) p^{2}+U^{(2)}\right]+V U\right\} .
$$

The right hand side reads as

$$
\begin{aligned}
& -\frac{1}{2} \int_{p}\left\{\delta_{p, 0} U^{(1)}+\eta_{p}\left[(Z-1) p^{2}+U^{(2)}\right]+\frac{1}{2} \int_{r} \eta_{r} \eta_{p-r}\left[U^{(3)}-Z^{(1)}\left(r \cdot p-r^{2}+p^{2}\right)\right]\right\} \\
& \times \frac{1}{\left(p^{2}+M^{2}\right)^{2}} \\
& \times\left\{\delta_{p, 0} U^{(1)}+\eta_{-p}\left[(Z-1) p^{2}+U^{(2)}\right]+\frac{1}{2} \int_{s} \eta_{-p-s} \eta_{s}\left[U^{(3)}+Z^{(1)}\left(s^{2}+p \cdot s+p^{2}\right)\right]\right\} \\
& +\frac{\hbar}{2} \int_{p} \frac{1}{\left(p^{2}+M^{2}\right)^{2}}\left\{V\left[(Z-1) p^{2}+U^{(2)}\right]+\frac{1}{2} \int_{r} \eta_{-r} \eta_{r}\left[U^{(4)}+Z^{(2)}\left(p^{2}+r^{2}\right)\right]\right\} \\
& \approx-V U^{(1) 2} \frac{1}{2 M^{4}}-\frac{1}{2} \int_{r} \eta_{-r} \eta_{r}\left\{\frac{\left[(Z-1) r^{2}+U^{(2)}\right]^{2}}{\left(r^{2}+M^{2}\right)^{2}}+\frac{1}{M^{4}} U^{(1)}\left(U^{(3)}+Z^{(1)} r^{2}\right)\right\} \\
& +\frac{\hbar}{2} \int_{p} \frac{1}{\left(p^{2}+M^{2}\right)^{2}}\left\{V\left[(Z-1) p^{2}+U^{(2)}\right]+\frac{1}{2} \int_{r} \eta_{-r} \eta_{r}\left[U^{(4)}+Z^{(2)}\left(p^{2}+r^{2}\right)\right]\right\}
\end{aligned}
$$


The system of evolution equation projected onto the different $\eta$-dependent terms is

$$
\begin{aligned}
2 M^{4} \partial_{M^{2}} U & =-U^{(1) 2}+\hbar M^{4} \int_{p} \frac{(Z-1) p^{2}+U^{(2)}}{\left(p^{2}+M^{2}\right)^{2}} \\
2 M^{4} \partial_{M^{2}} Z & =-4 U^{(2)}(Z-1)+4 \frac{U^{(2) 2}}{M^{2}}-2 U^{(1)} Z^{(1)}+\hbar M^{4} Z^{(2)} \int_{p} \frac{1}{\left(p^{2}+M^{2}\right)^{2}} \\
2 M^{4} \partial_{M^{2}} U^{(2)} & =-U^{(2) 2}-U^{(1)} U^{(3)}+\hbar M^{4} \int_{p} \frac{Z^{(2)} p^{2}+U^{(4)}}{\left(p^{2}+M^{2}\right)^{2}} .
\end{aligned}
$$

The $\mathcal{O}\left(\hbar^{0}\right)$ terms on the right hand side are to keep the tree-level observables $M$-invariant within the gradient expansion ansatz. As far as the loop corrections are concerned, the beta functions

$$
\beta_{n}=M \partial_{M} g_{n}=\hbar M^{2} U^{(n+2)} \int_{p} \frac{1}{\left(p^{2}+M^{2}\right)^{2}},
$$

obtained from the first equation with $Z=1$ agree with the leading order contribution to the one-loop renormalized potential,

$$
U_{M}^{1-\text { loop }}=U_{M}+\frac{\hbar}{2} \int_{p} \ln \left[p^{2}+M^{2}+U_{M}^{(2)}\right]
$$

except their sign. This is because we intend to keep the partition function unchanged as opposed to Eq. (117) where the mass is evolving together with the dynamics.

\subsubsection{Effective action}

Let us consider for the sake of simplicity again the scalar model given by the bare action

$$
S_{B}=\int_{x}\left[\frac{1}{2}\left(\partial_{\mu} \phi_{x}\right)^{2}+\frac{m_{B}^{2}}{2} \phi_{x}^{2}+U_{B}\left(\phi_{x}\right)\right]
$$

and the suppression with no structure in the external space [22],

$$
C_{k}(p)=\frac{M^{2}}{2}
$$

which acts as a 'smooth cut-off' for the amplitude of the fluctuations. The corresponding evolution equation is

$$
\partial_{M^{2}} \Gamma_{M}[\phi]=\frac{\hbar}{2} \operatorname{tr}\left[M^{2}+\frac{\delta^{2} \Gamma_{M}[\phi]}{\delta \phi \delta \phi}\right]^{-1} .
$$

The effective action of the theory is obtained by integrating this equation from the initial condition $\Gamma_{M_{0}}[\phi]=S_{B}[\phi]$ imposed at $M^{2}=M_{0}^{2}>>\Lambda^{2}>>m_{B}^{2}$ to $M=0$.

Let us consider how this scheme looks like for the ansatz

$$
\Gamma_{M}[\phi]=\int_{x}\left[\frac{1}{2} Z_{M}\left(\phi_{x}\right)\left(\partial_{\mu} \phi_{x}\right)^{2}+U_{M}\left(\phi_{x}\right)\right] .
$$


One finds

$$
\begin{aligned}
\partial_{M^{2}} U= & \frac{\hbar}{2} \int_{p} \frac{1}{Z p^{2}+M^{2}+U^{(2)}} \\
\partial_{M^{2}} Z= & \frac{\hbar}{2} \int_{p}\left[2 Z^{(1)} \frac{\frac{p^{2}}{d} Z^{(1)}+2\left(Z^{(1)} p^{2}+U^{(3)}\right)}{\left(Z p^{2}+M^{2}+U^{(2)}\right)^{3}}-\frac{Z^{(2)}}{\left(Z p^{2}+M^{2}+U^{(2)}\right)^{2}}\right. \\
& -2 Z \frac{\left(Z^{(1)} p^{2}+U^{(3)}\right)^{2}}{\left(Z p^{2}+M^{2}+U^{(2)}\right)^{4}}-\frac{8 p^{2}}{d} Z Z^{(1)} \frac{\left(Z^{(1)} p^{2}+U^{(3)}\right)}{\left(Z p^{2}+M^{2}+U^{(2)}\right)^{4}} \\
& \left.+\frac{8 p^{2}}{d} Z^{2} \frac{\left(Z^{(1)} p^{2}+U^{(3)}\right)^{2}}{\left(Z p^{2}+M^{2}+U^{(2)}\right)^{5}}\right] .
\end{aligned}
$$

The asymptotic form of the first equation for $M^{2}>>U^{(2)}$ in the local potential approximation,

$$
M \partial_{M} U_{M} \approx-U_{M}^{(2)} \int_{p} \frac{M^{2}}{\left(p^{2}+M^{2}\right)^{2}}
$$

up to field independent constant and it agrees with asymptotic form of the first equation in (115) except the sign. The integrand in (123) corresponds to transformation rule (4) of the propagator under the infinitesimal change of the mass for the first, leading order graphs in Figs. 3. The numerical integration resums the higher orders in the loop expansion. In short, we see the Callan-Symanzik scheme at work in the functional formalism.

The suppression (119) freezes out modes with momentum $p$ if $p^{2}<M^{2}$ and generates a characteristic scale $p_{\text {cr }}$ where $\left(Z p^{2}+M^{2}+U^{(2)}\right) /\left(Z p^{2}+U^{(2)}\right)$ deviates from 1 . So long this scale is far from the intrinsic scales of the model, $M^{2}>>U^{(2)}$ in the U.V. scaling regime, such an internal space blocking generates the external scale $p_{\text {cr }} \approx M / \sqrt{Z}$. In other words, the universal part of the beta functions obtained in the U.V. regime by means of the Callan-Symanzik scheme should agree with the same part of the beta functions coming from other schemes. This agreement has been observed in the framework of the scalar model [23]. We shall check this quickly in the local potential approximation. By comparing Eq. (123) with the asymptotic form of the WH equation (14),

$$
k \partial_{k} U_{k}=-\frac{\hbar U_{k}^{(2)} \Omega_{d} k^{d-2}}{2(2 \pi)^{d}},
$$

we find

$$
\frac{d k^{2}}{d M^{2}}=2 \int_{0}^{\Lambda / k} \frac{y^{d-1} d y}{\left(y^{2}+M^{2} / k^{2}\right)^{2}} .
$$

Below the upper critical dimension, $d<4$, the right hand side is finite and the external (WH) and the internal (CS) scales are proportional. At the critical dimension the $M$ dependence of the right hand side is through the proportionality factor $\ln \left(1+\Lambda^{2} / M^{2}\right)$. The two scales become approximately proportional only for much higher value of the cut-off $\Lambda$, since the RG flow spends more 'time' close to the non-universal short distance regime due to the tree-level marginality of $g_{4}$. There is no agreement beyond $d=4$ where 
the cut-off scale is always important. The agreement between the two schemes is violated by higher order terms in the perturbation expansion since they represent the insertion of irrelevant effective vertices and by leaving the asymptotical U.V. regimes. This explains that similar argument does not hold when $Z$ displays important $M$-dependence.

It is worthwhile noting that the non-trivial wave function renormalization $Z$ and the corresponding anomalous dimension $\eta$ of the field variable can be thought as the reflection of the mismatch between the scale dependence in the external and internal spaces around the U.V. fixed point. In fact, the phenomenological form

$$
\left\langle\phi_{x} \phi_{y}\right\rangle \approx c|x-y|^{2-d-\eta}
$$

cf Eq. (128) below connects the fundamental dimensional objects of the internal and external spaces.

The flexibility of choosing the suppression functional $C_{k}[\phi]$ may be important for certain models. By choosing

$$
C_{k}[\phi]=\frac{k}{\Lambda} S[\phi]
$$

where $\Lambda$ is the U.V. cut-off the evolution in $k$ resums the loop expansion since the effective Planck-constant, $\hbar^{-1}(k)=\hbar^{-1}+k / \Lambda$ evolves from 0 to $\hbar$. This scheme is advantageous for models with inhomogeneous saddle point eg solitons or instantons because their spacetime structure is 'RG invariant', being independent of the gradual control of the amplitude of the fluctuations. Another advantage offered by the flexibility in choosing the suppression functional is the possibility of preserving symmetries, the point considered in section 3.5 below.

\section{Applications}

We shall briefly review a few applications of the functional evolution equations. An incomplete list of the developments not followed due to limitation in time is the following. The exciting competition for the 'best' critical exponents have led to several works using this method [24], [53], [17], [50], [51], [25], [57], [26]. Such kind of application opens up the issue of understanding the impact of truncation on the blocking [27], [28], [29] [25], [30], [31] and looking for optimization [32], [33], [18]. The phase structure and the nature of phase transitions are natural subjects [21], [34], [35]. The incorporation of fermions is essential to arrive at realistic models in High Energy [36] and Condensed Matter Physics [37]. The computation of the quenched average of Green functions, one of the essential obstacle of progress in Condensed Matter Physics can be approached in a new fashion by applying the internal space renormalization group method [38]. A promising application is in general relativity [39]. Finally, the infamous problem of bound states can be reconsidered in this framework [40], [41].

Much more to be found in review articles [42], conference proceedings [43] and $\mathrm{PhD}$ thesis [44]. 


\subsection{Fixed points}

\subsubsection{Rescaling}

'The original form of the RG procedure consists of two steps, the blocking, followed by a rescaling. The latter can be omitted if the RG strategy is used to solve models only. But it becomes important when we try to understand the scale dependence of the theories. Our physical intuition is based on classical physics and particles. After losing much of the substance during the quantization procedure there is a chance to recover some clarity by the introduction of quasiparticles, localized excitations with weak residual interaction. The rescaling step of the $R G$ scheme is to check a given quasiparticle assumption by removing the corresponding scale dependence. The remaining scale dependence is a measure of the quality of the quasiparticle picture. The deviation from this non-interactive system is parametrized in terms of anomalous dimensions.

The particle content of a theory is usually fixed by the quadratic part of its action. Therefore the rescaling is defined in such a manner that this part of the action stays invariant during the blocking. For an $O(d)$ invariant Euclidean or relativistically invariant real-time system the resulting rescaling reflects the classical dimension of the dynamical variables and coupling constants. For non-relativistic systems the fixed point scaling is an artificial device only to identify the non-interacting part of the action. When smooth cut-off is used its details may influence of the scaling properties of the quadratic action, as well, and may induce deviations from classical dimensions..

Consider the scale transformation

$$
p \rightarrow p^{\prime}=(1+\epsilon) p, \quad x \rightarrow x^{\prime}=(1-\epsilon) x, \quad \phi\left((1+\epsilon) x^{\prime}\right) \rightarrow\left(1-\epsilon d_{\phi}\right) \phi(x)
$$

in an $O(d)$ invariant scalar model where $d_{\phi}=(d-2+\eta) / 2$. The parameter $\eta$ reflects the deviation of from classical dimensional analysis. The effect of this rescaling on the action

$$
S[\phi]=\sum_{n} \int_{p_{1}, \cdots, p_{n}} u_{p_{1}, \cdots, p_{n}} \delta_{p_{1}+\cdots+p_{n}, 0} \phi_{p_{1}} \cdots \phi_{p_{n}},
$$

can be found in the following manner [45]:

- The momentum integral measure changes as $d^{d} p \rightarrow(1-d \epsilon) d^{d} p^{\prime}$, giving $S \rightarrow[1-$ $\left.\epsilon d \int_{p} \phi_{p} \frac{\delta}{\delta \phi_{p}}\right] S$.

- The coupling constants change as $u_{p_{1}, \cdots, p_{n}} \rightarrow u_{(1-\epsilon) p_{1}^{\prime}, \cdots,(1-\epsilon) p_{n}^{\prime}}$. This can be written as the transformation $S \rightarrow\left[1-\epsilon \int_{p} \phi_{p} p \cdot \partial_{p}^{\prime} \frac{\delta}{\delta \phi_{p}}\right] S$ of the action where the prime on the gradient $\partial_{p}$ indicates that the derivative acts on the coupling constants only and not on the Dirac-deltas.

- The Dirac-deltas change as $\delta_{p_{1}+\cdots+p_{n}, 0} \rightarrow \delta_{(1-\epsilon) p_{1}^{\prime}+\cdots+(1-\epsilon) p_{n}^{\prime}, 0}$, amounting to the transformation $S \rightarrow[1+d \epsilon] S$ of the action.

- Finally, the field transforms as $\phi_{p} \rightarrow\left[1-\epsilon\left(d_{\phi}-d\right)\right] \phi_{p}$, inducing $S \rightarrow\left[1-\epsilon\left(d_{\phi}-\right.\right.$ d)] $\int_{p} \phi_{p} \frac{\delta}{\delta \phi_{p}} S$.

Adding up these contributions we find the rescaling generator

$$
\mathcal{G}=-\int_{p}\left[\phi_{p} p \cdot \partial_{p}^{\prime} \frac{\delta}{\delta \phi_{p}}+d_{\phi} \phi_{p} \frac{\delta}{\delta \phi_{p}}\right]
$$


The $R G$ equation for the rescaled action can be obtained by adding $\mathcal{G}$ acting on the (effective) action to the right hand side, eg the evolution (49) turns out to be

$$
k \partial_{k} e^{-\frac{1}{\hbar} S_{k}^{I}[\phi]}=(\mathcal{G}+\hbar \mathcal{B}) e^{-\frac{1}{\hbar} S_{k}^{I}[\phi]} .
$$

The rescaling of the field may be viewed as a transformation of the action without changing the physics at the fixed point. The terms generated on the fixed point action by the rescaling, $\mathcal{G} S^{*}[\phi]$ are called redundant at the fixed point in question.

\subsubsection{Reparametrizing}

We considered linear rescaling of the field variable but one can easily generalize the rescaling to non-linear reparametrization. The infinitesimal change $\phi_{x} \rightarrow \phi_{x}^{\prime}=\phi_{x}+$ $\epsilon \Psi[\phi ; x]$ performed inside the path integral gives

$$
\int D[\phi] e^{-S[\phi]} \rightarrow \int D\left[\phi^{\prime}\right] e^{-S\left[\phi^{\prime}\right]}=\int D[\phi]\left[1+\epsilon \int_{x} \frac{\delta \Psi[\phi ; x]}{\delta \phi_{x}}\right] e^{-S[\phi]-\epsilon \int_{x} \Psi[\phi ; x] \frac{\delta S[\phi]}{\delta \phi_{x}}}
$$

The reparametrization invariance of the integral assures that the modification

$$
S[\phi] \rightarrow S[\phi]+\epsilon \mathcal{G}_{\Psi} S[\phi]
$$

of the action where

$$
\mathcal{G}_{\Psi}=\int_{x}\left[\Psi[\phi ; x] \frac{\delta S[\phi]}{\delta \phi_{x}}-\frac{\delta \Psi[\phi ; x]}{\delta \phi_{x}}\right]
$$

is an invariance of the partition function. It has furthermore been noted [46] that some of the infinitesimal blocking relations for the (effective) action can be written in the form (133).

Though it is certainly very interesting to find a common structure for the functional $\mathrm{RG}$ equations the connection with reparametrization invariance is not clear. The point is that non-linear reparametrizations can not be carried out inside of the path integral as in ordinary integrals. The problem arises from the fact that the typical configurations are rather singular in the path integration. Calculus known from classical analysis is replaced by Ito-calculus in Quantum Mechanics due to the nowhere-differentiable nature of the quantum trajectories [47]. The quantum field configurations are even more singular. Consider a free massless field $\phi$ in $d$ dimensions and its partition function

$$
Z=\prod_{x} \int d \phi_{x} e^{-\frac{a^{d-2}}{2} \sum_{x}\left(\Delta_{\mu} \phi_{x}\right)^{2}}=\prod_{x} \int d \phi_{x} e^{-\frac{1}{2} \sum_{x}\left(\Delta_{\mu} \tilde{\phi}_{x}\right)^{2}}
$$

where $\Delta_{\mu} \phi_{x}=\phi_{x}-\phi_{x-\mu}$ and the dimension of the field was removed in the second equation, $\tilde{\phi}=a^{d / 2-1} \phi$. The typical configurations have $\Delta \tilde{\phi}=\mathcal{O}\left(a^{0}\right)$, ie the discontinuity of the original field variable is $\Delta \phi=\mathcal{O}\left(a^{1-d / 2}\right)$.

This simple scaling, the basis of the usual U.V. divergences in quantum field theory, gives non-differentiability in Quantum Mechanics, for $d=1$, finite discontinuity in $d=2$ and diverging discontinuities for $d>2$. The non-differentiability can be represented 
by effective potential vertices in Quantum Mechanics, [48], a reflection of the unusual quantization rules in polar coordinates, sensitivity for operator ordering and quantum anomalies [49]. In two dimensions the discontinuity is finite and the continuous structure can either be preserved or destroyed by quantum fluctuations, cf. section 3.4. In higher dimensions the singularities remain always present. It is this singular nature of the trajectories which requires that one goes to unusually high order in the small parameter $\epsilon$ characterizing the infinitesimal change of variables in the path integral. This problem, composite operator renormalization, renders the non-linear change of variables a poorly controlled subject in quantum field theories.

\subsubsection{Local potential approximation}

We shall search for the fixed points of the Polchinski equation in the local potential approximation, [50] [51]. We introduce dimensionless quantities $\phi=k^{1-d / 2} \Phi$ and $u_{k}(\phi)=$ $k^{d} U_{k}(\Phi)$. The RG equation with rescaling for $u$ is

$$
\dot{u}=-d u+\frac{\eta+d-2}{2} \phi u^{(1)}-u^{(1) 2} K_{0}^{\prime}+\hbar \bar{K}^{\prime} u^{(2)}
$$

where $\bar{K}^{\prime}=k^{-d} \int_{p} K_{p}^{\prime}$ and we set $\eta=0$ in the approximation $Z=1$. Notice the explicit dependence on the cut-off function $K$. This reflects the fact that the RG flow depends on the choice blocking transformation. Only the qualitative, topological features of the renormalized trajectory and the critical exponents around a fixed point are invariant under non-singular redefinitions of the action.

Gaussian fixed point: The fixed point equation, $\dot{u}^{*}=0$ has two trivial solutions,

$$
u^{*}(\phi)=0
$$

and

$$
u^{*}(\phi)=\frac{\phi^{2}}{2\left|K_{0}^{\prime}\right|}+\hbar \frac{\bar{K}^{\prime}}{d K_{0}^{\prime}}
$$

where $K_{0}^{\prime}, \bar{K}^{\prime}<0$. For any other solution $u=\mathcal{O}\left(\phi^{2}\right)$ as $\phi \rightarrow \infty$.

In order to find the scaling operators we introduce a perturbation around the fixed point by writing $u=u^{*}+\epsilon k^{-\lambda} v(\phi)$ where $\epsilon$ is infinitesimal and solve the linearized eigenvalue equation

$$
\hbar \bar{K}^{\prime} v^{(2)}=(d-\lambda) v+\left(\frac{2-d}{2} \phi+2 u^{*(1)} K_{0}^{\prime}\right) v^{(1)} .
$$

Having a second order differential equation one can construct a one-parameter family of solution after having imposed say $v^{(1)}(0)=1$ in a theory with the symmetry $\phi \rightarrow-\phi$. But the polynomial solutions

$$
v_{n}(\phi)=\sum_{\ell=1}^{n} v_{n, \ell} \phi^{2 \ell}
$$

which are parametrized by a discrete index $n$ correspond to discrete spectrum [52]. The critical exponents are identified by comparing the terms $\mathcal{O}\left(\phi^{2 n}\right)$ in Eq. (139), $\lambda_{n}=$ 
$d+(2-d) n$, and $\lambda_{n}=d-(d+2) n$, for the fixed points (137) and (138), respectively [53]. The leading order critical exponents are given entirely by the tree-level contributions. The dimensionful coupling constants are cut-off independent in this case and we have $\lambda_{n}=\left[g_{2 n}\right]$ for the fixed point (137) where the dimension of the coupling constant $[g]$ is given by Eq. (24). The dominant, largest exponent $\lambda_{T}$ and the corresponding coupling constant play distinguished role in the scaling laws. The dimensionless coupling constant can be identified with the 'reduced temperature' $t$. According to the definition $\xi \approx t^{-\nu}$ of the critical exponent $\nu$ we have the mean-field exponent, $\nu=1 / \lambda_{T}=1 / 2$. The mass is relevant at the point (137) as expected, as shown by the beta function $\beta_{2}=-2 K_{0}^{\prime} \tilde{g}_{2}^{2}-2 \tilde{g}_{2}$, according to Eq. (55). The scaling potentials with exponential growth for large $\phi[54]$ correspond to the continuous spectrum and their physical interpretation is unclear.

All exponents are negative at (138), this is an IR fixed point.

Wilson-Fischer fixed point: There are non-Gaussian fixed point solutions for Eq. (136) when $2 \leq d<4$. One can construct a one-parameter family of solutions but the fixed point potentials which remain non-singular for arbitrary $\phi$ correspond to a discrete set [55], [52]: the Wilson-Fischer fixed point for $3<d<4$ and as many fixed points as relevant when $2<d<3$. The perturbation around these fixed points is a oneparameter family of scaling potentials with continuous spectrum of critical exponents. The restriction for solution which are finite and non-singular everywhere produces a discrete spectrum [56] in good agreement with other methods of determining the critical exponents [53], [57].

The truncation of the fixed point solution at a finite order of $\phi$ introduces error and spurious solutions [27], [28] which can partially be eliminated by expanding the potential along the cut-off dependent minimum [29].

\subsubsection{Anomalous dimension}

The RG equation for the wave function renormalization constant $z(\phi)=Z(\Phi)$ is

$$
\begin{aligned}
\dot{z}= & -4 K_{0}^{\prime} u^{(2)}(z-1)-2 K_{0}^{\prime} u^{(1)} z^{(1)}-2 \frac{K_{0}^{\prime \prime} K_{0}}{k^{2}} u^{(2) 2} \\
& +\hbar z^{(2)} \bar{K}^{\prime}-\eta(z-1)+\frac{d+\eta-2}{2} \phi z^{(1)} .
\end{aligned}
$$

The lesson of the case $Z=1$ is that the requirement of the existence and finiteness of $u^{*}(\phi)$ introduces discrete number of fixed point solutions. Let us try to follow the same strategy again. The dominant terms of such fixed point solutions $\dot{u}=\dot{z}=0$ of Eqs. (136) and (141) are

$$
u^{*}(\phi) \approx \frac{2-\eta}{4} \phi^{4}+A \phi^{\frac{d-2+\eta}{d+2-\eta}}, \quad z^{*}(\phi) \approx B .
$$

Together with the conditions $u^{(1)}(0)=z^{(1)}(0)=0$ imposed for the $\phi \rightarrow-\phi$ symmetrical models the solution are well determined in terms of $A$ and $B$. The problem is that such kind of argument does not fix the value of $\eta$.

There is another condition to be fulfilled by the fixed points, the critical exponents should be invariant under rescaling. This is sufficient to determine $\eta$. Unfortunately the 
rescaling invariance of the fixed point is lost unless sharp or specially chosen polynomial smooth cut-off is used [56], [58]. Furthermore the truncation of the gradient expansion contributes to the violation of this invariance, too. An approximation to find the 'best' solution when the rescaling invariance is not respected is the following [51]: Introduce a further condition which violates rescaling invariance, say fix the value of $z(0)$. This allows the determination of $\eta$ which would be unique if rescaling could be used to relax our last condition. We are as close as possible to the invariant situation within our parametrized problem when the dependence of $\eta$ on $z(0)$ is the slowest. Therefore the condition $d \eta / d z(0)=0$ selects the 'best' estimate of $\eta$.

The numerical error due to the truncation of the gradient expansion has been the subject of extensive studies in the framework of the scalar model, c.f. Ref. [42]. The general trend is that the truncation of the gradient expansion at the local potential approximation (zeroth-order) or at the wave function renormalization constant level (first order) yields approximately $10 \%$ or $3 \%$ difference in the critical exponents compared with Monte-Carlo simulations, seven-loop computations in fixed dimensions or the fifth-order results of the expilon-espansion.

\subsection{Global RG}

The usual application of the RG method can be called local because as in the determination of the critical exponent it is performed around a point in the space of coupling constants. Models with more than one scale may visit several scaling regimes as the observational scale changes between the U.V. and the IR fixed points. The determination of the 'important' coupling constants of such models which parametrize the physics goes beyond the one-by-one, local analysis of the scaling regimes. It requires the careful study of crossovers, the overlap between the scaling laws of different fixed points, a problem considered in this section.

\subsubsection{RG in Statistical and High Energy Physics}

It is important to realize the similarity the way RG is used in Statistical and High Energy Physics despite the superficial differences. The most obvious difference is that while the running coupling constants are introduced in Particle Physics by means of Green functions or scattering amplitudes at a certain scale, the parameters of Solid State Physics models are defined at the cut-off, the latter being a finite scale parameter, eg lattice spacing. Since the bare coupling constants characterize the strength of the physical processes at the scale of the cut-off, the two ways of defining the scale dependence are qualitatively similar and reproduce the same universal scaling laws.

The U.V. fixed point where the correlation length diverges in units of the lattice spacing in Statistical Mechanics corresponds to renormalized theory in High Energy Physics where the U.V. cut-off is removed.

There are two classification schemes of operators, one comes from Statistical and the other from High Energy Physics: if the coupling constant of an operator increases, stays 
constant or decreases along the renormalized trajectory towards the IR the operator is called relevant, marginal or irrelevant in Statistical Physics. The coupling constants which can be renormalized as the U.V. cut-off is removed in such a manner that observables converge are called renormalizable in High Energy Physics. The important point is these classification schemes are equivalent, in particular the set of irrelevant operators at an U.V. fixed point agrees with the set of non-renormalized ones.

One can easily give a simple quantitative argument in the leading order of the perturbation expansion. First let us establish the power counting argument about renormalizability. Suppose for the sake of simplicity that there is only one coupling constant, $g$, playing the role of small parameter and a physical quantity is obtained as

$$
\langle\mathcal{O}\rangle=\sum_{n} g^{n} I_{n}
$$

where $I_{n}$ is sum of loop integrals. This series gives rise the expression

$$
\left[I_{n}\right]=[\mathcal{O}]-n[g]
$$

for the (mass) dimension of the loop integral. Let us recall that the degree of the overall (U.V.) divergence of a loop integral is given by its dimension. We distinguish the following cases:

- $[g]<0$ : The higher powers of $g$ decrease the dimension in (143) which is compensated for by increasing the degree of the overall divergence of the loop integrals, cf Eq. (144). Graphs with arbitrary high degree of divergence appear in the perturbation expansion and $g$ is called non-renormalizable.

- $g[g]>0$ : The higher order loop integrals are less divergent, there are finite number of divergent graphs in the perturbation series of any observable. The coupling constant is super-renormalizable.

- $[g]=0$ : The maximal degree of (U.V.) divergence is finite for any observable but there are infinitely many U.V. divergent graphs. $g$ is a renormalizable coupling constants.

Since the IR degree of divergence of massless loop integrals is just $-\left[I_{n}\right]$ the perturbation expansion of a super-renormalizable or non-renormalizable massless model is IR unstable (divergent) or stable (finite), respectively. The compromise between the U.V. and the IR behaviors is attained by dimensionless, renormalizable coupling constants. The IR stability can be realized by a partial resummation of the perturbation expansion in massless super-renormalizable models [59].

It is rather cumbersome but possible to prove by induction that the definition of renormalizability outlined above according to the overall divergence of the loop integrals remains valid in every order of the perturbation expansion.

In order to separate off the trivial scale dependence one usually removes the classical dimension of the coupling constants by means of the cut-off in Statistical Physics. On the tree-level, in the absence of fluctuations classical dimensional analysis applies giving the relation $g=k^{[g]} \tilde{g}$ between the dimensional and dimensionless coupling constants, $g$ 


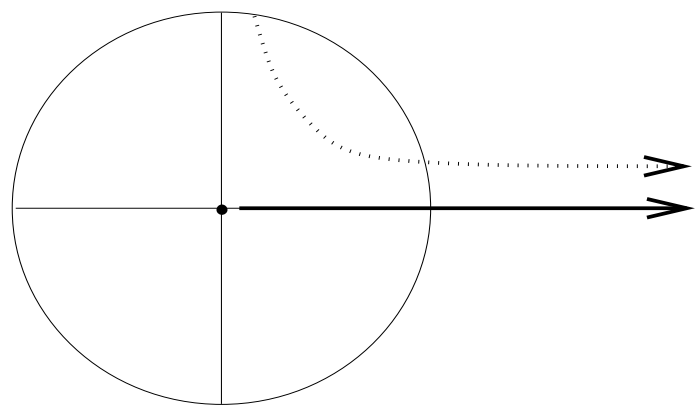

Fig. 5 An U.V. fixed point and its vicinity. The $x$ and the $y$ axis correspond a relevant and an irrelevant operator, respectively. The circle denotes the vicinity of the fixed point where the blocking relation is linearizable.

and $\tilde{g}$, respectively. Assuming that there is no evolution on the tree-level we have the scaling law

$$
\tilde{g}(k)=\left(\frac{k}{\Lambda}\right)^{-[g]+\mathcal{O}(\hbar)} \tilde{g}(\Lambda)
$$

for the dimensionless coupling constants, showing that the non-renormalizability of a coupling constant, $[g]<0$ is indeed equivalent to irrelevance, the decrease of the coupling constant towards the IR direction. Higher loop contributions do not change this conclusion so long as the loop expansion is convergent and the anomalous dimension, the contribution $\mathcal{O}(\hbar)$ in the exponent can not overturn the sign of the classical dimension $[g]$. In case of a marginal coupling constant, $[g]=0$ on has to carry on with the loop expansion until the first non-vanishing contribution to the anomalous dimension.

One can construct a much simpler and powerful argument for the equivalence of the irrelevant and the non-renormalizable set of coupling constants in the following manner. Consider the U.V. fixed point $P$ and the region around it where the blocking relations can be diagonalized, as depicted in Fig. 5. The solid line shows a renormalized trajectory of a model which contains relevant operator only at the cut-off. The Lagrangian of another model whose trajectory is shown by the dotted line contains an irrelevant operator, as well. The difference between the two models becomes small as we move towards the IR direction and the physics around the end of the U.V. scaling regime is more independent on the initial value of the irrelevant coupling constants ${ }^{\ddagger}$ longer the U.V. regime is. This is what is called universality of the long range, low energy phenomena. By looking "backwards" and increasing the cut-off energy, as done in Particle Physics, the nonvanishing irrelevant coupling constants explode and the trajectory is deflected from the fixed point. As a result we cannot maneuver ourselves into the U.V. fixed point in the presence of irrelevant operators in the theory. Since the infinite value of the cut-off corresponds to the renormalized theory, represented by the fixed point, the irrelevant operators are non-renormalizable. Despite its simplicity, this argument is valid without invoking any small parameter to use in the construction of the perturbation expansion.

Renormalizable models were thought to be essential in High Energy Physics. These models in which a sufficiently large class of observables can be made convergent are

\footnotetext{
$\varsubsetneqq$ More precisely all the irrelevant coupling constants can modify is an overall scale.
} 
distinguished by their simplicity, their interactions exist without any cut-off. Examples are Yang-Mills models in four dimensions and the sine-Gordon, Thirring and the X-Y models in two dimensions. The non-renormalizable models are those whose existence requires a finite value of the U.V. cut-off, like QED, the $\phi^{4}$ model in four dimensions, the Standard Model and models in Solid State Physics. It is believed that asymptotically free models are renormalizable only.

The traditional reason to discard non-renormalizable models was their weak predictive power due to the infinitely many renormalization conditions they require. Since we can never be sure what kind of Lagrangian to extrapolate up to high energies we need another point of view. According to the universality scenario the non-renormalizable models can be excluded because they cannot produce anything different from renormalizable theories. The cut-off independent physics of the latter is parametrized by means of renormalized coupling constants. But the subtle point to study below is that this reasoning assumes the presence of a single scaling regime with non-trivial scaling laws in the theory which is a rather unrealistic feature [7].

Thus renormalizability is the requirement of a simple extrapolation to the U.V. regime without encountering "new physics". The evolution of High Energy Physics shows that this is a rather unrealistic assumption, any model with such a feature can be an approximation at best, to study a given interaction and to sacrify the rest for the sake of simplicity. The goals are less ambitious in Statistical Physics and apart of the case of second order phase transitions the renormalizability of models is required seldom, for convenience only, not to carry the regulator through the computation. All models in Solid State Physics are effective ones given with a physically motivated cut-off.

Let us start the discussion of the possible effect of the co-existence of several scaling regimes along the $\mathrm{RG}$ flow with the simplest case, a model with a gap in the excitation spectrum above the ground state, ie with finite correlation length, $\xi<\infty$. Suppose that the RG flow of the model starts in the vicinity of an U.V. fixed point and reaches an IR fixed point region as shown in Fig. 6. Universality, the parametrizability of the physics beyond the U.V. scaling regime by the relevant coupling constants of the U.V. scaling laws, tacitly assumes the absence of any new relevant coupling constants as we move along the renormalized trajectory towards the IR, as shown in Fig. 5. This assumption is in fact correct for massive models whose IR scaling laws are trivial, meaning that that only the Gaussian mass term is relevant. To see why let us consider the RG flow in the IR side of the crossover, when the U.V. cut-off, say the lattice spacing $a>>\xi$ and make a blocking step, $a \rightarrow a^{\prime}$ which generates the change $g_{n}(a) \rightarrow g_{n}\left(a^{\prime}\right)$ in the coupling constants. The change $\Delta g_{n}(a)=g_{n}\left(a^{\prime}\right)-g_{n}(a)$ is due to fluctuation modes whose characteristic length is $a<\ell<a^{\prime}$. Owing to the inequality $\xi<<a<\ell$ these fluctuations are suppressed by $e^{-a / \xi}$ and the flow slows down, $g_{n}(a) \approx g_{n}\left(a^{\prime}\right)$. This implies the absence of run-away trajectories, the absence of relevant non-Gaussian operator. But more realistic models with massless particles or with dynamical or spontaneous symmetry breaking occurring at finite or infinite length scales, respectively, or with condensate in the ground state the IR scaling may generate new relevant operators and universality, as stated in the 


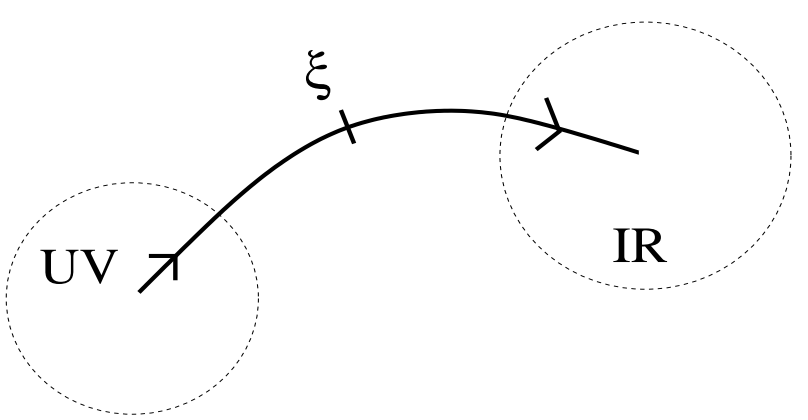

Fig. 6 The RG flow of a model with massive particles. The renormalized trajectory connects the U.V. and IR scaling regimes, indicated by circles which are separated by a crossover at the intrinsic scale of the model, $p \approx 1 / \xi$.

introduction, referring to a single scaling regime is not a useful concept any more.

\subsubsection{Scalar model}

In order to understand better the generic case shown in Fig. 6 we return to its simplest realization, the scalar model in the local potential approximation, Eq. (14), [7]. One does not expect anything unusual here since the model possesses a gap and the IR scaling regime is trivial in either the symmetrical or symmetry broken phase. But we shall see that the model has soft large amplitude fluctuations and the true vacuum is just there where this non-perturbative and the well known perturbative domains join. The second derivative of the local potential is discontinuous there, depends on the direction from which the vacuum is approached. We shall arrive at some understanding of the soft modes in two step, by separating the loop contributions from the tree-level structure. We start with the more traditional loop contributions.

To facilitate the dealing with soft modes we introduce an external constraint which controls the expectation value $\int_{x}\left\langle\phi_{x}\right\rangle_{j}=V \Phi$. The usual realization of this constraint is the introduction of an external source as in Eq. (5). We shall consider the model in the symmetry broken phase where $\int_{x}\left\langle\phi_{x}\right\rangle_{0}=V \Phi_{\text {vac }} \neq 0$ and choose homogeneous external source $j_{x}=J$ which can destabilize the naive symmetry broken vacuum whenever $J \Phi_{\mathrm{vac}}<0$ and can induce $|\Phi|<\Phi_{\mathrm{vac}}$. Note that such a control of the field expectation value by an external source preserves the locality of the action.

The beta-functions, as given by Eq. (23), depend strongly on the propagator $G\left(k^{2}\right)=$ $1 /\left(k^{2}+g_{2}(k)\right)$, represented by the the internal lines in Figs. 3. The qualitative features of the function $G^{-1}\left(k^{2}\right)$ are shown in Fig. 7 for $\Phi=0$. According to the loop expansion

$$
G^{-1}\left(k^{2}\right)=k^{2}+U_{k}^{(2)}(\Phi)= \begin{cases}k^{2}+U_{\Lambda}^{(2)}(\Phi)+\mathcal{O}(\hbar) & U_{k}^{(2)}<<k^{2} \\ k^{2}+U_{0}^{(2)}(\Phi)+\mathcal{O}(\hbar) & k^{2}<<U_{k}^{(2)}\end{cases}
$$

the bending of the lines in the figure is due to radiative corrections, as far as the perturbative region, $G^{-1}>>1$ is concerned. The dashed line corresponds to the massless Coleman-Weinberg case [23]. The flow above or below this separatrix is in the symmetrical or symmetry broken phase, respectively. This qualitative picture is valid so long $|\Phi|<\Phi_{\text {vac }}$. 


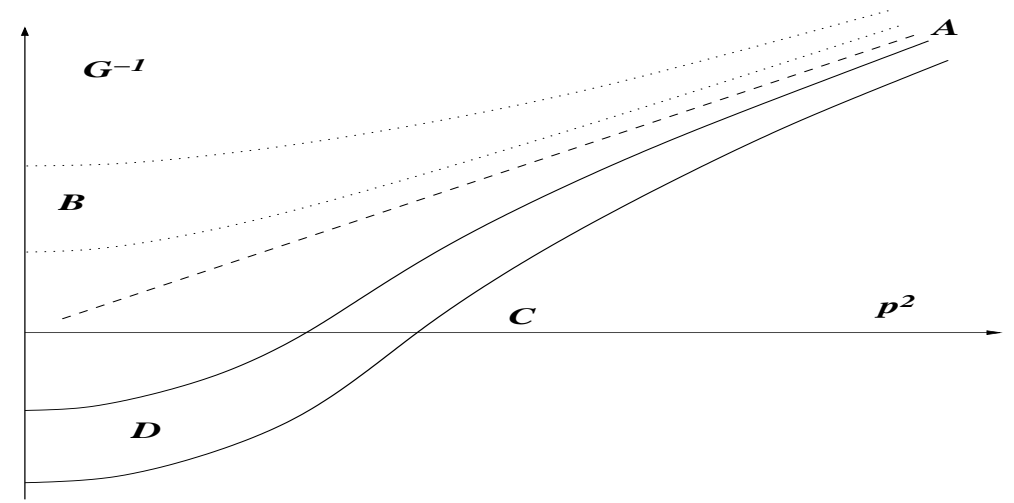

Fig. 7 The inverse propagator $G^{-1}\left(p^{2}\right)$ of the scalar model as the function of the momentum square $p^{2}$ for $\Phi=0$. The dotted and solid lines correspond to the symmetrical and the symmetry broken phase, respectively. The massless case is shown by dashed line. One can distinguish four different scaling regimes, namely A: U.V., B: symmetrical massive, C: precursor of condensation and D: spinodial phase separation regime.

It is important to recall that the argument of the logarithmic function in the WH equation in Eq. (14), $G^{-1}\left(k^{2}\right)$, is the curvature of the action at the cut-off and serves as a measure of the restoring force driving fluctuations back to their trivial equilibrium position. Thus perturbative treatment is reliable and the loop contributions are calculable so long $\tilde{G}^{-1}\left(k^{2}\right)=G^{-1}\left(k^{2}\right) / k^{2}>>\tilde{g}_{4}$.

In order to have a qualitative ides if what is happening let us consider the model where $U_{\Lambda}(\Phi)=m^{2} \phi^{2} / 2+g \phi^{4} / 4$ ! at the cut-off, $k=\Lambda$. By simply ignoring the loop corrections we have $g_{2}(k)=m^{2}, g_{4}(k)=g$ and $g_{n}(k)=0$ for $n>4$ on the treelevel, ie $m^{2}$ and $g$ can be identified with renormalized parameters and $m^{2}<0$. For an isolated system $(j=0)$ where $|\Phi|=\Phi_{\text {vac }}=-6 m^{2} / g$ and the use of the perturbation expansion appears to be justified for $\tilde{G}^{-1}\left(k^{2}\right)=1-m^{2} / k^{2}>g_{4} k^{d-4}$. Spontaneous symmetry breaking, or the appearance of a condensate in the ground state in general, is characterized by instability of fluctuations around the trivial, vanishing saddle point. When an external source is coupled to $\phi_{x}$ to dial $|\Phi|<\Phi_{\text {vac }}$ in the symmetry broken phase then non-perturbative effects set in as $k$ decreases. We can see this easily when the field expectation value is squeezed in the concave part of the potential, $|\Phi|<\Phi_{\text {infl }}=-2 \mathrm{~m}^{2} / \mathrm{g}=$ $\Phi_{\text {vac }} / 3$. In fact, $G^{-1}$ approaches zero and the amplitude of the fluctuations explodes at $k=\sqrt{-m^{2}-g \Phi^{2} / 2}$. Below this non-perturbative regime the plane wave modes become unstable since $G^{-1}\left(k^{2}\right)<0$ and a coherent state is formed, reflected in the appearance of a non-homogeneous saddle point with characteristic scale $1 / \sqrt{-m^{2}-g \Phi^{2} / 2}$

The mechanism responsible of spreading the instability over the whole region $|\Phi|<$ $\Phi_{\text {vac }}$ comes from the tree-level structure and will be discussed later, in section 3.3.

We identify at this point four different scaling regimes:

A: In the U.V. scaling regime $k^{2} \gg g_{2}(k): G_{n} \approx g_{n} / k^{2}$, the mass term is negligible.

B: The explicit scale dependence disappears in the IR regime $k^{2} \ll g_{2}(k)$ of the symmetrical phase, where $G_{n} \approx g_{n} / g_{2}$. This is a trivial scaling, there are no interactive relevant operators.

$\mathrm{C}$ : The onset of the condensation is at $G^{-1}\left(k^{2}\right)=k^{2}+g_{2}(k) \approx 0$ and the higher loop 
effects start to dominate.

D: The spinodal unstable region where the homogeneous ground state becomes unstable against infinitesimal fluctuations, $k^{2}+g_{2}(k)<0$. The leading scaling laws are coming from tree-level, classical physics.

There are two crossover regimes. One is between the scaling regimes $A$ and $B$ at $k^{2} \approx g_{2}(k)$. Another one is between $A$ and $C$ when $|\Phi|<\Phi_{\text {infl. }}$ This will be extended to the region $|\Phi|<\Phi_{\text {vac }}$ by tree-level contributions. We shall look into the crossover of the symmetry broken phase.

The best is to follow the evolution of the local potential the plane $\left(\Phi, k^{2}\right)$, as shown in Fig. 8. The potential $U_{k}(\Phi)$ should be imagined as a surface above the plane $\left(\Phi, k^{2}\right)$. The RG equations for the dimensionless coupling constants, $\dot{\tilde{g}}_{n}=\tilde{\beta}_{n}$ where $\tilde{\beta}_{n}$ is given by Eq. (25) are integrated in the direction of the arrows. The initial condition $\tilde{U}_{\Lambda}(\tilde{\phi})=$ $m^{2} \tilde{\phi}^{2} / 2 k^{2}+g k^{d-4} \tilde{\phi}^{4} / 4$ !, set at $k=\Lambda$, along the horizontal line on the top. As the running cut-off $k$ is decreased and the RG equations are integrated the potential becomes known along horizontal line intercepting the ordinate at $k^{2}$. The spontaneous breaking of the symmetry in the vacuum implies that for sufficiently small $|\Phi|$ the propagator explodes by approaching a singularity at $k=k_{\mathrm{cr}}(\Phi)$ as $k$ decreases. This happens first at $\Phi=0$ when the running horizontal line touches the two curves shown in the Figure. These curves correspond to the generalization of the minimum and the inflection point of the potential for $k^{2}>0$ and are given by the relations $k^{2}+m_{B}^{2}+g \Phi_{\min }^{2} / 6+\mathcal{O}(\hbar)=0$ and $k^{2}+m_{B}^{2}+g \Phi_{\text {infl }}^{2} / 2+\mathcal{O}(\hbar)=0$, respectively. We shall argue in section 3.3 that the singularity lies close to the extension of the minimum,

$$
k_{\mathrm{cr}}\left(\Phi_{\min }(k)\right) \approx k .
$$

The numerical integration of the WH equation requires a truncation of the summation in Eq. (20) for the local potential. By using $n_{\max } \leq 22$ the singular line can be located with reasonable accuracy. Close to the singular line on the plane $\left(\Phi, k^{2}\right)$ the coupling constants increase rapidly and the truncation of the potential is not acceptable. In the U.V. scaling regime (denoted by A in Fig. 7) the propagator is small, $G=\mathcal{O}\left(k^{2}\right)$, and the first term on the right hand side of Eq. (23) is dominant. As we approach $k_{\text {cr }}$ the propagator starts to increase, all term contribute equally in the beta functions and we enter into a new scaling regime ( $\mathrm{C}$ in Fig. 7).

In order to connect the two scaling regimes we shall consider $f_{m, n}(k / \Lambda)=\partial \tilde{\beta}_{m, k}(\phi) / \partial \tilde{g}_{\Lambda, n}$, the dependence of the beta functions at $k$ on the microscopical initial condition, imposed at $k=\Lambda$. The simplest way to estimate $f_{m, n}(x)$ is to start with $f_{m, n}(1) \approx 1$ which can be inferred from the form (23). The scaling law (145) allows us to write

$$
f_{m, n}\left(\frac{k}{\Lambda}\right)=\frac{\partial \tilde{\beta}_{m}(\phi)}{\partial \tilde{g}_{n}(\Lambda)}=\frac{\partial \tilde{\beta}_{m}(\phi)}{\partial \tilde{g}_{n}(k)}\left(\frac{k}{\Lambda}\right)^{-\left[g_{n}\right]}=\mathcal{O}\left(\left(\frac{k}{\Lambda}\right)^{-\left[g_{n}\right]}\right)
$$

up to corrections $\mathcal{O}(\hbar)$, confirming the universality of the U.V. scaling regime. In fact, initial, microscopical value of the the irrelevant, non-renormalizable coupling constants $([g]<0)$ leave vanishing trace on the dynamics as $\Lambda / k \rightarrow \infty$. 


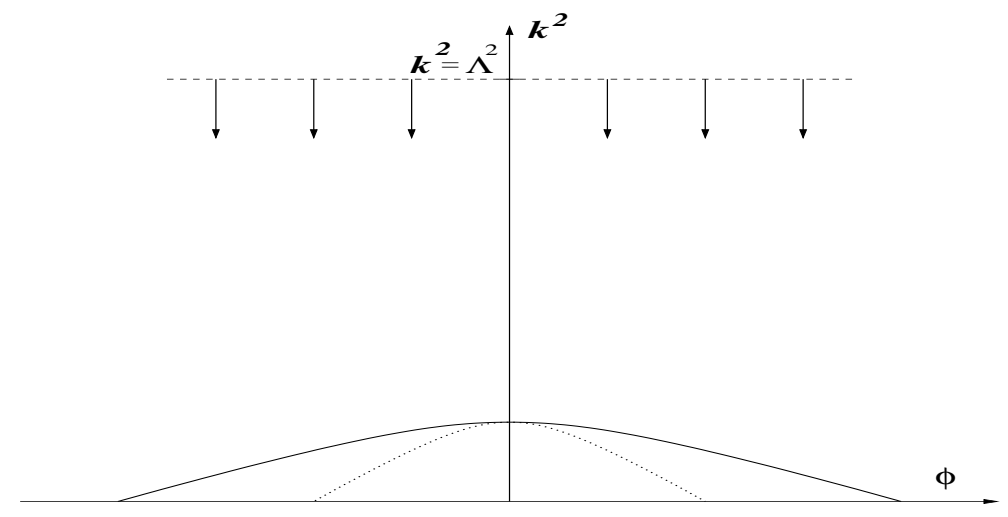

Fig. 8 The $\left(\Phi, k^{2}\right)$ plane where the local potential $U_{k}(\Phi)$ is defined. The potential should be imagined as a surface above the this plan. The initial condition gives $U_{\Lambda}(\Phi)$ on the horizontal dashed line and the RG equation is integrated from this line in the direction of the arrows. The extension of the minimum and the inflection point of the potential from $k^{2}=0$ to $k^{2}>0$ is shown by solid and dotted lines, respectively.

The numerical determination of the function $f_{m, n}(x)$ in $d=4$ supports the prediction (148) in the U.V. scaling regime, namely the impact of a non-renormalizable coupling constants $g_{n}(\Lambda)$ on the beta functions weakens as $(k / \Lambda)^{-\left[g_{n}\right]}$ for $x \approx 1$. But this trend changes for $x<1$ as the critical value of the cut-off, $k_{\mathrm{cr}}$, found to be in agreement with Eq. (147), is approached in what $f$ starts to increase. The potential must not be truncated close to the singularity and the numerical results appear inconclusive. But the increase of $f_{m, n}(x)$ at the crossover actually starts already far enough from $k_{\mathrm{cr}}$ when all couplings are small, $\tilde{g}_{n}<<1$ and the truncation is safe. The lesson of this numerical result is that universality as used around the U.V. scaling regime alone is not valid anymore. The instability at the onset of the condensation at $k \approx k_{\text {cr }}$ introduces divergences which are strong enough to overwrite the U.V. scaling laws and generate new relevant operators. Such an instability of the RG flow enhances the sensitivity of the physics at finite length scales on the microscopical parameters.

The need of generalizing universality in a global manner has been shown for $|\Phi|<\Phi_{\text {vac }}$. It remains to see if such a phenomenon can be observed at the highly singular point $|\Phi|=\Phi_{\text {vac }}$, in the true vacuum.

A similar phenomenon has already been noticed in connection with the BCS ground state. It has been pointed out that kinematical factors turn the four electron operator which is irrelevant according to the power counting into marginal for processes close to the Fermi level [60], [61] [62]. The collinear divergences at the Fermi level drive the instability of the non-condensed vacuum and generate new scaling laws. Another similar mechanism is the origin of the strong long range correlations in the vacuum state of Yang-Mills models. They appear as a consequence of non-renormalizable, U.V. irrelevant Haar-measure term of the path integral [63].

We note finally that the view of the RG flow as a parallel transport, mentioned in section 2.3 is particularly well suited for the studies of crossovers. This is because the sensitivity matrix (78) is a global quantity displaying clearly the sensitivity of the IR physics on the U.V. parameters by construction. 


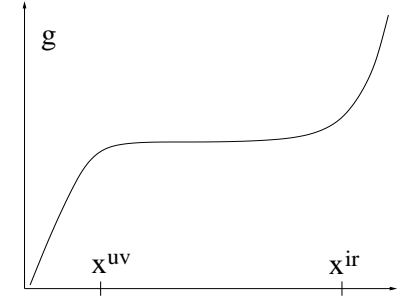

(a)

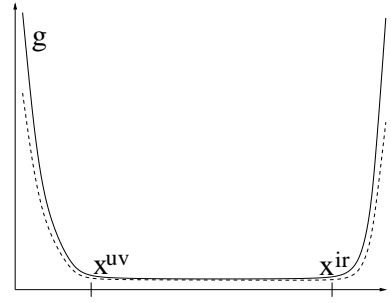

(c)

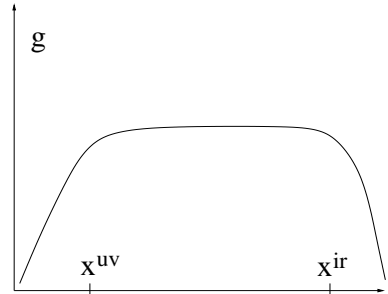

(b)

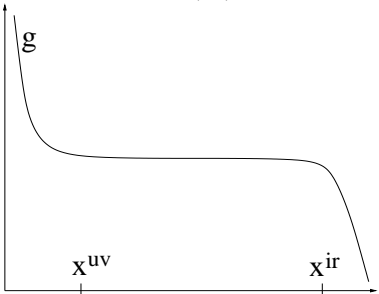

(d)

Fig. 9 The qualitative dependence of the four types of coupling constants on the observational length scale. The U.V. and the IR scaling regimes are $0<x<x^{u v}$ and $x^{i r}<x$, respectively. The dashed line in (c) corresponds to a different initial condition for the RG flow.

\subsubsection{RG microscope}

We now embark on a rather speculative subject, playing with the possibility of matching different scaling laws. Let us start with the generic case, a model with U.V. and IR scaling regimes, separated by a crossover, as sketched in Fig. 6. We have a classification of the very same operator algebra at both scaling regimes. One can write any local operator $A$ as a linear superposition of scaling operators, $A=\sum_{n} c_{A, n} \mathcal{O}_{n}$, the latter being the eigenoperators of the linearized blocking relations of a scaling regime, $\mathcal{O}_{n}(k) \approx(\Lambda / k)_{n}^{\nu} \mathcal{O}(\Lambda)_{n}$. Let us denote by $\nu_{A}$ the largest scaling dimension of scaling operators which occur in the a linear decomposition of $A, \nu_{A}=\max _{n} \nu_{n}$. We shall simplify matters by calling a local $A$ operator relevant, marginal or irrelevant if $\nu_{A}>0, \nu_{A}=0$ or $\nu_{A}<0$, respectively. We ignore marginal case by assuming that radiative corrections always generate nonvanishing scaling dimensions.

One can distinguish between the following four cases:

(a) $r_{\mathrm{U} . V .} r_{\mathrm{IR}}$ : relevant in both regimes,

(b) $r_{\mathrm{U} . \mathrm{V}} i_{\mathrm{IR}}$ : relevant in the U.V., irrelevant in the IR,

(c) $i_{\mathrm{U} . \mathrm{V} .} r_{\mathrm{IR}}$ : irrelevant in the U.V., relevant in the IR,

(d) $i_{\mathrm{U} . \mathrm{V} .} i_{\mathrm{IR}}$ : irrelevant in both regimes.

The qualitative dependence of the corresponding coupling constants is shown in Figs. 9.

Let us consider for example QED containing electrons, muons and ions with metallic density. There is an U.V. scaling regime for energies well above the electron mass. Suppose that the ground state is a BCS type superconductor where the IR scaling laws are driven by long range phonon mediated interactions. The electron mass is an example of the class (a). The muon mass belongs to class (b) owing to the decoupling of muons at low energy. By assuming that radiative corrections turn the four fermion vertex relevant 
it serves as an example of class (c). Finally the six fermion vertex is doubly irrelevant and belongs to class (d).

The interesting class is (c). Suppose that we modify the values of the bare coupling constants at the initial condition and find the dashed line. Due to the universality of the U.V. scaling regime the two renormalized trajectories converge towards each other for $0<x<x^{u v}$ and run very close between the two scaling regimes, $x^{u v}<x<x^{i r}$. But in the IR scaling regime where the coupling constant is relevant the two trajectories start to diverge from each other. The question is whether the difference of the trajectories in the U.V. scaling regime has any impact on the difference found at the IR. It may happen that the IR phenomena is independent of the U.V. regime and the crossover smear completely out the small effects of the U.V. initial condition of the trajectory. But one can imagine that the extremely small differences left at the crossover remain important and lead to an initial condition dependent divergence at the IR. The answer is model dependent and can be given by the detailed analysis of the set of coupled differential equations.

In order to make this point clearer let us imagine a model with two parameters, say a mass $m$ and a coupling constant $g$, both expressed in a dimensionless manner by means of the U.V. cut-off, the lattice spacing $a$. Suppose the following rather simple scaling laws: The beta function of the mass is approximately constant,

$$
\gamma(m, g)=\frac{a}{m} \frac{\partial m}{\partial a} \approx \beta_{m}>0
$$

and of the coupling constant $g$ can be written as

$$
\beta(m, g)=a \frac{\partial g}{\partial a}=\left[\chi\left(\frac{m}{m_{c r}}\right) \nu_{U . V .}+\chi\left(\frac{m_{c r}}{m}\right) \nu_{I . R .}\right] g
$$

where $\chi(z)$ is interpolating smoothly between $\chi(0)=1$ and $\chi(\infty)=0$. The running mass is given by

$$
m(a)=m\left(a_{0}\right)\left(\frac{a}{a_{0}}\right)^{\beta_{m}},
$$

and the length scale of the crossover between the U.V. and the I.R. scaling regimes is at $a_{c r} \approx a_{U . V .}\left(m_{c r} / m_{U . V .}\right)^{1 / \beta_{m}}$. The asymptotical scaling for the coupling constant is

$$
\begin{aligned}
& g\left(a_{U . V .}\right) \approx c_{U . V .} g\left(a_{c r}\right)\left(\frac{a_{U . V}}{a_{c r}}\right)^{-\nu_{U . V .}}, \quad a_{U . V .} \ll a_{c r}, \\
& g\left(a_{I . R .}\right) \approx c_{I . R .} g\left(a_{c r}\right)\left(\frac{a_{I . R .}}{a_{c r}}\right)^{\nu_{I . R .}}, \quad a_{I . R .} \gg a_{c r} .
\end{aligned}
$$

Let us furthermore assume that $\nu_{U . V}<0$ and $\nu_{I . R .}>0$, i.e. this coupling constant belongs to the class $(\mathrm{c})$. The sensitivity of the coupling constant in the asymptotical regions, $g\left(a_{U . V .}\right)$ and $g\left(a_{I . R .}\right)$ on $g\left(a_{c r}\right)$ is

$$
\begin{aligned}
& \frac{\partial g\left(a_{U . V .}\right)}{\partial g\left(a_{c r}\right)} \approx c_{U . V .}\left(\frac{a_{U . V .}}{a_{c r}}\right)^{-\nu_{U . V .}}, \\
& \frac{\partial g\left(a_{I . R .}\right)}{\partial g\left(a_{c r}\right)} \approx c_{I . R .}\left(\frac{a_{I . R .}}{a_{c r}}\right)^{\nu_{I . R .}}
\end{aligned}
$$


therefore

$$
\frac{\partial g\left(a_{I . R .}\right)}{\partial g\left(a_{U . V .}\right)} \approx \frac{c_{I . R .}}{c_{U . V .}} \frac{a_{I . R . R}^{\nu_{I . R}} a_{U . V}^{\nu_{U V} .}}{a_{c r . R .}^{\nu_{I}+\nu_{U . V .}}}
$$

If the IR scaling regime is long enough then the difference between renormalized trajectories with different initial conditions imposed in the U.V. regime can be as large or even larger than at the initial condition. When the U.V. and the I.R. observational scales are related by

$$
a_{U . V .} \approx a_{c r}\left(\frac{a_{c r}}{a_{I . R .}}\right)^{-\nu_{I . R .} / \nu_{U . V .}}
$$

then (154) is $\mathcal{O}\left(a^{0}\right) c_{I . R .} / c_{U . V}$. and despite the 'focusing' of the universality in the U.V. regime the 'divergence' of the trajectories in the I.R. can amplify the extremely weak dependence on the initial condition at finite scales. The increase of $a_{I . R}$. in this case enhances the sensitivity on the initial condition and we can 'see' the initial value of the non-renormalizable coupling constants at smaller distance $a$.

The lesson of the numerical results mentioned above is that the scalar theory in the unstable regime realizes such a RG 'microscope'. The loss of the U.V. based universality as we approach the critical line on the plane $\left(\Phi, k^{2}\right)$ suggests that there is at least one relevant operator at the new scaling laws which is irrelevant in the U.V.. We do not know the eigen-operators of the linearized blocking relations in this region but this new relevant operator must contain the local monomials $\phi_{x}^{n}$ with $n>4$. To make it more difficult, it is not obvious that this is a local operator as opposed to the scaling operators of an U.V. fixed point.

In order to parametrize the physics of the scalar model we have to use this new coupling constant at the U.V. cut-off, as an additional free parameter. This is rather un-practical not only because we do not know the operator in question but mainly due to the smallness of this coupling constants in the U.V. and the crossover region. It seems more reasonable to use a mixed parametrization, consisting of the renormalizable coupling constants at the U.V. cut-off and the new coupling constant taken close to the singularity where it has large enough value, ie $k \approx k_{\mathrm{cr}}$. In the scalar model $k_{\mathrm{cr}} \rightarrow 0$ as the external source is turned off. We shall call the new coupling constants appearing in this manner hidden parameters.

What operator corresponds to the hidden parameter of the scalar model? It seems reasonable to assume that the only observables whose value is determined at the onset of the condensate is just the magnitude of the condensate. Therefore the conjecture is that the strength of the condensate, $\Phi_{v a c}$, is dynamically independent of the renormalizable parameters $g_{2}, g_{4}$ in $d=4$. We have therefore the following possibilities in parametrizing the scalar model with spontaneously broken symmetry:

- We identify a non-renormalizable coupling constant, $g_{n r}$, which influences the value of the condensate and use the bare values of $g_{2}(\Lambda), g_{4}(\Lambda), g_{n r}(\Lambda)$ at the U.V cut-off.

- One uses the renormalizable bare coupling constants from the U.V. end and the strength of the condensate from the I.R. regime, $g_{2}(\Lambda), g_{4}(\Lambda), \Phi_{v a c}$.

- One may use I.R. quantities only, say $g_{2}(0), g_{4}(0), \Phi_{v a c}$. 
Our point is that the scalar model with spontaneously broken symmetry has three free parameters instead of two. This rather surprising conjecture can be neither supported nor excluded by perturbation expansion because one can not connect the U.V. and the I.R. regimes in a reliable manner. The numerical studies of the scalar model carried out so far are inconclusive, as well, because they were constrained to the quartic potential and the possible importance of higher order vertices in forming the condensate was not considered.

The modification of the scaling laws of the scalar model, necessary to generate a hidden parameter comes from the onset of a condensation. As discussed below in Section 3.3 there are non-trivial tree-level scaling laws in a condensate which overwrite the loopgenerated beta functions and may provide the dynamical origin of the hidden parameter. In a similar manner one can speculate about the role of the Cooper-pair condensate in the BCS vacuum. If there turns out to be a hidden parameter as in the one-component scalar model then the non-renormalizable effective coupling constants of the Standard Model would influence the supercurrent density in ordinary metals! Another possible example is the Higgs sector of the Standard Model, where the hidden parameter, if exists, would be an additional free parameter of the model.

One may go further and inquire if dynamical symmetry breaking can modify the scaling law in a similar manner. The bound state formation, responsible to the generation of large anomalous dimensions in strong coupling QED [64], in strong extended technicolor scenario [65, 66], in the Nambu-Jona-Lasinio model [67] or in the top-quark condensate mechanism $[68,66]$ may be a source of hidden parameters, as well.

Hidden parameters represent an unexpected 'coupling' between phenomena with very different scales and question of our traditional strategy to understand a complex system by analyzing its constituents first. The axial [70] or scale [71] anomalies represent a well know problem of this sort, except that the regulator independence of the anomaly suggests that for each classical IR fixed point there is a single 'anomalous' one without continuous fine tuning.

Universality is expressed in the context of High Energy Physics by the decoupling theorem [69]. Let us start with a renormalizable model containing a heavy and a light particle and consider the effective theory for the light particle obtained by eliminating the heavy one. There are two possible classification schemes for the effective vertices for the light particle, generated by the elimination process. One is according to the U.V. scaling laws in the effective theory, i.e. there are renormalizable and non-renormalizable vertices. Another scheme is based on the strength of the effective interactions, i.e. there are vertices which are stay constant or diverge when the ratio of the light and the heavy particle mass tends to zero and there are vertices whose coefficients tend to zero in the same limit. The decoupling theorem asserts that these two classification schemes are equivalent, i.e. the non-renormalizable effective coupling constants are vanishing when the heavy mass diverges. The existence of the hidden parameter is, at the final count is a violation of this theorem. 


\subsubsection{The Theory of Everything}

The appearance of a hidden coupling constant renders our goal of understanding the hierarchy of interactions in Nature extremely complicated. In fact, the RG flow of the Theory of Everything must be imagined in the space of all coupling constants in Physics, including the parameters of the Grand Unified Models down to different models in Solid State Physics or hydrodynamics in the classical regime, as indicated in Fig. 10. Different fixed points are approached by the trajectory when the scale dependence is dominated by a single interaction. But all fixed point except the U.V. one is avoided due to the non-renormalizable, irrelevant vertices [69] generated by the dynamics of the next fixed point in the U.V. direction.

Such a wandering in the space of coupling constants echoes the age old disagreement between High Energy and Solid State Physics. It is usually taken for granted in the High Energy Physics community that the sufficiently precise determination of the microscopical parameters of the Theory of Everything would 'fix' the physics at lower energies. The obvious difficulties to extract the high energy parameters from experiments which render each new experiment expensive, long and large scale operation indicate that this might not be a practical direction to follow. In other words, the relevant coupling constants have positive Lyapunov exponent and render the trajectory extremely sensitive on the initial conditions. Therefore the characterization of the RG flow of The Theory of Everything by the initial condition, though being possible mathematically, is not practical due to the finite resolution of the measurements. The other side of the coin, the physical parameters of a fixed point are clearly the relevant coupling constants of the given scaling regime. They are the object of Solid State Physics, as far as the scaling regimes QED, CM and IR are concerned in Fig. 10. One looses sight of the fundamental laws but gains predictive power by restricting oneself to local studies of the RG flow.

\subsection{Instability induced renormalization}

In the traditional applications of the $\mathrm{RG}$ method which are based on the perturbation expansion the RG trajectory is sought in the vicinity of the Gaussian fixed point. One can describe in this manner crossovers which connect scaling regimes which share the same small parameter. We can enlarge the classes of accessible crossovers by relying on the saddle point expansion, the only systematical non-perturbative approximation scheme. The generic way to induce new crossover in this scheme is by passing a condensation at a finite length scale [72].

One may object that there is nothing surprising or new in finding that the physics is fundamentally changed by a condensation mechanism. What additional knowledge can then be gained by looking into condensation by the method of the RG? The point is that the classification of operators around a fixed point which was achieved in the framework of the perturbation expansion changes essentially by the condensation, ie the appearance of a non-trivial saddle point. To see how this happens we return to the strategy followed at Eq. (2). Let us consider an observable $A$ in a model with a single coupling constant $g$ for 


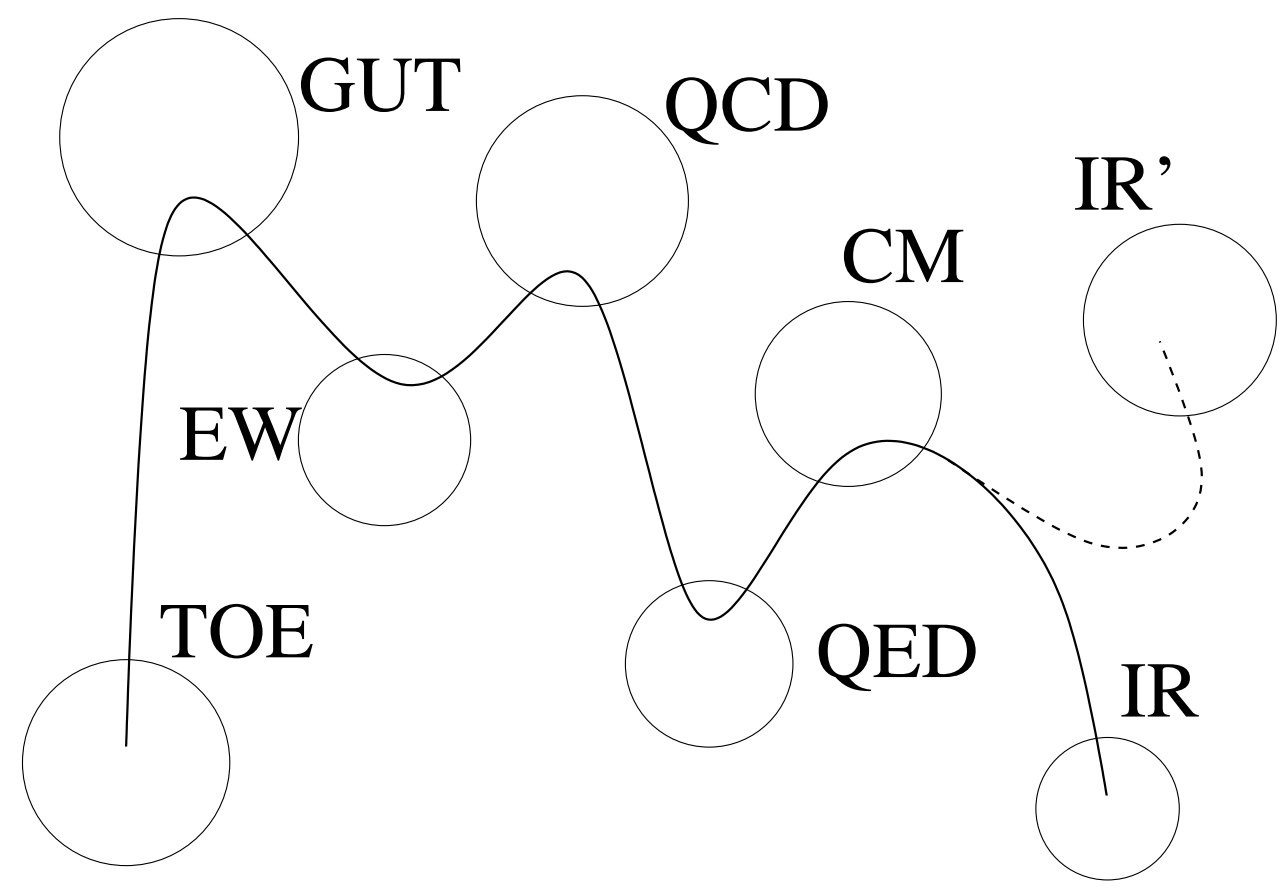

Fig. 10 The renormalization group flow of the Theory of Everything. The branching drawn at an IR scale is a phase transition driven by the environment, such as particle or heat reservoirs.

simplicity, computed in the saddle point expansion, $\langle A\rangle=F_{0}(g, \Lambda)+\hbar F_{1}(g, \Lambda)+\mathcal{O}\left(\hbar^{2}\right)$, where $F_{\ell}(g, \Lambda)$ denotes the $\ell$-loop contribution. We can obtain the beta function by taking the derivative of this equation with respect to cut-off $\Lambda$,

$$
\beta=k \partial_{k} g=-\frac{k \partial_{k}\left(F_{0}+\hbar F_{1}\right)}{\partial_{g}\left(F_{0}+\hbar F_{1}\right)}+\mathcal{O}\left(\hbar^{2}\right)=-\frac{k \partial_{k} F_{0}}{\partial_{g} F_{0}}\left[1+\hbar\left(\frac{\partial_{k} F_{1}}{\partial_{k} F_{0}}-\frac{\partial_{g} F_{1}}{\partial_{g} F_{0}}\right)\right]+\mathcal{O}\left(\hbar^{2}\right)
$$

The loop corrections $F_{\ell}(g, \Lambda), \ell \geq 1$ are polynomials of the coupling constants $g$. But the leading order, tree-level piece usually has stronger dependence on $g$ and may become singular as $g \rightarrow 0$. The tree-level contribution may induce qualitatively new scaling laws with new set of relevant operators. Due to the singularity at $g=0$ the condensation actually realizes the dangerous irrelevant variable scenario [73].

At the onset of an instability, region $\mathrm{C}$ in Fig. 7 certain modes experience strong nonlinearity and develop large amplitude fluctuations. But as we enter in the unstable regime one may hope to recover quasiparticles with weak residual interaction after having settled the dynamics of the unstable modes. At least this is what happens within the framework of the semiclassical, or loop expansion where the fluctuations remain small after the proper condensate is found. There are models where the vacuum state is just at the edge of the instability, like the scalar model discussed above in the symmetry broken phase.

The tree-level contributions to the blocking transformation, if exist, are more important then the loop corrections. This is the reason that we shall consider the tree-level, $\mathcal{O}\left(\hbar^{0}\right)$ RG flow. There is no evolution in this order so long the system is stable and the saddle point is trivial. But as soon as we arrive at the unstable region the RG flow becomes non-trivial. 


\subsubsection{Unstable effective potential}

Before turning to the actual tree-level blocking let us review briefly the kind of instabilities one expects. As we enter into the unstable regime $k^{2}+g_{2}(k)<0$ the trivial saddle point, $\phi_{k}=0$ becomes unstable in the blocking (7). To understand this instability better we introduce the constrained functional integral,

$$
Z_{\Phi}=\int D[\phi] e^{-S_{B}[\phi]} \delta\left(\frac{1}{V} \int_{x} \phi_{x}-\Phi\right)
$$

corresponding to a conserved order parameter. By following the RG trajectory until the IR end point one eliminates all but the homogeneous mode by keeping the partition function unchanged,

$$
Z_{\Phi}=e^{-S_{k=0}[\Phi]}=e^{-V U_{k=0}(\Phi)} .
$$

To find another local representation for this constrained partition function we consider the generator functional density $w[j]=W[j] / V$ defined by means of Eq. (5) and compute its Legendre transform, $V_{\text {eff }}(\Phi)$ in the mean field approximation. For this end we set $j_{x}=J$ and write

$$
-V_{\mathrm{eff}}(\Phi)=w[J]-J \Phi=\min _{J, \phi}\left[-U_{k=0}(\phi)+J(\phi-\Phi)\right] .
$$

The minimization with respect $\phi$ and $J$ yields $J=d w(J) / d J$ and and $\phi=\Phi$, respectively. Finally we have $V_{\text {eff }}(\Phi)=U_{k=0}(\Phi)$, the result announced after Eq. (16) so long the mean field approximation is reliable, namely in the thermodynamical limit and in the absence of large amplitude fluctuations. It is further known that $V_{e f f}(\Phi)$ is convex.

The instabilities of the kind mentioned above are well known in the case of first order phase transitions. But similar instabilities may appear at higher order phase transitions, as well. In fact, the magnetization of the Ising model as the function of the external magnetic field shows discontinuous behavior below the critical temperature. Therefore the free energy constrained into a sector with a given magnetization displays such kind of instabilities.

Suppose that the $U_{k=0}(\Phi)$ determined perturbatively has degenerate minima and a concave part. Then it is advantageous to introduce two curves on the $(\Phi, k)$ plane of Fig. $8, \Phi_{\text {infl }}(k)$ and $\Phi_{\min }(k)$ defined by $k^{2} \Phi_{\min }+U_{k}^{(1)}\left(\Phi_{\min }\right)=0$, and $k^{2}+U_{k}^{(2)}\left(\Phi_{\text {infl }}\right)=0$. The stable region in the mean-field approximation is $|\Phi|>\Phi_{\min }(0)=\Phi_{\text {vac }}$. For $\Phi_{\text {inf }}(0)<$ $|\Phi|<\Phi_{\min }(0)$ there are two minima in $\phi$ for the last equation in (159). One of them is metastable, ie is unstable against sufficiently large amplitude modes. The spinodal phase separation, the instability against infinitesimally small amplitude fluctuations occurs when some eigenvalues of the second functional derivative of the action becomes negative. This is region D in Fig. $7,|\Phi|<\Phi_{\text {infl }}(0)$. It is unreachable by the mean-field treatment because no local minimum is found in $\phi$ which would satisfy $|\phi|<\Phi_{\text {infl }}(0)$. To understand better the nature of these unstable regions one has to go beyond the mean-field approximation and to follow the dynamics of the growing inhomogeneous instabilities. We shall use the tree-level WH equation to deal with such large amplitude, inhomogeneous fluctuations. 


\subsubsection{Tree-level WH equation}

The construction of the condensate within the unstable region implies the minimization of the action with respect to a large number of modes. The RG strategy offers an approximation for such a rather involved problem, it deals with the modes one-by-one during the minimization [75], [74].

The negative curvature of the potential makes the saddle point in (9) non-trivial,

$$
S_{k-\Delta k}[\phi]=\min _{\tilde{\phi}^{\prime}} S_{k}\left[\phi+\tilde{\phi}^{\prime}\right]=S_{k}\left[\phi+\tilde{\phi}_{c l}[\phi]\right] \neq S_{k}[\phi] .
$$

Since the saddle point depends in general on the background field, $\tilde{\phi}_{c l}=\tilde{\phi}_{c l}[\phi]$, the action is modified during the blocking and we find a non-trivial RG flow [74]. The local potential approximation to the tree-level blocking is

$$
U_{k-\Delta k}(\Phi)=\min _{\tilde{\phi}_{c l, x}} \int_{x}\left[\frac{1}{2}\left(\partial_{\mu} \tilde{\phi}_{c l, x}\right)^{2}+U_{k}\left(\Phi+\tilde{\phi}_{c l, x},\right)\right] .
$$

One encounters here a conceptual problem. The $k$-dependence of the tree-level RG flow might well be singular since there is no obvious reason that the saddle points which are usually rather singular functions of the parameters evolve smoothly. This problem has already been noticed as a possible 'first order phase transition' in the blocking which induces discontinuous RG flow [76], [77], [78]. It was later shown by rigorous methods that the RG flow is either continuous or the blocked action is non-local [79]. The resolution of this apparent paradox is that the saddle point actually develops in a continuous manner as we shall shown below. As the RG flow approaches the onset of the condensate, $k \rightarrow k_{c r}$ then the new scaling laws generate such an action that the saddle point turns out to be continuous in $k$.

\subsubsection{Plane wave saddle points}

The saddle points, the minima in (160) satisfy the highly non-linear Euler-Lagrange equations whose solutions are difficult to find. The use of sharp cut-off slightly simplifies the problem since it reduces the functional space in which the minimum is sought to $\tilde{\phi} \in \mathcal{F}_{k} \backslash \mathcal{F}_{k-\Delta k}$. We shall retain the the plane wave saddle points only,

$$
\tilde{\phi}_{c l, p}=\frac{\rho_{k}}{2}\left[e^{i \theta_{k}} \delta_{p, k e_{k}}+e^{-i \theta_{k}} \delta_{p,-k e_{k}}\right], \quad \tilde{\phi}_{c l, x}=\rho_{k} \cos \left(k e_{k} \cdot x+\theta_{k}\right) .
$$

The parameter $\theta_{k}$ and the unit vector $e_{k}$ correspond to zero modes, they control the breakdown of translational and rotational symmetries.

This is a key to what happens at the unstable line: Despite the discreteness of the internal symmetry $\phi \rightarrow-\phi$ there are soft modes because the inhomogeneous saddle points break the continuous external symmetries. The condensation of particles with non-vanishing momenta automatically generates Goldstone modes. This phenomenon is well known for solids where the saddle point is a crystal of solitons which breaks external symmetries and there is no integration over the zero mode to restore the symmetrical 
ground state. Our point is that the soft modes make their appearance even if the symmetry of the ground state is restored by the integration over the zero modes.

The amplitude $\rho_{k}$ is determined by minimization,

$$
U_{k-\Delta k}(\Phi)=\min _{\rho_{k}}\left(\frac{1}{4} k^{2} \rho_{k}^{2}+\frac{1}{\pi} \int_{0}^{\pi} d y U_{k}\left(\Phi+\rho_{k} \cos y\right)\right),
$$

in the local potential approximation. The numerical implementation of this iterative procedure to find the $\mathrm{RG}$ flow with an initial condition imposed on the potential at $k=\Lambda$ gave the following results [74]:

(1) The recursive blocking relation, Eq. (163) is not a finite difference equation. Despite of this the flow converges as $\Delta k \rightarrow 0$.

(2) The amplitude of the saddle point satisfies the equation $\Phi+\rho(k)=\Phi_{\min }(k)$.

(3) The potential obtained by the tree-level blocking is $U_{k}(\Phi)=-\frac{1}{2} k^{2} \Phi^{2}$.

(4) The tree-level results above hold independently of the choice of the potential at the cut-off.

The key is point 3, point 1 follows immediately from it. The lesson of this result is that the action is degenerate for the modes at the cut-off, the kinetic and potential energies cancel. The 'best' effective theory for a given plane wave mode is the one whose cut-off $k$ is slightly above the wave vector of the mode. This result suggests the degeneracy of the action within the whole unstable region. Conversely, if we can show that the action is degenerate at the cut-off within the unstable regime we established this potential.

We prove by induction in the number of steps $k \rightarrow k-\Delta k$ that the variation of the action density within the unstable regime is $\mathcal{O}(\Delta k)$. This result protects the consistency of the saddle point expansion for $d>1$ since $\Delta k \geq 2 \pi / L$ where $L$ is the size of the system and therefore the variation of the action is $\mathcal{O}\left(L^{\bar{d}-1}\right)$. Recall that $k_{c r}$ denotes the cut-off where the kinetic snd the potential energies cancel.

First step: Let us denote by $k^{\prime}$ the value of the cut-off at the first occurrence of nontrivial saddle point in the numerical implementation of Eq. (163). It obviously satisfies the inequality $k_{\mathrm{cr}}-\Delta k<k^{\prime}<k_{\mathrm{cr}}$. The trivial saddle point $\tilde{\phi}_{\mathrm{cs}}=0$ becomes unstable for blockings with $|\Phi|<\Phi_{\text {infl }}\left(k^{\prime}\right)$ in which case $\left|\Phi+\tilde{\phi}_{\mathrm{cs}, x}\right|<\Phi_{\text {infl }}\left(k^{\prime}\right)$, ie $\left|\tilde{\phi}_{\mathrm{cs}, x}\right|=\mathcal{O}(\sqrt{\Delta k})$. The term $\mathcal{O}(\tilde{\phi})$ is canceled in the action on a homogeneous background field so the $\mathcal{O}\left(\tilde{\phi}_{\text {cs }}^{2}\right)$ contribution gives $\mathcal{O}(\Delta k)$ variation.

Induction: Suppose that the variation of the action is $\mathcal{O}(\Delta k)$ in the unstable region and we lower the cut-off, $k \rightarrow k-\Delta k$. At the new cut-off the balance between the kinetic and the potential energy is lost by an amount of $\mathcal{O}(\Delta k)$ since the potential energy is still the given by $k$ but the kinetic energy corresponds to the lowered cut-off, $k-\Delta k$. Thus the negative curvature potential energy wins and the action bends downward in the unstable region as the function of the amplitude of the plane wave. By assuming that the amplitude is stabilized at an $\mathcal{O}\left(\Delta k^{0}\right)$ value (will be checked later) the variation of the action density $\mathcal{O}(\Delta k)$ in the unstable region.

Point 2 can be understood in the following manner: During the minimization of the action $\rho_{k}$ slides down on the $\mathcal{O}(\Delta k)$ slope until the potential starts to increase again. We can find where this happens by equating the slope of the original, bare potential with that 
stated in point 3 . One would expect that the deepest point is reached at $\rho_{k}=\Phi_{\text {infl }}(k)$. But the $\mathcal{O}\left(\phi^{4}\right)$ term in $U_{k}(\phi)$ is not yet strong enough to make the potential increase strong enough here. The two slopes agree just at $\rho_{k}=\Phi_{\min }(k)$.

Result 4 which follows from 3 , as well, can be called super universality since it reflects scaling laws where all coupling constants are irrelevant. Note that the $k$-dependence is continuous through the whole RG trajectory.

Point 3 is a generalized Maxwell construction. It reduces to the traditional Maxwell construction for $k=0$, to the degeneracy of $U_{k=0}(\Phi)$ for $|\Phi|<\Phi_{\min }(0)=\Phi_{\mathrm{vac}}$. The naive Maxwell cut, applied for the concave part of the potential would give the degeneracy for $|\Phi|<\Phi_{\text {infl }}$ only. The problem with this argument is that it produces an effective potential which is convex everywhere except at $|\Phi|=\Phi_{\text {infl }}(0)$. The second derivative of the potential is ill defined and the first derivative is discontinuous at this point. By placing the system into a finite box the singularity is rounded off and the second derivative becomes finite, but turns out to be negative in a vicinity of $|\Phi|=\Phi_{\text {inf }}(0)$. Convexity regained only if the cut is extended between the minima, $|\Phi|<\Phi_{\min }(0)$.

The generalized Maxwell construction in the mixed phase of a first order phase transition can be understood by the dynamics of the domain walls. The flatness of certain thermodynamical potentials in the mixed phase reflects the presence of zero-modes, the location of domain walls. Such a rather simple kinematical mechanism which is independent of microscopic details is the source of the 'super universality', point 4 above. The role of the domain walls is played by saddle points in our computation, the cosine function in (162) realizes infinitely many equally spaced, parallel domain walls. The integration over the zero modes $\theta_{k}$ and $e_{k}$ according to the rules of the saddle point expansion restores translational and rotational symmetries of the ground state and reproduces the mixed phase.

One might object our independent treatment of the plane wave saddle points. The RG equation (163) handles the plane waves in the consecutive momentum space shells independently which seems as a vast oversimplification. But one should recall at this point that according to the general strategy of the RG method the dynamics of the modes eliminated during the blocking is retained by the modification of the effective coupling constants. This is not always possible since there are more modes than coupling constants, the problem mentioned at the end of section 2.1.1. More precisely, the general framework of the blocking what is in principle always applicable is turned into a powerful scheme when an approximation is made. One truncates the effective action and assumes that the solution of this over-determined problem exists. This is the main, and so far unproven, assumption of the RG method. Accepting this point we can determine the blocked action by means of a homogeneous background field and use it for non-homogeneous field configurations at the next blocking step as written in Eq. (163).

\subsubsection{Correlation functions}

One can compute the tree-level contributions to the correlation functions in a combination of the mean-field and the saddle point approximation [80]. Let us split the complete 
functional integral into the sum of contributions which come from the unstable and the stable regions. The fluctuations from the stable regions are governed by a nontrivial action and they are taken into account as loop corrections. The fluctuations in the unstable region experience a flat action and their contributions must be taken into account on the tree-level. The complication of integrating up these contributions is the determination of the region where the action is flat. Our approximation consists of estimating this region for each plane wave independently, ie extending the integration over the amplitude $r_{p}$ of the degenerate plane wave

$$
\tilde{\phi}_{c l}(x)=r_{p} \cos \left(p e_{p} x+\theta_{p}\right)
$$

over the interval $-\rho_{p}<r<\rho_{p}$, where $\rho_{p}$ is given by (162). Let us denote the integration over the resulting domain by $D_{\Phi}[r]$ and write the correlation function in momentum space in this single-mode determined flat region as

$$
\begin{aligned}
G_{\text {tree }}^{\Phi}(p, q)= & \frac{1}{4}\left[\int D[\theta] D[e] D_{\Phi}[r]\right]^{-1} \int D[\theta] D[e] D_{\Phi}[r] r_{p} r_{q} \\
& \times\left(e^{i \theta_{p}} \delta_{p, k e_{p}}+e^{-i \theta_{p}} \delta_{p,-k e_{p}}\right)\left(e^{i \theta_{q}} \delta_{q, k e_{q}}+e^{-i \theta_{q}} \delta_{q,-k e_{q}}\right) .
\end{aligned}
$$

Due to the flatness of the action these integrals are purely kinematical and can easily be carried out. All integration whose variable does not show up in the integrand drops out. The integration over the shift of the plane waves, the phase angle $\theta$, restores the translation invariance,

$$
\begin{aligned}
G_{\text {tree }}^{\Phi}(p, q) & =\delta_{p+q, 0}\left[\int d e \int d r_{p}\right]^{-1} \int d e \int d r_{p} r_{p}^{2} \\
& =\delta_{p+q, 0} \frac{2(2 \pi)^{d} d}{3 \Omega_{d}} \frac{\left(\Phi_{\min }(p)-\Phi\right)^{2}}{\Phi_{\min }^{-1}(\Phi)}
\end{aligned}
$$

where $\Phi_{\min }^{-1}(\Phi)$ is defined as $\Phi_{\min }\left(\Phi_{\min }^{-1}(\Phi)\right)=\Phi$. The Fourier transform of this propagator describes a diffraction type oscillation with characteristic length scale $\xi=k_{c r}^{-1}$, the characteristic feature of domains in a homogeneous state.

\subsubsection{Condensation as crossover}

The scaling laws of the scalar model change already approaching the condensation in the stable region $C$ of Fig. 7. Inside the instable region $D$ the action with the potential given by point 3 of section 3.3.3 becomes invariant under blocking, ie the whole region $D$ is an IR fixed point.

The vacuum of the scalar model is a single, homogeneous coherent state consisting of zero momentum particles when $|\Phi|>\Phi_{\text {vac }}$. When $|\Phi|<\Phi_{\text {vac }}$ we encounter the singularities as seen above. This singularity may not be real, it might be smoothened out by higher order vertices and a saddle points appearing for $k<k_{\mathrm{cr}}$. But the vacuum is the superposition of inhomogeneous coherent states with characteristic scales $\ell>1 / k_{\mathrm{cr}}$. Each of them breaks space-time symmetries but their sum remains symmetrical. 
One may wonder if any of the discussion applies to the true vacuum $|\Phi|=\Phi_{\text {vac. }}$. For a weakly coupled system the fluctuations are small and one may hope that the fluctuations around $\Phi_{\text {vac }}$ are stable and un-influenced by what is happening with the unstable modes $\phi_{x}<\Phi_{\mathrm{vac}}$. But the answer to this question is rather uncertain and is open because the true vacuum is just at the border of the instabilities and the typical fluctuations around the true vacuum $\left(\phi_{x}-\Phi_{\mathrm{vac}}\right) \approx g^{2}(0) / g_{4}(0)$ penetrate into the unstable region.

The issue of the modification of the scale dependence by a condensate [64] is rather general and points far beyond the simple scalar model.

The vacuum of asymptotically free models supports strong correlations at long distance. In the case of 4 dimensional Yang-Mills models the naive, perturbative vacuum is destabilized by the one-loop level effective action which predicts that the vacuum is made up by a coherent state of gluons [82]. But this state cannot be the true vacuum. The problem is not only that the field generated by the condensate is strong and spoils the saddle point expansion but it turns out that there is an unstable mode. The true vacuum is supposed to be found at even lower energy densities where the long range fluctuations restore the the external and color symmetry broken by a homogeneous condensate of the charged spin one gluons [83] and the vacuum is thought to contain domains of homogeneous field in a stochastic manner [84]. This scenario is close to the view of the mixed phase of the scalar model developed here with the difference that the instability comes from the loop or the tree-level renormalized action in the Yang-Mills or scalar model, respectively. Furthermore the instability in the Yang-Mills model can be avoided by extreme environment only, by immersing the system into strong external field or bringing into contact with heat or particle reservoir.

Similar instability occurs in vacua containing condensate of bound states. The BCS vacuum is made homogeneous in a non-trivial manner when represented in terms of the electrons making up the Cooper pairs. The spontaneous breakdown of the chiral invariance in QCD manifests itself in the condensate of quark-anti quark pairs which is homogeneous after integrating out the instanton zero modes only [85]. The crossover from the U.V. scaling regime to the instability takes place at $k \approx 1 / \ell$ where $\ell$ is the size of the bound states. Higher order derivative terms appearing as effective vertices may generate similar crossover, as well [72], [86].

Finally it is worthwhile mentioning the tunneling phenomena, the dynamical extension of the instabilities considered above. The interesting feature of the dynamical realization of the tunneling by means of time-dependent, tree-level instabilities is that they take place in conservative systems but the long time distribution agrees with the equilibrium predictions coming from the canonical ensemble [81].

\subsection{Sine-Gordon model}

It is not unusual that one arrives at periodic or variables in the construction of effective theories, of non-linear sigma models or non-Abelian gauge theories. Such variables represent a double challenge. One problem which can be considered as local in the in- 
ternal space is that the perturbation expansion around a minima of the potential should keep infinitely many vertices in order to preserve the periodicity. Another, more difficult, global problem is a conflict between the two requirements for the effective potential for periodic variables, namely periodicity and convexity. One can fulfill both requirements in a trivial manner only, by constant effective potential. If true, this conclusion has far reaching consequence for the phenomenological description of such systems. As a case study we shall consider the simplest model with periodic variable, the two dimensional sine-Gordon model [87], [88].

\subsubsection{Zoology of the sine-Gordon model}

The sine-Gordon model

$$
L_{\mathrm{SG}}=\frac{1}{2}\left(\partial_{\mu} \phi\right)^{2}+u \cos \beta \phi
$$

has been shown to be equivalent with the X-Y model [89], [90], [87], the Thirring model [91], [92] and a Coulomb gas [93]. The methods of Refs. [90], [91] and [92], approximate duality transformation, bosonization and semiclassical approximation are valid in certain regions of the coupling constant space. The maps used in Refs. [87] and [93] are exact. The mapping [87] $\psi_{x}=e^{\beta \phi_{x}}$ transforms the model (167) into the compactified sine-Gordon model

$$
L_{\mathrm{CSG}}=\frac{1}{2 \beta^{2}} \partial_{\mu} \psi^{*} \partial_{\mu} \psi+\frac{u}{2}\left(\psi+\psi^{*}\right) .
$$

The models (167) and (168) are equivalent in any order of the perturbation expansion in continuous space-time where the configurations are assumed to be regular.

The X-Y model which is characterized by the action

$$
S_{\mathrm{XY}}=-\frac{1}{T}\left[\sum_{<x, x^{\prime}>} \cos \left(\theta_{x}-\theta_{x^{\prime}}\right)+h \sum_{x} \cos \left(\theta_{x}\right)\right]-\ln z \sum_{x} m_{x}^{2},
$$

where $T=\beta^{2}, h / T=u, z$ is the vortex fugacity and $m_{x}$ denotes the vortex density. The $\mathrm{RG}$ equation of the X-Y model obtained in the dilute vortex gas limit [89], [90]

$$
a \frac{d T}{d a}=4 \pi^{3} z^{2}-\pi T^{2} h^{2}, \quad a \frac{d h}{d a}=\left(2-\frac{T}{4 \pi}\right) h, a \frac{d z}{d z}=\left(2-\frac{\pi}{T}\right) z .
$$

The X-Y model appears as a generalization of the compactified sine-Gordon model since the lattice regularization transforms the Lagrangian (168) into (169) with $z=1$. What is surprising here is that the vortex fugacity is a relevant operator in the high temperature phase of the X-Y model but it is entirely missed by the lattice regularization! Does that mean that the sine-Gordon model Lagrangians (167) and (168) are incomplete?

The vortex term could be missed by the regulator because the plane $z=0$ is RG invariant. Therefore it is consistent to exclude vortices from the path integral in the sine-Gordon model given in continuous space-time. When we recast the model in lattice regularization without ever thinking about vortices our lattice action neither suppresses, nor enhances the singular configurations and sets $z=1$. But once the vortices are not suppressed, $z \neq 0$ the renormalized trajectories moves away from from the plane of fixed 
$z$. There is still no problem in the low temperature phase because all what happens is that an irrelevant coupling constant became fixed. This is how regulators work. But the problem is more serious in the high temperature phase. The Lagrangians (167) or (168) can not give account of this phase because they lack a relevant, renormalizable coupling constant. The perturbative continuum theory without vortices is consistent but once vortices are available kinematically their density evolves in a non-trivial manner.

There is no problem with the lattice regulated X-Y model because the vortex fugacity gets renormalized due to the cut-off dependence of the vortex action. In other words, we can move along the RG flow by adjusting $T$ and $h$ only, the renormalization of $z$ is carried out 'automatically' in the partition function. The renormalization of $z$ is a problem in the continuous formalism only where the vortices are introduced formally as point-like charges.

This is an unexpected mechanism which brings the singular nature of the field configurations into play during renormalization and may plague any quantum field theory. The knowledge of the classical action in continuous space-time leaves open the possibility that we have of adjust the fugacity of certain localized singularities or topological defects not considered in the continuum.

It is interesting to speculate about similar phenomenon in QCD. Lattice QCD is constructed with $\ln z=0$ where $z$ is the fugacity of some topological defects, like instantons, monopoles, merons etc. These topological defects and singularities are supposed to play an important role in the confining vacuum, as vortices do in the high temperature phase of the X-Y model. The renormalization of the fugacities of these objects should first be studied in lattice QCD in order to construct a continuum description of the vacuum.

The sine-Gordon model possesses a topological current, $j_{\mu, x}=\beta \epsilon_{\mu \nu} \partial_{\nu} \phi_{x} / 2 \pi$ which is obviously conserved when the path integral is saturated by field configurations with analytic space-time dependence. Its flux, the vorticity, gives the soliton number. The world lines of the sine-Gordon solitons end at the X-Y model vortices, cf Fig. 11. The distance between the bound vortex-anti vortex pairs shrinks with the lattice spacing in the low temperature continuum limit. Any measurement with finite resolution loose sight of the instability of solitons in the renormalized theory. The average distance between vortices stays finite, cut-off independent in the continuum limit of the high temperature phase and the soliton decay can be observed. One expects the breakdown or at least important modification of the bosonization transformation in this phase. In fact, the nonconservation of the topological current requires fermion number non-conserving terms in the fermionic representation, a fundamental violation of the rules inferred from the weak coupling expansion.

\subsubsection{Effective potential}

In order to understand better the dynamics of the long wavelength modes we compute the effective potential in the sine-Gordon model. We follow the WH method truncated to the local potential approximation which picks up the tree level evolution, too. It is obvious that period length of the potential $2 \pi / \beta$ remains $R G$ invariant in this approximation. 


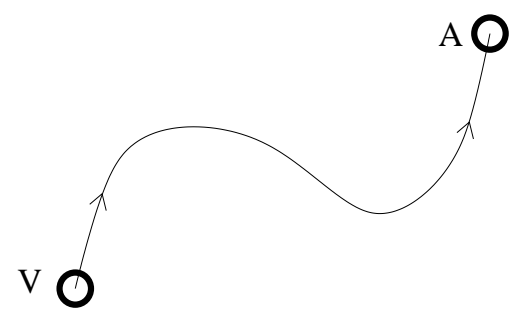

Fig. 11 The world line of a soliton starts at a vortex (V) and ends at an anti-vortex (A) in the two dimensional space-time.

The tree ands the loop level RG equations are

$$
U_{k-\Delta k}(\phi)=\min _{\rho}\left[\frac{k^{2}}{4} \rho^{2}+\int_{-1}^{1} d u U_{k}(\phi+\rho \cos (\pi u))\right]
$$

and

$$
k U_{k-\Delta k}(\phi)=k U_{k}(\phi)+\Delta k \frac{k^{2}}{4 \pi} \ln \left[k^{2}+U_{k}^{(2)}(\phi)\right]
$$

in the plane $z=0$.

We use the Fourier expanded form for the potential

$$
U_{k}(\phi)=\sum_{n=0}^{\infty} u_{n}(k) \cos (n \beta \phi)
$$

and compute the leading order contribution to the WH equation when expanded in the potential,

$$
k \partial_{k} \tilde{u}_{n}=\left(\frac{\beta^{2} n^{2}}{4 \pi}-2\right) \tilde{u}_{n},
$$

in terms of the the dimensionless coupling constants $\tilde{u}_{n}=u_{n} / k^{2}$. This agrees with the second equation in (170). The solution of (174) is

$$
\tilde{u}_{n}(k)=\tilde{u}_{n}(\Lambda)\left(\frac{k}{\Lambda}\right)^{\frac{\beta^{2} n^{2}}{4 \pi}-2} .
$$

The coupling constants $u_{n}$ are irrelevant in the disordered phase $T=\beta^{2}>8 \pi$ and the effective potential obtained for $k=0$ is flat. The coupling constants $n<8 \pi / T$ are relevant in the ordered phase and the effective potential is non-trivial. At this pointe one suspects that Maxwell construction interferes with the evolution because a non-trivial periodic functions necessarily has concave regions. To settle this question one has to rely on the numerical solution of the evolution equation (171). We followed the loopsgenerated evolution (172) from the initial condition set at $k=\Lambda$ until the stability is lost at $k=k_{\text {cr }}$ for background field values which lie at local maxima of the periodic potential. For $k<k_{\text {cr }}$ equation (171) was used. The result is that the coupling constants approach zero as $k \rightarrow 0$ due to the plane wave saddle points. The effective potential is trivial in both phases. 


\subsubsection{Breakdown of the fundamental group symmetry}

The local potential $U_{k}(\phi)$ flattens without developing singularities in the high temperature disordered phase. The transformation

$$
\phi_{x} \rightarrow \phi_{x}+\frac{2 \pi}{\beta}
$$

is a discrete symmetry of the action and is preserved in the vacuum since the potential barrier between the minima is vanishing for long range modes. Since we are at the lower critical dimension, $d=2$, the large amplitude long range modes realize the 'tunneling' between the minima of the periodic potential. On the contrary to this situation, the potential develops discontinuous second derivatives in the ordered, low temperature phase and the instability driven flattening of the potential $U_{k}(\phi)$ reflects the survival of barriers between the minima of the potential. All this looks like a spontaneous symmetry breaking, therefore our conclusion is that the transformation (176) is not a symmetry of the vacuum in the ordered, low temperature phase.

The usual circumstance under which such a phenomenon arises is the multiple connectedness of the internal space. In the present context the non-linear $U(1) \sigma$-model parametrization, Eq. (168), is based on the internal space $U(1)$, with the fundamental group $Z$ generated by the transformation (176). The dynamical breakdown of the fundamental group symmetry is a genuine quantum effect. In fact, the path integral formally extends over all homotopy classes, this is the symmetrical phase. When the dynamics develops sufficiently high barriers between the homotopy classes the path integral becomes restricted to a single homotopy class.

It is important to recall that the time evolution described by the Schrodinger equation can be derived from the path integral by performing infinitesimal variations on the end point of the trajectory. Therefore the consistency of the dynamics can be maintained when the path integration is restricted into any functional subspace which is closed under continuous deformation of the trajectories. The loss of the interference between homotopy classes is the characteristic feature of the symmetry broken phase.

Such symmetry breaking is the key to understanding the way quarks become deconfined at high temperature or the droplet phase is formed for quantum liquids [94]. The configuration space for global gauge transformations is $S U(3) / Z_{3}$ for gluons, with the fundamental group $Z_{3}$. The quark propagator vanishes in the symmetrical phase due to the destructive interference between the three homotopy classes. In the $Z_{3}$ symmetry broken deconfined phase there is no interference and quarks can be observed. Quantum liquids in the first quantized formalism display similar symmetry breaking pattern. The coordinate space for $N$ particles is $R^{3 N} / S_{N}$ where $S_{N}$ consists of permutations of the particles. The absence of the overlap among states belonging to different droplets suppresses the (anti)symmetrization of the states and reduces the exchange symmetry of the ground state.

How can we recognize the dynamical breakdown of the fundamental group symmetry? The simplest strategy, to look for the minima of the effective potential fails because 
Maxwell construction hides any structure in a periodic potential. The answer to this question lies in the topological structure of excitations and the order parameter will be a topological susceptibility.

This is rather natural since the phase transition is the restriction of the path integral into a single homotopy class, the imposition of a topological constraint. Let us assume that the analysis sketched above for the plane $z=0$ remains qualitatively valid for $z \neq 0$, ie the effective potential is always flat, due to the loop-generated evolution or the Maxwell construction in the disordered or ordered phase, respectively. The topological invariant characterizing the homotopy classes is the soliton number. In the weak coupling expansion (ordered phase) the path integral is constrained into a single homotopy class hence the soliton number is conserved, its susceptibility is vanishing in the continuum limit. The stability of the soliton, based on the continuity of the time evolution is lost in the disorder phase because the short distance, large amplitude fluctuations of the typical field configuration extends the path integral all soliton number sector. Therefore the susceptibility of the soliton number is non-vanishing in this phase.

\subsubsection{Lower critical dimension}

It is worthwhile noting the manner the Mermin-Wagner-Coleman theorem [95] appears in the framework of the local potential approximation. By setting $g_{2}=0$ for $d=2$ the linear part of $\mathcal{P}_{n}$ in Eqs. (23) provides a finite evolution but the higher order terms make the renormalized trajectory to diverge as $k \rightarrow 0$ unless the coupling constants approach zero. We can construct interactive, massless models in two dimensions so long the running coupling constants approach zero sufficiently fast in the IR regime as in the case of the sine-Gordon model, presented above. The retaining of the terms $\mathcal{O}\left(\partial^{2}\right)$ in the gradient expansion provides another mechanism to suppress the IR divergences by generating singular wave function renormalization constant, $Z_{k}$ as $k \rightarrow 0$ [96].

In both cases the way to avoid the non-interactive system predicted by the MerminWagner-Coleman theorem is to use the RG improved perturbation expansion. In the ordinary perturbation series we have the contributions like

$$
g^{n} \int_{p_{1}, \cdots, p_{k}} f_{p_{1}, \cdots, p_{k}}
$$

and the integral is IR divergent in $d=2$. One has running coupling constants, $g \rightarrow g_{P}$, in the RG improved perturbation expansion where the analogous contribution is

$$
\int_{p_{1}, \cdots, p_{k}} g_{P\left(p_{1}, \cdots, p_{k}\right)}^{n} f_{p_{1}, \cdots, p_{k}}
$$

When the IR fixed point is Gaussian, $\lim _{P \rightarrow 0} g_{P}=0$, then the theory remains interactive and the running coupling constants suppress the IR divergences.

\subsection{Gauge models}

The blocking step of the RG scheme, being scale dependent, violates local symmetries which mix modes with different length scales. 
The most natural way to deal with this breakdown of gauge invariance is to cancel the gauge non-invariant terms by fine tuning non-invariant counterterms [97]. But this is possible in perturbation expansion only. Another, better suited strategy to the RG method is to argue that the way Ward identities are violated by blocking shows that the BRST invariance is broken by the running cut-off only and the BRST symmetry is recovered at the IR fixed point when the cut-off is removed [20], [98]. This were certainly a good procedure if it could be implemented without further truncation. In a weakly coupled model such as QED one could construct approximations with controllable errors. In asymptotically free models like QCD non-perturbative long range correlations make any scheme which imposes gauge invariance in an approximate manner unreliable [63]. The demonstrate this point consider the static force law between two test charges in a Yang-Mills theory in a scheme where the gauge invariance, Gauss' law is implemented approximately. The gauge non-invariant components of the vacuum state appear charged, by definition. Thus we have an uncontrollable color charge distribution around the test charges. When the distance between the test charges are large enough then it will be energetically favorable for the uncontrollable charges to break the flux tube. We loose the string tension in a manner similar how it happens in the true QCD vacuum due to the virtual quark-anti quark polarizations. In more formal words, an arbitrarily weak gauge-dependent perturbation can change the long range features of the vacuum as in the ferromagnetic phase of the Ising model.

The most natural way to guarantee gauge invariance is to achieve independence on the choice of gauge by using gauge invariant quantities only. The proposal of Ref. [99] goes along this line but the use of loop variables renders the computation rather involved. We discuss now a version of the RG scheme in the internal space which at least for Abelian models produces the effective action without any gauge fixing. The electron mass combined with chiral transformation has already been used in generating evolution equation in Ref. [100]. We shall follow a simpler and more general by avoiding gauge dependence in an explicit manner for the photon Green functions [101]. The gauge invariant electron composite operators are not difficult to include [41].

\subsubsection{Evolution equation}

Let us consider the generator functional

$$
e^{\frac{1}{\hbar} W[j, \bar{j}, J]}=\int D[\bar{\psi}] D[\psi] D[A] e^{\frac{1}{\hbar} \int_{x}\left[-\frac{1}{4 e^{2}} F^{\mu \nu} F_{\mu \nu}-\frac{\alpha}{2}\left(\partial_{\mu} A^{\mu}\right)^{2}+\bar{\psi}(i \not D-m) \psi+\bar{j} \cdot \psi+\bar{\psi} \cdot j+J^{\mu} \cdot A_{\mu}\right]}
$$

where $D_{\mu}=\partial_{\mu}+i A_{\mu}$ and the dimensional regularization is used to render $W$ finite. We control the quantum fluctuations by modifying the action, $S \rightarrow S+S_{\lambda}$ with

$$
S_{\lambda}=\frac{\lambda}{4 e^{2}} \int_{x} A_{\mu, x} \square T^{\mu \nu} A_{\nu}(x),
$$

where $T_{\mu \nu}=g_{\mu \nu}-\partial_{\mu} \partial_{\nu} / \square$. The infinitesimal change of the control parameter $\lambda \rightarrow \lambda+\Delta \lambda$ modifies the free photon propagator

$$
D^{\mu \nu}(x-y) \rightarrow D^{\mu \nu}(x-y)+\Delta \lambda \int d z D^{\mu \rho}(x-z) \square_{z} T_{\rho \kappa} D^{\kappa \nu}(z-y)+\mathcal{O}\left((\Delta \lambda)^{2}\right),
$$


in a manner reminiscent of the Callan-Symanzik scheme.

The evolution equation (108) with the present suppression takes the form

$$
\partial_{\lambda} \Gamma_{\lambda}[A]=\frac{\hbar}{2 e^{2}} \operatorname{tr}\left[\square T^{\mu \nu}\left(\frac{\delta^{2} \tilde{\Gamma}[A]}{\delta A_{\mu} \delta A_{\nu}}\right)^{-1}\right]
$$

in terms of the photon effective action $\tilde{\Gamma}_{\lambda}[A]=\Gamma_{\lambda}[A]+S_{\lambda}[A]$. We project the evolution equation on the functional space given by the ansatz

$$
\tilde{\Gamma}[A]=\frac{1}{2} \int_{x} A_{\mu, x} D^{-1}(i \partial) T^{\mu \nu} A_{\nu, x}+\mathcal{C}[A]
$$

where

$$
D^{-1 \mu \nu}=-\frac{1+\lambda}{e^{2}} \square T^{\mu \nu}-\alpha \square L^{\mu \nu},
$$

$L^{\mu \nu}=\delta^{\mu \nu}-T^{\mu \nu}$ and $\mathcal{C}[A]$ is a gauge invariant functional.

The control parameter $\lambda$ 'turns on' the fluctuations of the photon field. Therefore the electrons loop contributions to the effective action must already be present at the initial condition which is chosen to be $\mathcal{C}[A]=-i \operatorname{tr} \ln (i \not D-m)$ at $\lambda=\lambda_{0}$.

The second functional derivative matrix is written as

$$
\frac{\delta^{2} \tilde{\Gamma}[A]}{\delta A_{\mu} \delta A_{\nu}}=D^{-1 \mu \nu}+\frac{\delta^{2} \mathcal{C}[A]}{\delta A_{\mu} \delta A_{\nu}}
$$

and the inversion is carried out by expanding in the non-diagonal pieces to write the evolution equation as

$$
\partial_{\lambda} \mathcal{C}[A]=\frac{\hbar}{2 e^{2}} \operatorname{tr}\left[\square T^{\mu \nu} D \sum_{n=0}^{\infty}(-1)^{n}\left(\frac{\delta^{2} \mathcal{C}[A]}{\delta A_{\mu} \delta A_{\nu}} D\right)^{n}\right]
$$

\subsubsection{Gauge invariance}

We show now that the limit $\alpha \rightarrow 0$ can be taken in the evolution equation without hitting any singularity. The gauge fixing parameter $\alpha$ enters through the photon propagator $D$ in the Neuman-expansion of the right hand side of Eq. (186). The $\alpha$-dependent longitudinal contributions of the first and the last $D$ factor are suppressed by the gauge invariance of the suppression term, represented by the transverse projection $T^{\mu \nu}$ in (186). The longitudinal photon contributions of the internal propagators are suppressed by the gauge invariance of the effective action,

$$
\partial_{\mu} \frac{\delta \mathcal{C}[A]}{\delta A_{\mu}}=0 .
$$

According to this equation $\delta^{2} \mathcal{C}[A] / \delta A_{\mu} \delta A_{\nu}$ does not mix the longitudinal and the transverse modes, ie non-longitudinal contributions appear in the evolution equation.

We choose a gauge in the argument above what was relaxed at the end of the computation. But the steps followed remain well defined even if we start with $\alpha=0$, without 
any gauge fixing. Our argument about the decoupling of the longitudinal and transverse contributions to the evolution equation still applies but it is not clear if the longitudinal part was well defined. This subtle issue is settled by Feynman's $\epsilon$ parameter, devised to lift infinitesimally the degeneracies of the action. It is easy to see that it gives a weak variation of the action along gauge orbits by breaking gauge invariance. As long as our truncation of the evolution equation, the functional $\mathcal{C}[A]$ is explicitly gauge invariant $\epsilon$ plays the role of an infinitesimally external magnetic field in the Ising model, ie helps the breakdown of the gauge symmetry only if it is really broken in the true vacuum.

\section{What has been achieved}

The traditional implementation of the RG idea has proven to be essential in Statistical and High Energy Physics, starting with the understanding of critical phenomena [102], finite size scaling [103], $\epsilon$-expansion [104], dynamical processes with different time scales [105], continuing with partial resummation of the perturbation expansion [4], parametrizing the scale dependence at high energies [106] and ending with the construction of effective theories in Particle Physics [107, 108, 109]. This is an inexcusable short and incomplete list, its role is to demonstrate variability and the importance of the method only. Our main concern here was the functional form of the RG method and its generalizations. From this point of view one may distinguish conceptual and more technical achievements as the power of the functional formalism is more exploited.

We should consider the RG method as 'meta-theory', or in more practical terms as a language, as Feynman graphs are used in particle physics. But this language is not bound by small parameters and can provide us a general, non-perturbative approximation method beyond the semiclassical expansion and numerical simulations.

The path integration was first viewed as a powerful book-keeping device for perturbation expansion and the truly non-perturbative application came later, after having gained some experience with the formalism. This is similar to the development of the functional $\mathrm{RG}$ method where the RG equation refers to the generator function of the effective coupling constants and provides us with a simple procedure to keep track of the Feynman graphs. The steps in functional calculus are more cumbersome because they deal with generator function(al)s, with infinitely many coupling constants. The ultimate goal is to go beyond this level and to use this formalism in a genuinely non-perturbative manner.

The semiclassical expansion is our first step in this direction. It will be important to check how the RG continues to be applicable where the saddle point expansion ceases to be reliable.

Another field the RG scheme might be compared with is lattice regulated field theory. Both are general purpose tools to deal with non-perturbative systems. The bottle-neck of the numerical simulations on the lattice is the need to send the U.V. and the IR cut-offs sufficiently far from each others and the restriction to Euclidean space-time. The RG strategy is set up in continuous, Minkowski space time and there is no particular problem with keeping the U.V. and IR cut-offs far from each other. But the drawback of the RG 
strategy is that it is rather lengthy to extend the space of (effective) action functionals used in the computation. Since the limitations of the two methods are quite different they might be used in a complementary manner.

The functional formalism is promising because of the possibility of following the mixing of a much larger number of operators as in the traditional strategy. This feature gives the hope of extending the applicability of the method from a single scaling regime to the whole range of scales covered by the theory. Such an extension may provide us valuable information about the competition of interactions in realistic theories.

\section{Acknowledgments}

I thank Jean Alexandre and Kornel Sailer for several useful discussions during our collaboration.

\section{References}

[1] N.N. Bogoliubov, D.V. Shirkov, Introduction to the Theory of Quantized Fields, 3rd ed., John Wiley and Sons, New York, 1973.

[2] K.G. Wilson, J. Kogut, Phys. Rep. C 12, 77 (1974); K.G. Wilson, Rev. Mod. Phys. 47, 773 (1975); Rev. Mod. Phys. 55, 583 (1983)

[3] P.W. Anderson, G.J. Yuval, J. Phys. C 4, 607 (1971).

[4] M. Gellman, F. Low, Phys. Rev. 95, 1300 (1954).

[5] N. Goldenfeld, O. Martin, V. Oono, and F. Liu, Phys. Rev. Lett. 64, 1361 (1990); T. Kunihiro, Progr. of Theor. Phys. 95, 503 (1995).

[6] C. G. Callan, Phys. Rev. D 2, 1541 (1970); K. Symanzik, Comm. Math. Phys. 18, $227(1970)$.

[7] J. Alexandre, V. Branchina, J. Polonyi, Phys. Rev. D 58, 16002 (1988).

[8] F.J.Wegner, A.Houghton, Phys. Rev. A 8, 401 (1973).

[9] J. Polchinski, Nucl. Phys. B 231, 269 (1984).

[10] T. Morris, Int. J. Mod. Phys. A9 (1994) 2411.

[11] J. Collins, Renormalization, Cambridge University Press, 1984.

[12] J. Polonyi, K. Sailer, Phys. Rev. D 63, 105006 (2001).

[13] J. Hughes and J. Liu, Nucl. Phys. B 307, 183 (1988); J Hughes, Nucl. Phys. B 312, 125 (1989); G.M. Shore, Nucl. Phys. B 362, 85 (1991); G. Keller and C. Kopper, Comm. Math. Phys. 148, 445 (1992); Comm. Math. Phys. 153, 245 (1993); C. Becchi, On the Construction of Renormalized Gauge Theories Using Renormalization Group Techniques, hep-th/9607188.

[14] B.P. Dolan, Int. J. of Mod. Phys. 10, 2439 (1995); Int. J. of Mod. Phys. 10, 2703 (1995);Int. J. of Mod. Phys. 12, 2413 (1995); D.C. Brody, A. Ritz, Nucl. Phys. B 522, 588 (1998); B.P. Dolan, A. Lewis, Phys. Lett. B 460, 302 (1999). 
[15] G. Altarelli, G. Parisi, Nucl. Phys. B 126, 278 (1977); A. H. Mueller, Phys. Rep. 73, 237 (1981).

[16] C. Wetterich, Phys. Lett. B 301, 90 (1993).

[17] T. Morris, Phys. Lett. B 329, 241 (1944).

[18] D. Litim, Optimized renormalization group flow, hep-th/0103195.

[19] M. Reuter and C. Wetterich, Nucl. Phys. B 391, 147 (1993); N. Tetradis and D.F. Litim, Nucl. Phys. B 464, 492 (1996).

[20] U. Ellwanger, Phys. Lett. B 335, 364 (1994).

[21] J. Adams, J. Berges, S. Bornholdt, F. Freire, N. Tetradis, C. Wetterich, Mod. Phys. Lett. A 10, 2367 (1995).

[22] J. Alexandre, J. Polonyi, Ann. Phys. 288, 37 (2001).

[23] S. Coleman, E. Weinberg, Phys. Rev. D 7, 1888 (1973).

[24] A. Parola, L. Reatto, Phys. Rev. Lett. 53, 2417 (1984); Phys. Rev. A 31, 3309 (1985); V. I. Tikar, Phys. Lett. A 104, 135 (1984); J. Comellas, A. Travesset, Nucl. Phys. B 498, 539 (1997).

[25] K. I. Aoki et al, Progr. of Theor. Phys. 95, 409 (1996).

[26] S. B. Liao, M. Strickland, Nucl. Phys. B 497, 611 (1997).

[27] A. Margaritis, G. Ódor, A. Patkos, Z. Phys. C 39, 109 (1998)

[28] P. E. Haagensen, Y. Kubyshin, J. I. Latorre, E. Moreno, Phys. Lett. B 323, 330 (1994)

[29] T. Morris, Phys. Lett. B 334, 355 (1994).

[30] N. Tetradis, C. Wetterich, Nucl. Phys. B 422, 541 (1994).

[31] M. Alford, Phys. Lett. B 336, 237 (1994).

[32] S. Liao, J. Polonyi, M. Strickland, Nucl. Phys. B 567, 493 (2000).

[33] D. F. Litim, Phys. Lett. B 486, 92 (2000); Int. J. of Mod. Phys. 16, 2081 (2001).

[34] S. Seide, C. Wetterich, Nucl. Phys. B 562, 524 (1999).

[35] J. Berges, C. Wetterich, Nucl. Phys. B 487, 675 (1997).

[36] M. Maggiore, Z. Phys. C 41, 687 (1989); T. E. Clarke, B. Haeri, S. T. Love, Nucl. Phys. B 402, 628 (1993); M. D'Attanasio, T. Morris, Phys. Lett. B 409, 363 (1997);

J. Berges, D. U. Jungnickel, C. Wetterich, Phys. Rev. D 59, 034010 (1999);

[37] D. Zanchi, H. J. Schultz, Phys. Rev. B 54, 9509 (1996); Phys. Rev. B 61, 13609 (2000); C. J. Halboth, W. Metzner, Phys. Rev. B 61, 4364 (2000); C. Honerkamp, M. Salmhofer, N. Furukawa, T. M. Rrice, Phys. Rev. B 63][45114, 2001 (;) M. Salmhofer. C. Honerkamp, Progr. of Theor. Phys. 105, 1 (2001); S. Correia, J. Polonyi, J. Richert, Functional Callan-Symanzik Equation for the Coulomb Gas, cond-mat/0108048.

[38] J. Polonyi, Current-density functional for disordered systems, cond-mat/0203090.

[39] A. Bonanno, M. Reuter, Phys. Rev. D 62, 043008 (2000). 
[40] H. Gies, C. Wetterich, Renormalization group flow of bound states, hep-th/0107221.

[41] J. Polonyi, K. Sailer, Effective actions for the density functional theory, condmat/0108179.

[42] C. Bagnuls, C. Bervillier, hep-th/0002034; Berges, N. Tetradis, C. Wetterich, hepth/0005122.

[43] Proceedings of Workshop on the Exact Renormalization Group, Faro, Portugal, 1998, ed. Krasnitz et al. (World Scientific,Singapore, 1999); 2nd Conference on Exact RG, Rome 2000, ed. S. Arnone, at al. Int. J. of Mod. Phys. 16, 1809 (2001).

[44] C. Harvey-Fros, PhD Thesis, university of Southampton, hep-th/0108018; M. D. Turner, PhD Thesis, University of Southampton, hep-th/0108031.

[45] F. Wegner, J. Phys. C 7, 2098 (1974).

[46] J. I. Latorre, T. Morris, Int. J. of Mod. Phys. 16, 2071 (2001).

[47] L. Schulmann, Techniques and Applications of Path Integrations, John Wiley and Sons, New York, 1981.

[48] D. McLaughlin, L. Schulman, J. Math. Phys. 12, 2520 (1971).

[49] J. Polonyi, Ann. Phys. 252, 300 (1996).

[50] R. D. Ball, P. E. Haagensen, J. I. Latorre, E. Moreno, Phys. Lett. B 347, 80 (1995);

[51] J. Comellas, Phys. Lett. B 509, 662 (1998).

[52] T. Morris, Nucl. Phys. B 495, 477 (1997); Phys. Rev. Lett. 77, 1658 (1996).

[53] A. Hasenfratz, P. Hasenfratz, Nucl. Phys. B 270, 687 (1986).

[54] K. Halpern. K. Huang, Phys. Rev. D 35, 3252 (1996); Phys. Rev. Lett. 77, 1659 (1996); V. Periwal, Mod. Phys. Lett. A 11, 2915 (1996); K. Halpern, Phys. Rev. D 57, 6337 (1998).

[55] G. Felder, Comm. Math. Phys. 111, 101 (1987); A. E. Filippov, S. A. Breus, Phys. Lett. A 158, 300 (1981); S. A. Breus, A. E. Filippov, Physica A 192, 486 (1993); T. Morris, Phys. Rev. Lett. 1658, 1996 (.)

[56] T. Morris, Phys. Lett. B 329, 241 (1994).

[57] C. Bagnulus, C. Bervillier, Phys. Rev. B 41, 402 (1990).

[58] T. Morris, Phys. Lett. B 345, 139 (1995);

[59] R. Jackiw, S. Templeton, Phys. Rev. D 23, 2291 (1981).

[60] J. Polchinski, in Proceedings of the 1992 TASI Elementary Particle Physics, Eds. J. Polchinski and J. Harvey, World Sci., 1992.

[61] S. Weinberg, Nucl. Phys. B 413, 567 (1994).

[62] R. Shankar, Rev. Mod. Phys. 66, 129 (1994).

[63] J. Polonyi, Nucl. Phys. (Proc. Suppl.) B20 (1991) 32; Confinement and Renormalization, hep-ph/9511243, in the Proceedings of the International School of Physics, Enrico Fermi, Course CXXX, on Selected Topics in Nonperturbative QCD, Soc. It. di Fisica; K. Johnson, L. Lellouch, J. Polonyi, Nucl. Phys. B 367, 675 (1991). 
[64] V. A. Miransky, Nuovo Cimento A 90, 149 (1985); P.I. Fomin, V. P. Gusynin, V. A. Miransky, Yu. A. Sitenko, Riv. Nuovo Cimento 6, 1 (1983); C. N. Leung, S. T. Love, W. A. Bardeen, Nucl. Phys. B 273, 649 (1986); W. A. Bardeen, S. T. Love, C. N. Leung, Nucl. Phys. B 323, 493 (1989).

[65] T. Appelquist, T. Takeuchi, M. B. Einhorn, L. C. R. Wijewardhana, Phys. Lett. B 220, 223 (1989); K. Matumoto, Progr. of Theor. Phys. 81, 277 (1989); T. Takeuchi, Phys. Rev. D 40, 2697 (1989).

[66] V. A. Miransky, M. Tanabashi, K. Yamawaki, Phys. Lett. B 221, 177 (1989); Mod. Phys. Lett. A 4, 1043 (1989).

[67] V. A. Miransky, Int. J. of Mod. Phys. 6, 1641 (1991).

[68] Y. Nambu, Proceedings of the 1989 Workshop on Dynamical Symmetry Breaking, eds. T. Muta, K. Yamawaki (Nagoya University, 1990); W. J. marciano, Phys. Rev. Lett. 62, 2793 (1989);Phys. Rev. D 41, 1647 (1990); W. A. Bardeen, C. T. Hill, M. Lindner, Phys. Rev. D 41, 1647 (1990).

[69] V. A. Alessandrini, H. de Vega, F. Schaposnik, Phys. Rev. B 10, 3906 (1974); Phys. Rev. B 12, 5034 (1975); T. Appelquist, J. Carazzone, Phys. Rev. D 11, 2865 (1975); T. Appelquist, J. Carazzone, H. Kluberg-Stern, M. Roth, Phys. Rev. Lett. 36, 768 (1976), Phys. Rev. Lett. 36, 1161 (1976); T. Appelquist, J. Carazzone, Nucl. Phys. B 120, 77 (1977).

[70] J. S. Bell, R. Jackiw, Nuovo Cimento A 60, 47 (1969); S. Adler, Phys. Rev. 177, 2426 (1969).

[71] S. Adler, J. Collins, A. Duncan, Phys. Rev. D 15, 1712 (1977); J. Collins, A. Duncan, S. Joeglekar, Phys. Rev. D 16, 438 (1977); N. K. Nielsen, Nucl. Phys. B 120, 212 (1977).

[72] V. Branchina, J. Polonyi, Nucl. Phys. B 433, 99 (1995).

[73] M. E. Fischer, in Proceedings of the Conference Renormalization Group in Critical Phenomena and Quantum Field Theory, edt. J. D. Gunton, M. S. Green, Temple University, 1973.

[74] J. Alexandre, V. Branchina, J. Polonyi, Phys. Lett. B 445, 351 (1999).

[75] A. Ringwald, C. Wetterich, Nucl. Phys. B 334, 506 (1990); N. Tetradis, C. Wetterich, Nucl. Phys. B 383, 197 (1992).

[76] R. B. Griffith, P. A. Pierce, Phys. Rev. Lett. 41, 917 (1978); J. Stat. Physics. 20, 499 (1979); O. Hudák, Phys. Lett. A 73, 273 (1979); C. Rebbi, R. H. Swendsen, Phys. Rev. B 21, 4094 (1980); R. B. Griffith, Physica A 106, 59 (1981); A. Stella, Physica A 108, 211 (1981).

[77] A. Hasenfratz, P. Hasenfratz, Nucl. Phys. B 295, 1 (1988)

[78] H. W. Blöte, R. H. Swendsen, Phys. Rev. Lett. 43, 799 (1979); C. Lang, Nucl. Phys. B 280, 255 (1987); K. Decker, A. Hasenfratz, P. Hasenfratz, Nucl. Phys. B 295, 21 (1988); A. Gonzales-Arroyo, M. Okawa, Y. Shimizu, Phys. Rev. Lett. 60, 487 (1988); F. Igloi, C. Vanderzande, Physica A 135, 347 (1986).

[79] R. B. Israel, in Random Fields (Esztergom, 1979), J. Fritz, J. L. Lebowitz, D. Szász eds. (North-Holland, Amsterdam, 1981); A. C. D. van Enter, R. Fernández, A. Sokal, J. Stat. Physics. 72, 879 (1993). 
[80] J. Alexandre, J. Polonyi, Tree-level renormalization, hep-th/9906017.

[81] Sz. Borsanyi, A. Patkos, J. Polonyi, Zs. Szep, Phys. Rev. D 62, 085013 (2000).

[82] S. Matinyan, G. Savvidy,Nucl. Phys. B 134, 539 (1978); G. Savvidy, Phys. Lett. B 71, 133 (1977); M. Duff, R. Ramón-Medrano, Phys. Rev. D 12, 3357 (1976); H. Pagels, E. Tomboulis, Nucl. Phys. B 143, 485 (1978).

[83] N. K. Nielsen, P. Olesen, Nucl. Phys. B 144, 376 (1978); Phys. Lett. B 79, 304 (1978); J. Amjorn, N. K. Nielsen, P. Olesen, Nucl. Phys. B 152, 75 (1979); H. B. Nielsen, P. Olesen, Nucl. Phys. B 160, 380 (1979); H. B. Nielsen, M. Ninomiya, Nucl. Phys. B 156, 1 (1979); J. Amjorn, P. Olesen, Nucl. Phys. B 60, 1980 (.)

[84] G. Dosch, Phys. Lett. B 190, 555 (1987); G. Dosch, Y. Simonov, Phys. Lett. B 205, 339 (1988); Y. Simonov, Yad. Fiz. 48, 1381 (1988); Yad. Fiz. 50, 213 (1988).

[85] G. 'tHooft, Phys. Rev. Lett. 37, 8 (1976).

[86] J. Fingberg, J. Polonyi, Nucl. Phys. B 486, 315 (1997); V. Branchina and H. Mohrbach, J. Polonyi, Phys. Rev. D 60, 45006 (1999); Phys. Rev. D 60, 45007 (1999); M. Dufour Fournier, J. Polonyi, Phys. Rev. D 61, 065008 (2000).

[87] K. Huang, J. Polonyi, Int. J. of Mod. Phys. 6, 409 (1991).

[88] I. Nándori, J. Polonyi, K. Sailer, Phys. Rev. D 63, 045022 (2001).

[89] J. M. Kosterlitz, D. J. Thouless, JournalJ. Phys. CC61181973; J. M. Kosterlitz, JournalJ. Phys. CC710461974.

[90] J. V. Jose, L. P. Kadanoff, S. Kirkpatrick, D. R. Nelson, Phys. Rev. B 16, 1217 (1977).

[91] S. Coleman, Phys. Rev. D 11, 3424 (1975).

[92] S. Mandelstam, Phys. Rev. D 11, 3026 (1975).

[93] S. Samuel, Phys. Rev. D 18, 1916 (1978).

[94] J. Polonyi, The confinement-deconfinement mechanism, in Quark Gluon Plasma, World Scientific, 1990, R. Hwa, edt.

[95] N. D. Mermin, H. Wagner, Phys. Rev. Lett. 17, 1133 (1966); S. Coleman, Comm. Math. Phys. 31, 259 (1973).

[96] G. V. Gersdorff, C. Wetterich, Nonperturbative Renormalization Flow and Essential Scaling for the Kosterlitz-Thouless Transition, hep-th/0008114.

[97] C. Becchi, in Elementary Particles, Field Theory and Statistical Mechanics, Eds. M. Bonini, G. Marchesini, E. Onofri, Parma University 1993, hep-th/9607188; M. Bonini, M. D'Attanasio, G. Marchesini, Nucl. Phys. B 409, 1993 (441); Nucl. Phys. B 418, 1994 (81); Nucl. Phys. B 421, 1994 (429); Nucl. Phys. B 437, 1995 (163); M. Reuter, C. Wetterich, Nucl. Phys. B 417, 181 (1994); Nucl. Phys. B 427, 291 (1994); M. D'Attanasio, T. R. Morris, Phys. Lett. B 378, 213 (1996).

[98] D. F. Litim, J. M. Pawlowski, Phys. Lett. B 435, 181 (1998); F. Freire, D. F. Litim, J. M. Pawlowski,Phys. Lett. B 495, 256 (2000).

[99] T. R. Morris, Nucl. Phys. B 573, 97 (2000), J. High of Energy Phys. 0012, 012 (2000). 
[100] M. Simionato, Int. J. of Mod. Phys. 15, 2121 (2000); Int. J. of Mod. Phys. 15, 2153 (2000).

[101] J.Alexandre, J. Polonyi, K. Sailer, in preparation.

[102] K. Wilson, Rev. Mod. Phys. 55, 583 (1983).

[103] M. E. Fischer, in the Proceedings of Enrico Fermi International School of Physics, ed. M. S. Green (Academic Press, New York, 1971); M. P. Nightingale, Physica A 83, $561(1976)$.

[104] K. Wilson, M. E. Fischer, Phys. Rev. Lett. 28, 248 (1972); K. Wilson, Phys. Rev. D 7, 2911 (1973).

[105] P. C. Hohenberg, B. I. Halperin, Rev. Mod. Phys. 49, 435 (1977).

[106] G. 'tHooft, Proceedings of Colloquim on Renormalization of Yang-Mills Fields and Application to Particle Physics, ed. C. P. Korthes-Altes, (Marseilles, 1972); D. Gross, F. Wilcek, Phys. Rev. Lett. 30, 1343 (1973); H. D. Politzer, Phys. Rev. Lett. 30, 1346 (1973); S. Coleman, D. Gross, Phys. Rev. Lett. 31, 851 (1973).

[107] S. Weinberg, Physica A 96, 327 (1979); J. Gasser, H. Leutwyler, Ann. Phys. 158, 142 (1984); Nucl. Phys. B 250, 465 (1985); Phys. Rep. 87, 77 (1982); D. B. Kaplan, A. V. Manohar, Phys. Rev. Lett. 56, 2004 (1986).

[108] S. Weinberg, Phys. Lett. B 91, 51 (1980) I. J. R. Aitchison, Acta Phys. POlonica B 18, 191 (1987).

[109] N. Isgur, M. B. Wise, Phys. Lett. B 232, 113 (1989); Phys. Lett. B 237, 527 (1990). 